PNL-3831

Beginning-of-Life Data Report for , the Instrumented Fuel Assembly (IFA)-527

Prepared by D. D. Lanning

Pacific Northwest Laboratory

Operated by

Battelle Memorial Institute

Prepared for

U.S. Nuclear Regulatory

Commission 


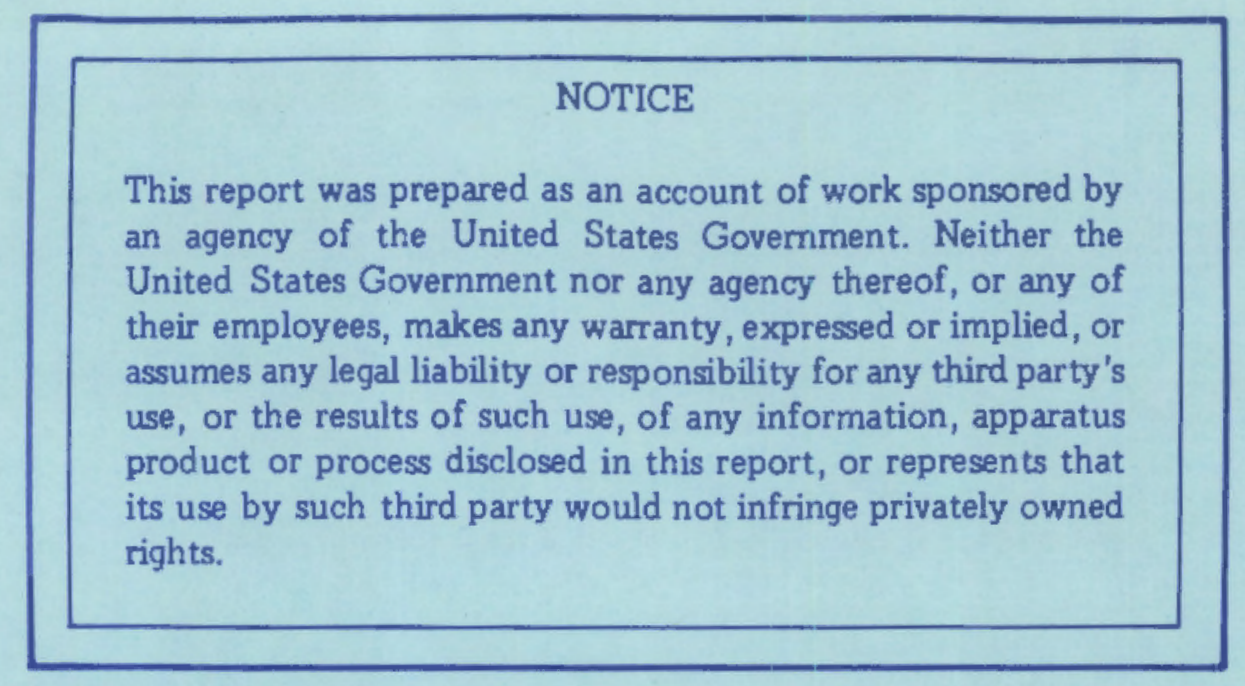

\author{
Available from \\ GPO Sales Program \\ Division of Technical Information and Document Control \\ U. S. Nuclear Regulatory Commission \\ Washington, D. C. 20555 \\ Printed copy price: $\$ 4.59$ \\ and
}

National Technical Information Service

Springfield, Virginia 22161 


\section{Beginning-of-Life Data Report for the Instrumented Fuel Assembly (IFA)-527}

Manuscript Completed: June 1981

Date Published: September 1981

Prepared by

D. D. Lanning

Pacific Northwest Laboratory

Richland, WA 99352

\section{Prepared for}

Division of Accident Evaluation

Office of Nuclear Regulatory Research

U.S. Nuclear Regulatory Commission

Washington, D.C. 20555

NRC FIN B2043 



\section{ACKNOWLEDGMENTS}

The editing of this report by S. K. Edler and helpful comments by M. E. Cunningham (PNL) and H. H. Scott (NRC - Reactor Safety Research) are gratefully acknowledged. 




\section{ABSTRACT}

This report presents beginning-of-life (BOL) data from the first four months of operation of the six-rod instrumented fuel assembly (IFA)-527 in the Halden Boiling Water Reactor (HBWR), Halden, Norway. This assembly is the last in a series of U.S. Nuclear Regulatory Commission (NRC)-sponsored tests to verify steady-state fue 1 performance computer codes. IFA-527 contains five identical rods with high-density stable fuel pellets and 0.23-mm diametral gaps and one rod with similar fuel pellets but with a 0.06-mm diametral gap. A11 six rods were xenon-filled to provide simulation of the effects of fission gas and to enhance the observable effects of fuel cracking and relocation on fue 1 temperatures. The assembly operated successfully from July 1, 1980, to August 15, 1980; and then the reactor was shut down until September 10, 1980. Sometime during the shutdown, four of the six rods suffered pressure boundary failure. The decision was made to restart the reactor to collect operating data with failed rods. This report presents both pre- and postfailure data for IFA-527. 


\section{SUMMARY AND CONCLUSIONS}

The NRC/PNL Halden instrumented fuel assembly (IFA)-527 carries six heavily instrumented test fuel rods that are each filled with xenon gas rather than the standard helium gas. The purpose of these xenon-filled rods was to provide test data related to the worst-case effects of fission gas on fuel temperatures and to enhance the observable effects of fuel cracking and relocation on fuel temperatures. The purpose of having several rods of identical design was to assess the variability of relocation and its consequences. Power was held very low (18.0 $\mathrm{kW} / \mathrm{m}$ lifetime peak power) to maintain fuel temperatures within the range of commercial reactor experience.

The startup, calibration, and first operating cycle for this assembly were highly successful. Temperatures from the nominal-and small-gap rods provide a bracket for the expected temperatures for fission gas-saturated rods; fuel relocation in the peak-power sections of the rods became evident in the first month of operation. Enhanced relocation, even at startup, was evident in at least some of the nominal-gap $(230-\mu m)$ rods by the trend of their elongation versus power. Finally, the behavior of the five identical nominal-gap rods was very similar as judged from temperature, elongation, and gas pressure data.

Unfortunately, four of the rods failed during the long shutdown between August and September 1980. These failures manifested themselves in abnormally high pressure readings and (simultaneous) abnormally low fuel temperatures. In spite of the apparent pressure boundary failure and water inleakage, no fission product leakage was detected; and the thermocouples appeared to continue working properly. The latter was confirmed by analysis of transient fuel temperature responses. When postirradiation examination (PIE) is conducted, these rods will provide valuable insight into the nature of incipient failure and the possibie condition and existence of undetected water-logged commercial reactor rods. 



\section{CONTENTS}

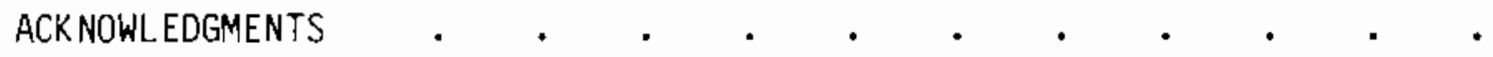

ABSTRACT .

SUMMARY AND CONCLUSIONS

INTRODUCTION

ASSEMBLY DESCRIPTION AND INSTRUMENTATID

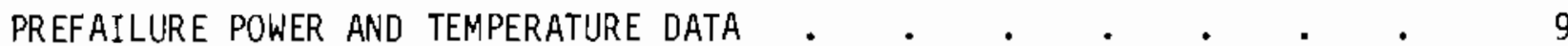

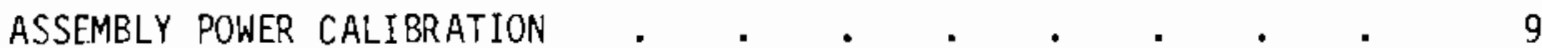

FUEL TEMPERATURE AND POWER DATA DURING STEADY-STATE OPERATION $\quad \cdot \quad 12$

Temperature (Resistance) Increases on Fjrst Startup . $\quad 12$

Comparative Performance of Rods 1 Through 5 . $\quad . \quad$. $\quad 19$

Temperature Behavior from July 5 to August $15,1980 \quad$ • $\quad 21$

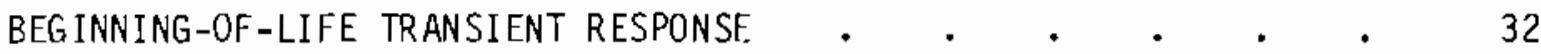

SUMMARY OF PREFAILURE THERMAL PERFDRMANCE • • . • . • • 36

PREFAILURE CLADDING ELONGATION AND GAS PRESSURE DATA $\quad$ • $\quad$ • $\quad$ • 37

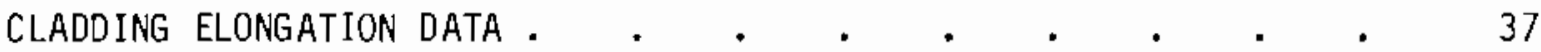

Initial Ramp Data $. \quad . \quad . \quad . \quad . \quad . \quad . \quad . \quad 37$

Behavior During First Operating Period . . . . . . 45

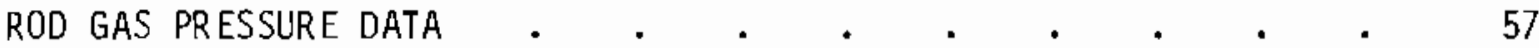

Initial Ramp Data . . . . . . . . . . 57

Behavior During First Operating Period . • . . . 59

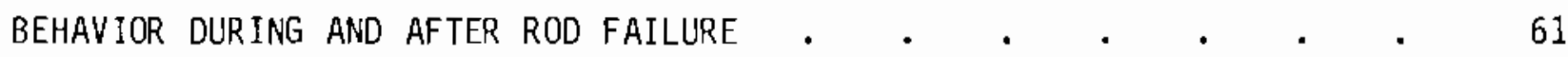

TRANSIENT TEMPERATURE CONFIRMATION OF IFA-527 POSTFAILURE

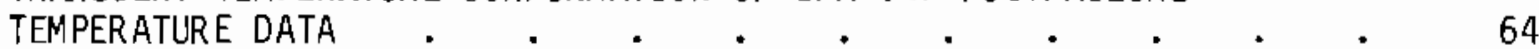

PRE- AND POSTFAILURE COMPARISON OF GAS PRESSURE AND CLADDING

ELONGATION BEHAVIOR .

REFERENCES $\quad \cdot \quad \cdot \quad \cdot \quad \cdot \quad \cdot \quad \cdot \quad \cdot \quad \cdot \quad \cdot \quad \cdot \quad \cdot \quad \cdot \quad 79$ 



\section{FIGURES}

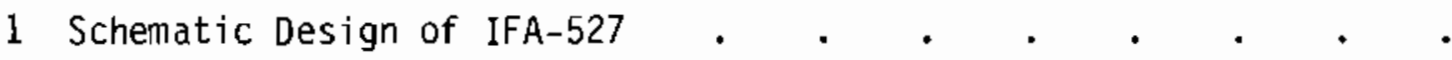

2 Arrangement of Temperature Sensors, Neutron Detectors, and Fue? Relative to Reference Axial Thermal Flux Profile for Instrumented Fuel Assembly (IFA)-527 . . . . . . . . . .

3 Instrumented Fuel Assembly (IFA)-527 Arrangement in the Halden Reactor

4 Subcooled Calibration Data Plots Showing Subcooling Power (QSC), Neutron Detector Average ( $\overline{\mathrm{ND}}$ ), and Channel Coolant Temperature Rise (DTCH) Plotted Against Coolant-Moderator Temperature Difference (DTM)

5 Upper and Lower Thermocouple Readings and Associated Linear Heat Ratings for Rod 1 of IFA-527; First Rise to Power . .

6 Upper and Lower Thermocouple Readings and Associated Linear Heat Ratings for Rod 2 of IFA-527; First Rise to Power . .

7 Upper and Lower Thermocouple Readings and Associated Linear Heat Ratings for Rod 3 of IFA-527; First Rise to Power .

8 Upper and Lower Thermocouple Readings and Associated Linear Heat Ratings for Rod 4 of IFA-527; First Rise to Power . . . 16

9 Upper and Lower Themocouple Readings and Associated Linear Heat Ratings for Rod 5 of IFA-527; First Rise to Power . . . 17

10 Upper and Lower Thermocouple Readings and Associated Linear Heat Ratings for Rod 6 of IFA-527; First Rise to Power . . . 18

11 Temperature Versus Power for Lower Thermocouple of Rod 1 Through the First 12 Days of Irradiation . . . . . . .

12 Temperature Increments Experienced During First Full-Power Hold Period Versus Initial Temperature at the Start of the Hold . .

13 Stabilized Temperature Versus Power Data for Rods 1 Through 5 (230- 2 m gap rods) Relative to a Calculated Uncertainty Band

14 Fuel Centerline Temperatures Versus Time for Rod 1 for the First Operating Period (July 1 to August 15, 1980) . . . .

15 Fuel Centerline Temperatures Versus Time for Rod 2 for the First Operating Period (July 1 to August 15, 1980) 
16 Fuel Centeriine Temperatures Versus Time for Rod 3 for the First Operating Period (July 1 to August 15, 1980) . . . . .

17 Fuel Centerline Temperatures Versus Time for Rod 4 for the First Operating Period (July 1 to August 15, 1980) . . . . .

18 Fuel Centerline Temperatures Versus Time for Rod 5 for the First Operating Period (July 1 to August 15, 1980) . . . .

19 Fuel Centerline Temperatures Versus Time for Rod 6 for the First Operating Period (July 1 to August 15, 1980) . . . .

20 Total Assembly Power Versus Time for the First Operating Period (July 1 to August 15, 1980)

21 Stabilized Upper/Lower Fuel Centerline Temperatures Versus Local Linear Heat Rating for Rod 1 on July 8 and $9,1980 . .$.

22 Stabilized Upper/Lower Fuel Centerline Temperatures Versus Local Linear Heat Rating for Rod 1 on August 12, 1980 . . . . . 30

23 Lower Thermocouple Readings for Rod 1 Versus Linear Heat Rating on July 8 and August 12, 1980 . . . . . . . . .

24 Normalized Power and Fuel Centerline Thermocouple Data for Linear Power Decrease

25 Temperature Versus Power for Rod 1 (Lower) from July 1-12, 1980 , Showing MWRAM Best-Estimate Input Conditions for Run 174

Simulation.

26 Measured and MWRAM Calculated Fuel Centerline Temperature Response for Run 174 for Rod $1 \quad$. $1 . . . \quad$. .

27 Fuel Rod Elongation and Average Linear heat Rating for Rod 1 During the First Rise to Power.

28 Fue? Rod Elongation and Average Linear Heat Rating for Rod 2 During the First Rise to Power . . . . . . . .

29 Fuel Rod Elongation and Average Linear Heat Rating for Rod 3 During the First Rise to Power . . . . . . .

30 Fuel Rod Elongation and Average Linear Heat Rating for Rod 4 During the First Rise to Power . . . . . . . .

31 Fuel Rod Elongation and Average Linear Heat Rating for Rod 5 During the First Rise to Power . . . . . . .

32 Fuel Rod Elongation and Average Linear Heat Rating for Rod 6 During the First Rise to Power. 
33 Coolant Channel Temperature Rise During the First Rise to Power .

34 Coolant Channel Temperature Rise During the First Operating Period

35 Rod Elongation Versus Average Linear Heat Rating During Second Rise to Power for Rods 1 and $6 . . . .$. . . . .

36 Rod Elongation Versus Average Linear Heat Rating for Rods 1 Through 5 on July $9,1980 . . \quad . \quad . \quad . \quad . \quad . \quad .48$

37 Rod Elongation Versus Average Linear Heat Rating for Rods 1

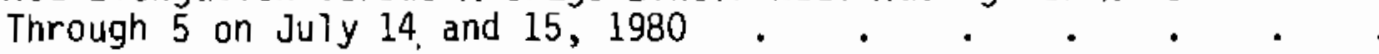

38 Rod Elongation Versus Average Linear Heat Rating for Rods 1

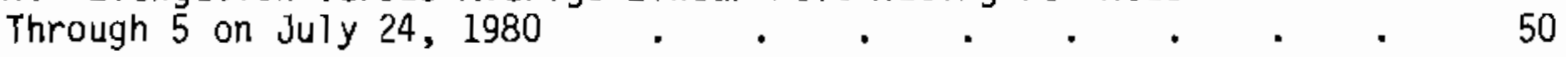

39 Elongation Versus Time During the First Operating Period for Rod 1

40 Elongation Versus Time During the First Operating Period for Rod 2

41 Elongation Versus Time During the First Operating Period for Rod $3 \quad 53$

42 Elongation Versus Time During the First Operating Period for Rod $4 \quad 54$

43 Elongation Versus Time During the First Operating Period for Rod $5 \quad 55$

44 Elongation Versus Time During the First Operating Period for Rod $6 \quad 56$

45 Relative Gas Pressure for Rods 1 Through 5 on First Rise to Power . $\quad 58$

46 Fuel Rod Gas Pressure for Rods 1 Through 5 During the First

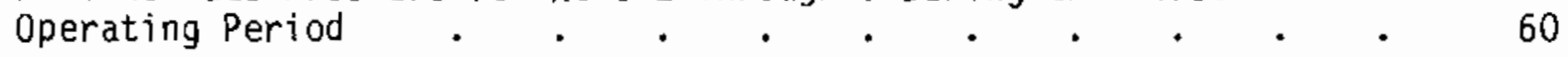

47 Gas Pressure Behavior of Rods 1 Through 5 During the September 10-12, 1980, Startup. . . . . . . . 62

48 Lower Fuel Centerline Temperatures for Rods 1, 2, and 3 During the September 10-12, 1980, Startup . . . . . . .

49 Measured and Calculated Centeriine Temperatures for Rods 1 and 3 (failed and unfailed) in September 1980 . . . . . . .

50 Transient Temperature Response of Representative (1ower position) Thermocouples for Run 187 . . . . . . . 66

51 Pre- and Postfailure Gas Pressure Behavior for Rod 1 . . . 68

52 Pre- and Postfailure Gas Pressure Behavior for Rod 2 . . $\quad . \quad 69$ 
53 Pre- and Postfailure Gas Pressure Behavior for Rod 3 . . . 70

54 Pre- and Postfailure Gas Pressure Behavior for Rod $4 \quad$ • . . 71

55 Pre- and Postfailure Gas Pressure Behavior for Rod 5 . . . $\quad 72$

56 Pre- and Postfailure Elongation Behavior for Rod 1 . . . . 73

57 Pre- and Postfailure Elongation Behavior for Rod 2 . $\quad$. . 74

58 Pre- and Postfailure Elongation Behavior for Rod 3 . . . . 75

59 Pre- and Postfailure Elongation Behavior for Rod 4 . . . . . 76

60 Pre- and Postfailure Elongation Behavior for Rod 5 . . . . . 77

61 Pre- and Postfailure Elongation Behavior for Rod 6 . . . . . . 78 


\section{TABLES}

1 General Design Characteristics for NRC/PNL Instrumented Fuel Assemblies.

2 Experimental Matrix for Instrumented Fuel Assemblies-431, $-432,-513$, and -527 .

3 Measured and Calculated Temperature Slopes for Rod 1 (Lower), Run $174 \quad$. $\quad . \quad$. $\quad . \quad$. $\quad . \quad$.

4 Measured and Caiculated Temperature Slopes for Rod 6, Run 174

5 Estimated Void Volume Distribution for Rods 1 Through 5 of Instrumented Fuel Assembly (IFA)-527

6 Measured and Calculated Normalized Temperature Slopes from Run 187 



\section{INTRODUCTION}

This report presents data from the first four months of operation of the six-rod instrumented fuel assembly (IFA)-527, which was sponsored by the U.S. Nuclear Regulatory Commission (NRC) and irradiated in the Halden Boiling Water Reactor (HBWR) in Norway. It is the final assembly in a series of NRCsponsored tests to verify steady-state fuel performance computer codes. Al] the rods in the series are of the basic BWR- 6 design with variations in gap size, fuel pellet type, fill gas composition, and fill gas pressure.

The first two tests in this Pacific Northwest Laboratory (PNL) ${ }^{(a)}$ series were IFA-431 and -432. These identical assemblies each contained the same variations of gap sizes and fuel peliet types, but they were run at different power jevels and to different burnups. The third assembly, IFA-513, was jointly sponsored by NRC and the Halden Reactor Project. It has six identical rods with high-density stable $\mathrm{UO}_{2}$ fuel and a nominal pellet-cladding diametral gap size of $0.23 \mathrm{~mm}$ but with variations in fill gas composition and pressure.

The fourth and final assembly in the series--IFA-527--contains five identical rods with high-density stable fuel pellets and 0.23-mm diametral gaps; the sixth rod has a much smaller gap--0.06 mm. All six rods were filled with xenon gas to enhance the observable thermal effects of fuel pellet cracking and relocation while minimizing the effect of fission gas released.

Measurements were made in IFA-527 and earlier tests on a continuous basis, providing a record of their variation with both power and burnup; measurements included:

- fuel centerline temperature and power (steady-state and transient)

- total fuel rod elongation

- fuel rod internar gas pressure.

A major occurrence in IFA-527 was the pressure boundary failure in four or the six rods after two months in the reactor. Most of the instrumentation in

(a) Operated for the U.S. Department of Energy (DOE) by Battelle Memorial Institute. 
both failed and unfailed rods survived the pressure boundary failure. The decision was made to restart the reactor with the failed rods operating to collect such rare comparative data; in addition, no detectable fission products had escaped from the assembly. The behavior of the rods before and after failure is presented in this report; minimal analyses required to put IFA-527 in perspective relative to IFA-513, -432 , and -431 are also discussed. 


\section{ASSEMBLY DESCRIPTION AND INSTRUMENTATION}

IFA-527 (see Figure 1) was designed with six heavily instrumented test fuel rods that were to be filled with xenon gas and contain $95 \%$ theoretical density (TD), flat-ended $\mathrm{UO}_{2}$ pellets enriched to $10 \%$ uranium-235. Each rod was instrumented with a pressure transducer, an elongation sensor, and fuel centerline thermocouples in both ends. Fabrication details and instrumentation calibration for both the rods and the final assembly are contained in the precharacterization report (Cunningham 1981). The assembly was designed with the following purposes in mind:

- to include, within the publicly documented NRC Halden Test Series, rods with pure xenon fill gas, thus bracketing the worst-case estimates of fuel temperature dependence on power

- to study fuel cracking and relocation effects upon fuel temperatures without the added complication of fill gas themal conductivity degradation due to fission gas release

- to assess not only the speed but also the variability of fuel cracking and relocation effects by including several rods of identical design that are operated identically

- to possibly confirm burnup enhancement of fission gas release by operating these rods to high burnup at constant power and (because of xenon fill gas) constant temperature.

Tables 1 and 2 contrast design features and instrumentation of IFA-527 with those of previous assemblies. (a) IFA-527 is very similar to IFA-513 in design; one major difference is the inclusion of a small-gap rod. 0ther design and operating differences are as follows:

- IFA-527 operated at lower powers than IFA-513 (18 kW/m peak power as opposed to $42 \mathrm{~kW} / \mathrm{m})$.

(a) Design data for IFA-431, -432 , and -513 were drawn from their respective precharacterization reports (Bradley et al. 1979; Hann et al. 1977). 


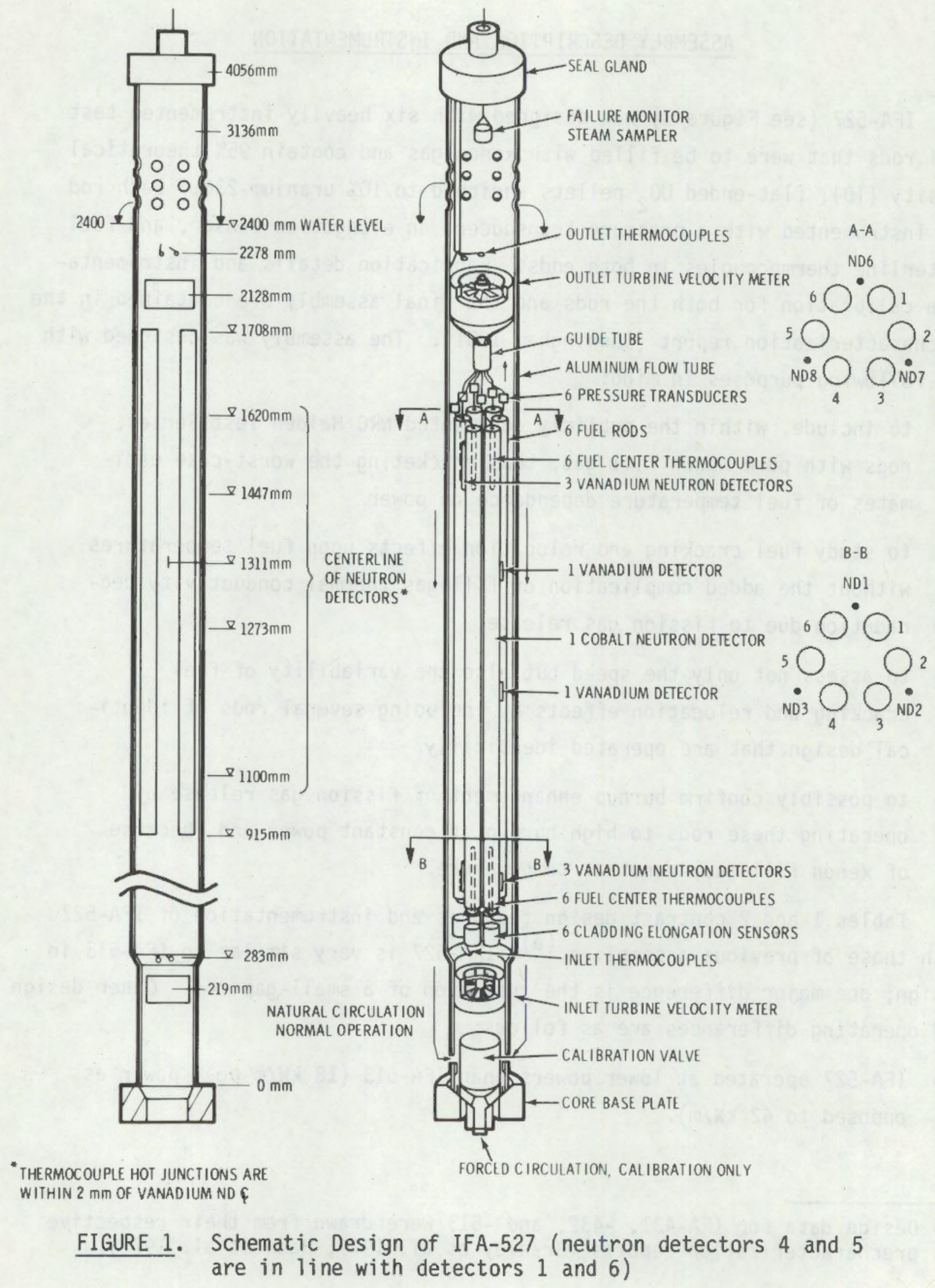


TABLE 1. General Design Characteristics for NRC/PNL Instrumented Fuel Assemblies

\begin{tabular}{|c|c|c|}
\hline Characteristic & IFA-513 and -527 & IFA-431 and -432 \\
\hline Number of rods & 6 & 6 \\
\hline Cladding: & & \\
\hline Material & Zircaloy-2 & Zircaloy-2 \\
\hline Inside Diameter, mm & 10.9 & 10.9 \\
\hline Outside Diameter, mm & 12.8 & 12.8 \\
\hline Tube Length, mm & 824 & 610 \\
\hline Fuel Pellets: & & \\
\hline Material & $\mathrm{UO}_{2}$ & $\mathrm{UO}_{2}$ \\
\hline Density, \%TD & 95,92 & $95^{2}$ \\
\hline Enrichment, \% $235 \mathrm{U}$ & 9.9 & 10.0 \\
\hline Inside Diameter, mm & 1.75 & 1.75 \\
\hline Outside Diameter, $\mathrm{mm}$ & 10.67 & 10.67 (nominal) \\
\hline Pellet Length, min & 12.7 & 12.7 \\
\hline Pellet Column Length, mm & 780 (nominal) & 570 (nominal) \\
\hline (each end), mm & 7 & 7 \\
\hline Fuel Rod Plenum Length, mm & 29 & 19 \\
\hline
\end{tabular}

- While all previous assemblies had peak power at or near the top end, IFA-527 was located in the top half of the reactor and had its peakpower location at the bottom end. The axial location of the assembly relative to the reactor flux shape is shown in Figure 2.

- The assembly was located at the extreme edge of the reactor core to assure the low powers needed to hold fuel temperatures within the range of cormercial reactor experience (see Figure 3 ). This extreme edge position suffers a significant thermal neutron flux tilt across the assembly channel (edge-to-edge differences are in excess of 15\%). This was confirmed by both vanadium neutron detector data and fuel centerline temperature data.

- Single-phase rather than two-phase flow was experienced during the assembly power calibration. 
TABLE 2. Experimental Matrix for Instrumented Fuel Assemblies-431, $-432,-513$, and -527

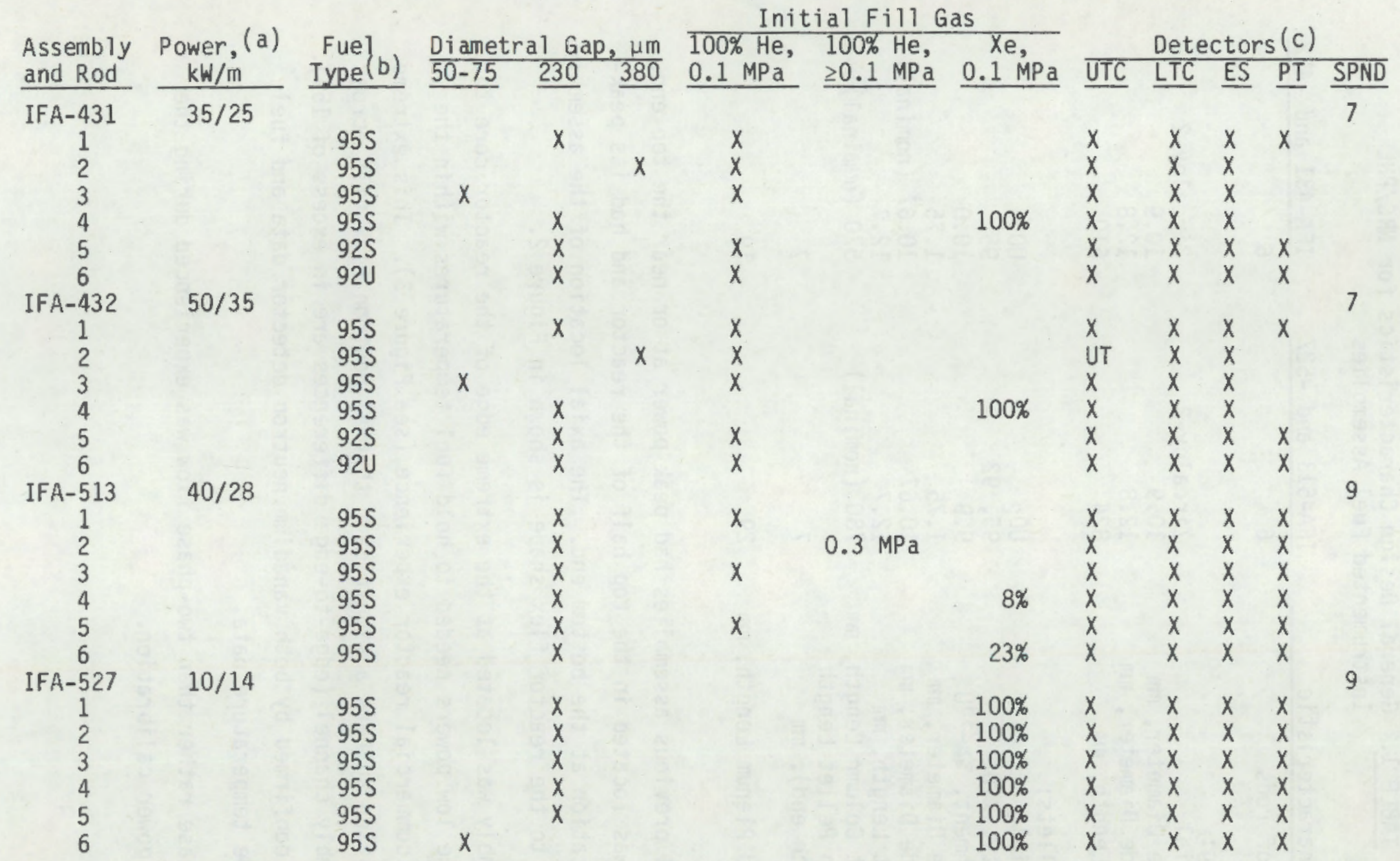

(a) Linear power is given for upper and lower thermocouple positions, respectively.

(b) Three fuel types are used, all enriched to $10 \% 235 \mathrm{U}$ : $95 \mathrm{~S}=95 \%$ To, Stable; $92 \mathrm{~S}=92 \%$ TD, Stable; $92 \mathrm{U}=92 \% \mathrm{TO}$, Unstable.

(c) UTC = Upper Thermocouple; LTC = Lower Thermocouple; ES = Elongation Sensor; PT = Pressure Transducer; SPND = Self-Powered Neutron Detector; UT = Ultrasonic Thermometer. 


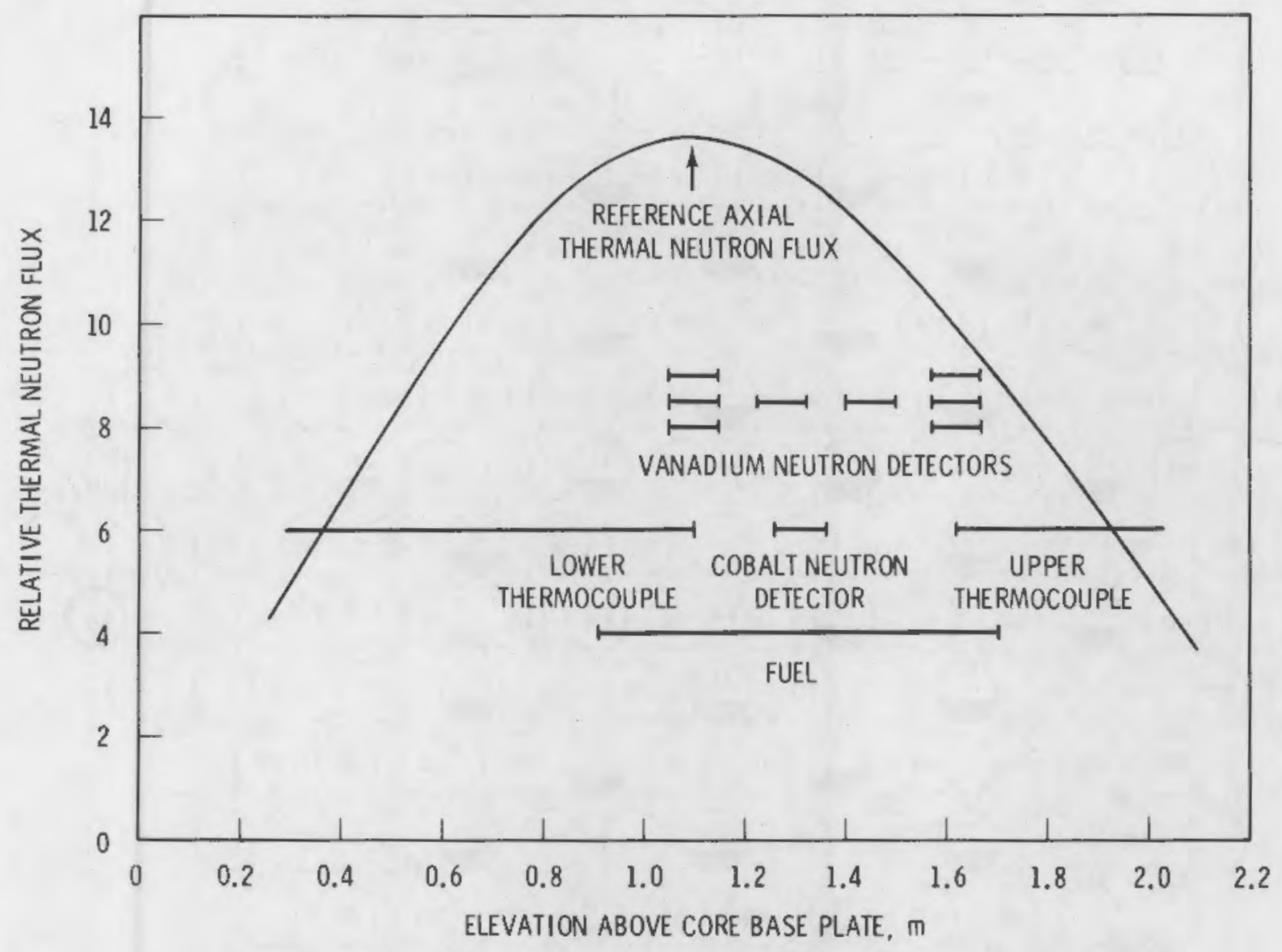

FIGURE 2. Arrangement of Temperature Sensors, Neutron Detectors, and Fuel Relative to Reference Axial Thermal Flux Profile for Instrumented Fuel Assembly (IFA)-527

Except for the above differences, the assembly design, instrumentation, and operation of IFA-527 were very similar to those of IFA-513. Both assemblies had eight vanadium neutron detectors: three at both the upper and lower planes of fuel temperature measurement and two at equal intervals between those planes so that both the flux tilt at the thermocouple planes and the axial distribution of neutron flux might be confirmed.

In addition, both assemblies have a fast-reacting cobalt neutron detector at the axial and radial center to provide the relative variation of flux and hence power level during fast transients. This is especially useful in analyzing fuel thermocouple response to rapid linear and "step" power changes, both of which have been shown useful in confirming steady-state data trends and thermocouple reliability (Lanning et al. 1979; Lanning 1979). 


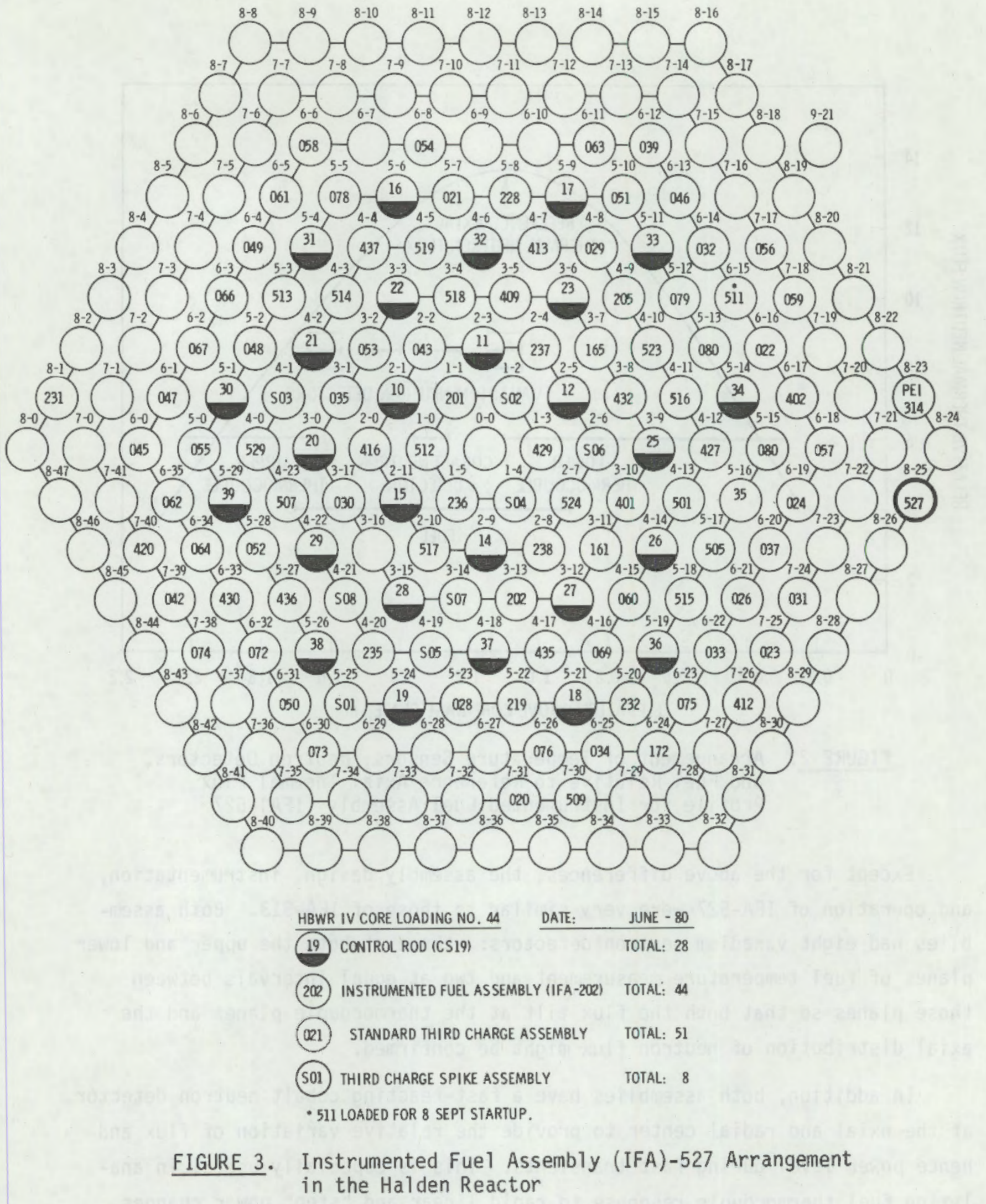


PREFAILURE POWER AND TEMPERATURE DATA (July 1 to August 15,1980 )

This section deals with the power and fuel centerline temperature data prior to the fuel rod failures that occurred in September 1980. The assembly power calibration and the calculation of local linear heat rating from the neutron detector readings are described to emphasize our confidence in those estimates. The dependence of the fuel centerline temperature on these estimated powers is then described. It will be shown that the performance variation among the five rods with $230-\mu m$ fabricated gaps is quite small.

\section{ASSEMBLY POWER CALIBRATION}

As noted in Figure 1, IFA-527 was equipped with inlet and outlet flowmeters and with two pairs of inlet/outlet differential thermocouples. This instrumentation is used to estimate the total channe 1 power from the coolant mass flow and at temperature rise; this is during special "subcooling ramps" early in the life of an assembly when the power is held constant and the degree of subcooling (i.e., temperature below saturation temperature) is varied for the inlet coolant.

Normally, subcooling ramps would be performed with various degrees of boiling permitted to provide good coolant mixing. The boiling power would be estimated from inlet/outlet flows and temperatures using equations relating flow and quality. Channel power would be estimated by extrapolation to zero boiling power.

The low powers experienced by IFA-527 on startup permitted a different calibration procedure. Boiling was totally suppressed, and the channel power was measured directly from the inlet flow and the coolant temperature rise along the channel. An "uncorrected" calibration factor, KG, was then found by dividing the apparent assembly power, QSC, by an appropriate average of the neutron detector readings, $\overline{\mathrm{ND}}$. (a)

(a) For IFA-527, the average, $\overline{\mathrm{ND}}$, that Halden recommends is $\overline{\mathrm{ND}}=\frac{1}{2}\left[\frac{\mathrm{ND1}+\mathrm{ND2}+\mathrm{ND3}}{3 \mathrm{ND1}}+\frac{\mathrm{ND6}+\mathrm{ND7}+\mathrm{ND8}}{3 \mathrm{ND6}}\right]\left(\frac{\mathrm{ND1}+\mathrm{ND4}+\mathrm{ND5}+\mathrm{ND6}}{4}\right)$ where $\mathrm{ND}_{j}=$ current reading of $i^{\text {th }}$ detector (nanoamps). 
Figures $4 \mathrm{a}$ and $4 \mathrm{~b}$ show the pertinent data from subcooling ramps 1 and 3. (a) All data are plotted versus the mean moderator-coolant temperature difference, DTM. The sharp bend in the plot of channel coolant temperature rise (DTCH) versus DTM marks the level of DTM (the level of subcooling) that successfully suppresses boiling. Values of QSC to the right of this critical level are plotted versus DTM, and the results are extrapolated back to nearzero DTM. The actual extrapolated values of QSC and $\overline{N D}$ used to calculate KG are those at DTM $=2^{\circ} \mathrm{C}$ since these correspond to a position of zero net heat exchange with the moderator. These values are noted with the subscript "N" in Figures $4 \mathrm{a}$ and $4 \mathrm{~b}$.

The channel power must be corrected by subtracting the gamma heating of the component. Halden has estimated this gamma heating from both internal and external sources to be $3.9 \%$ of the assembly power. The average net calibration factor for the assembly is thus:

$$
K G_{f}=\left(\frac{1.896+1.883}{2}\right)(1-0.039)=1.816 \mathrm{~kW} / \mathrm{nA}
$$

Rod powers are found from the assembly power by correcting the neutron detector readings back to the centerlines of the rods at the four axial locations of neutron flux measurement and then integrating the flux distribution over the rod length for each of the six rods. This gives the partition of powers among the rods. Local linear heat generation rates are found by multiplying the local neutron detector reading (corrected to the rod centerline position) times a factor that is the ratio of the rod power to the integrated detector reading for that rod. That is,

\section{local linear heat rating $=($ corrected detector reading) $\star$ factor}

$$
\text { where factor }=\frac{\text { rod power }}{\int_{0}^{L}{ }_{N D}^{(\ell)} d}
$$

(a) The second calibration ramp was invalidated by instrument failure (outlet turbine). 
a) Ramp 1

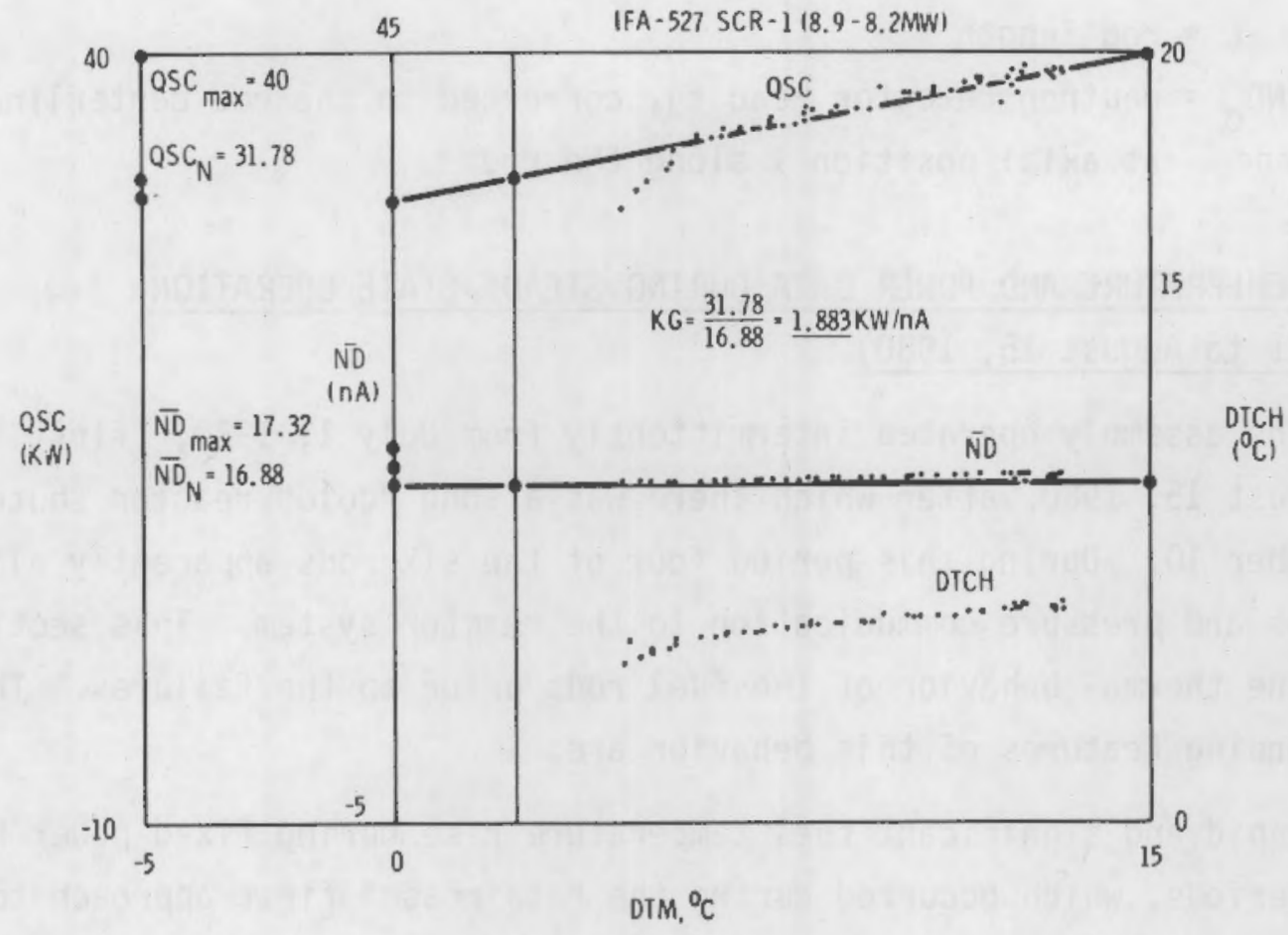

b) Ramp 3

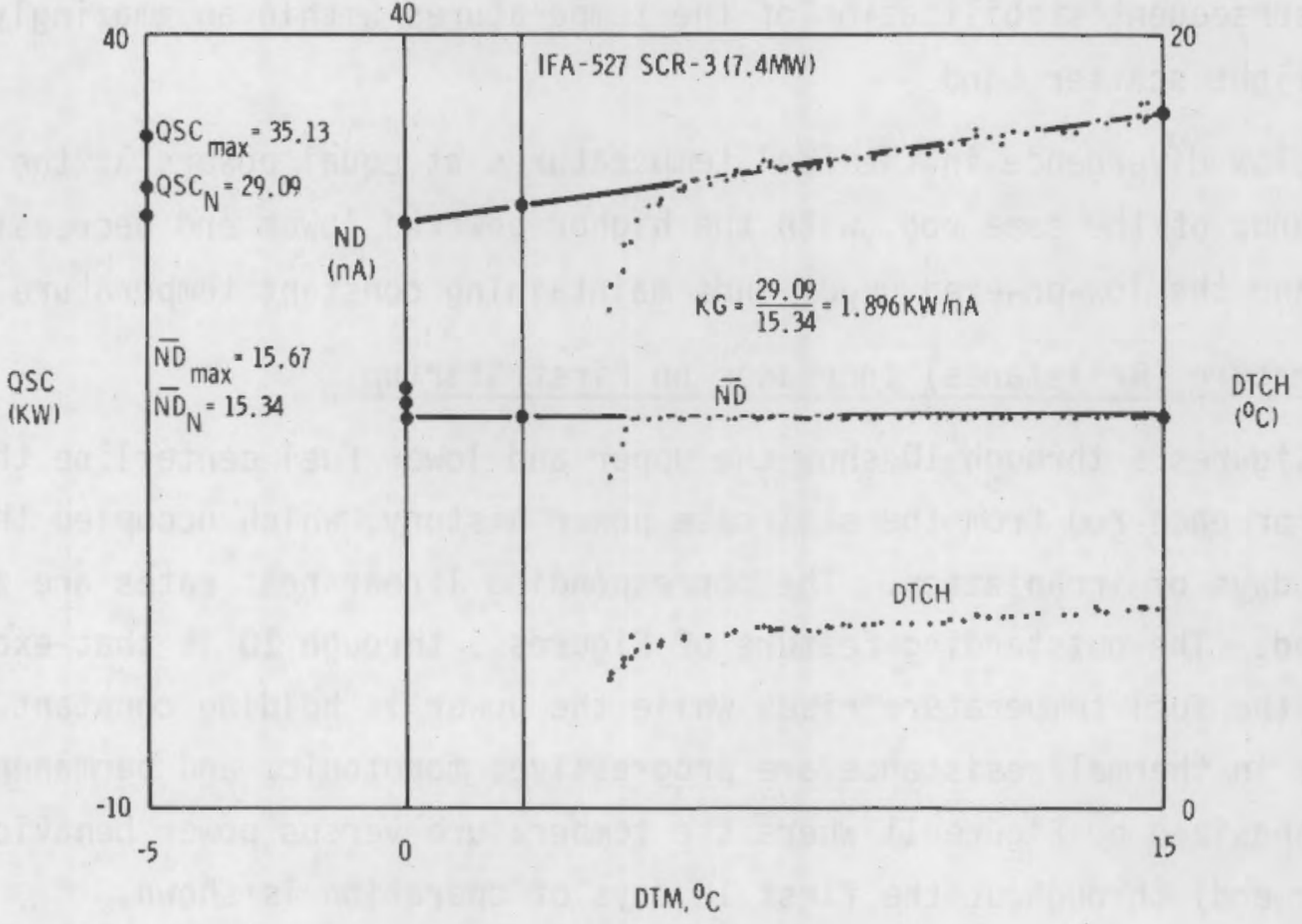

FIGURE 4. Subcooled Calibration Data Plots Showing Subcooling Power (QSC), Neutron Detector Average ( $\overline{N D})$, and Channel Coolant Temperature Rise (DTCH) Plotted Against Coolant-Moderator Temperature Difference (DTM) 
where

$$
\begin{aligned}
L= & \text { rod length } \\
N D_{C}= & \text { neutron detector reading, corrected to the rod centerline } \\
& \text { at axial position } \ell \text { along the rod. }
\end{aligned}
$$

FUEL TEMPERATURE AND POWER DATA DURING STEADY-STATE OPERATION

(July 1 to August 15,1980 )

The assembly operated intermittently from July 1, 1980, (first startup) to August 15, 1980, after which there was a long "cold" reactor shutdown until September 10. During this period four of the six rods apparently allowed water ingress and pressure communication to the reactor system. This section deals with the thermal behavior of the fuel rods prior to the failures. The three outstanding features of this behavior are:

- rapid and significant fuel temperature rise during fixed-power hold periods, which occurred during the "staircase" first approach to power

- subsequent stabilization of the temperatures within an amazingly tight scatter band

- slow divergence in the fuel temperatures at equal powers at the two ends of the same rod, with the higher powered lower end decreasing and the low-powered upper ends maintaining constant temperature.

Temperature (Resistance) Increases on First Startup

Figures 5 through 10 show the upper and lower fuel centerline thermocouple data for each rod from the staircase power history, which occupied the first three days of irradiation. The corresponding linear heat rates are also plotted. The outstanding feature of Figures 5 through 10 is that except for rod 6 the fuel temperature rises while the power is holding constant. These shifts in thermal resistance are progressive, monotonic, and permanent, which is emphasized by figure 11 where the temperature versus power behavior of rod 1 (lower end) throughout the first 12 days of operation is shown. 


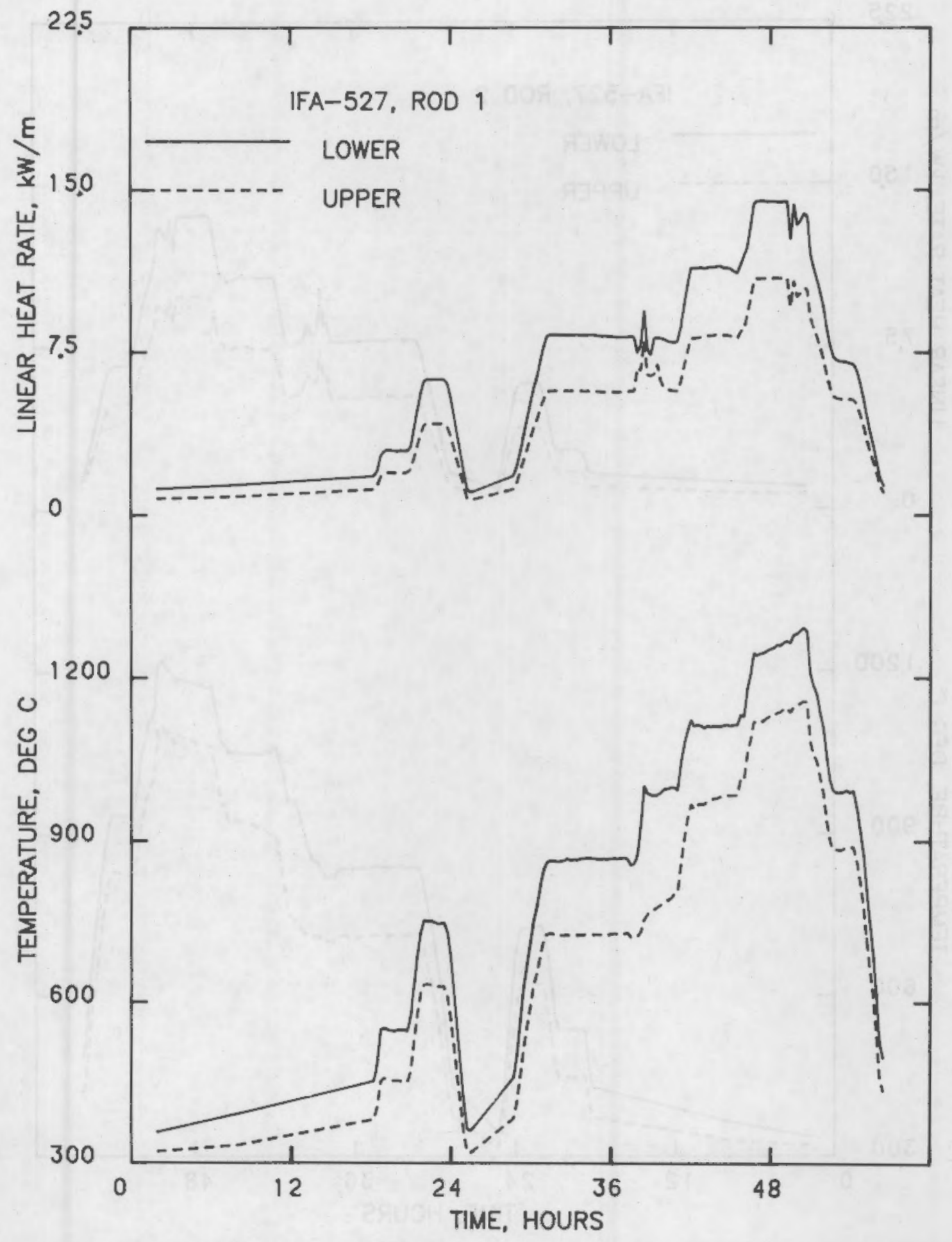

FIGURE 5. Upper and Lower Thermocouple Readings and Associated Linear Heat Ratings for Rod 1 of IFA-527; First Rise to Power 


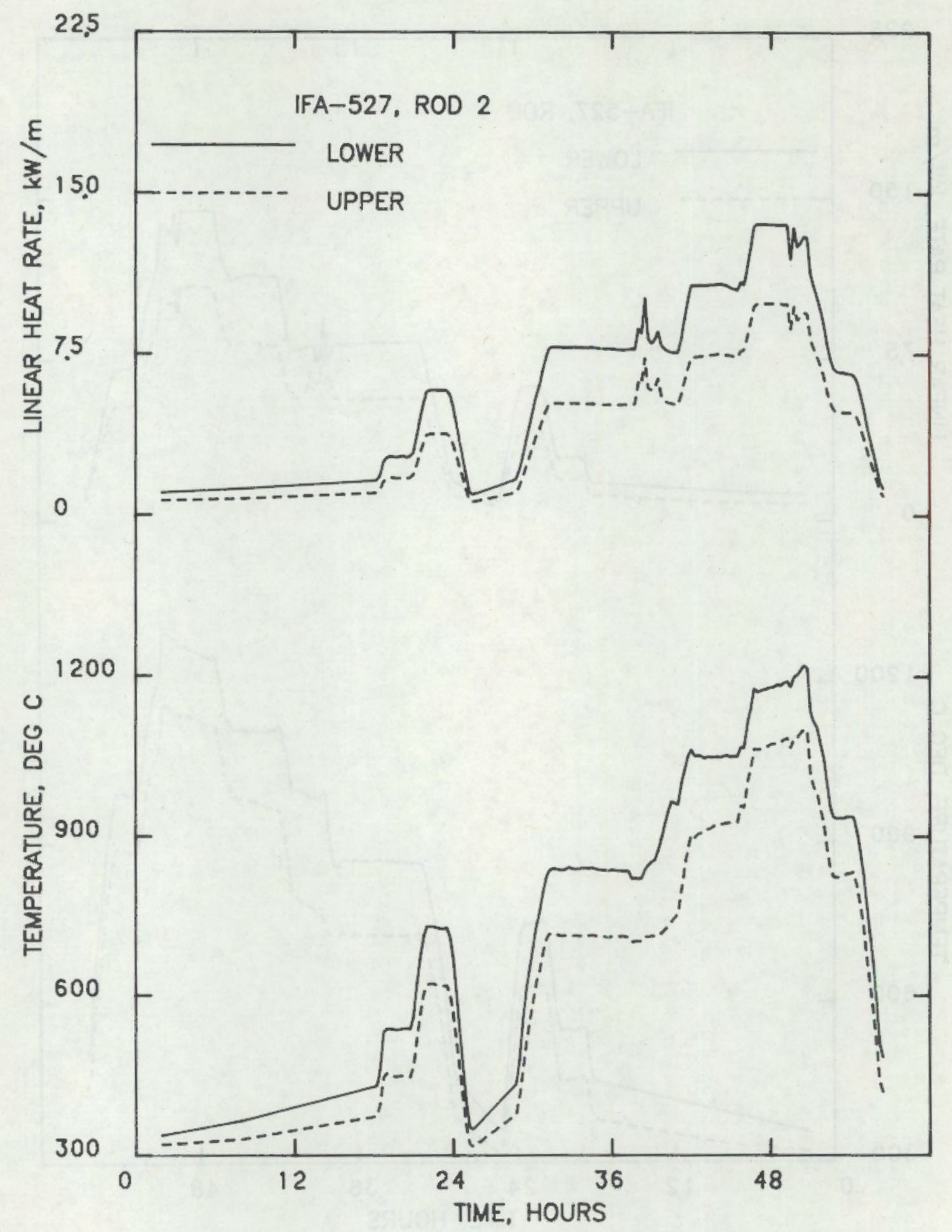

FIGURE 6. Upper and Lower Thermocouple Readings and Associated Linear Heat Ratings for Rod 2 of IFA-527; First Rise to Power 


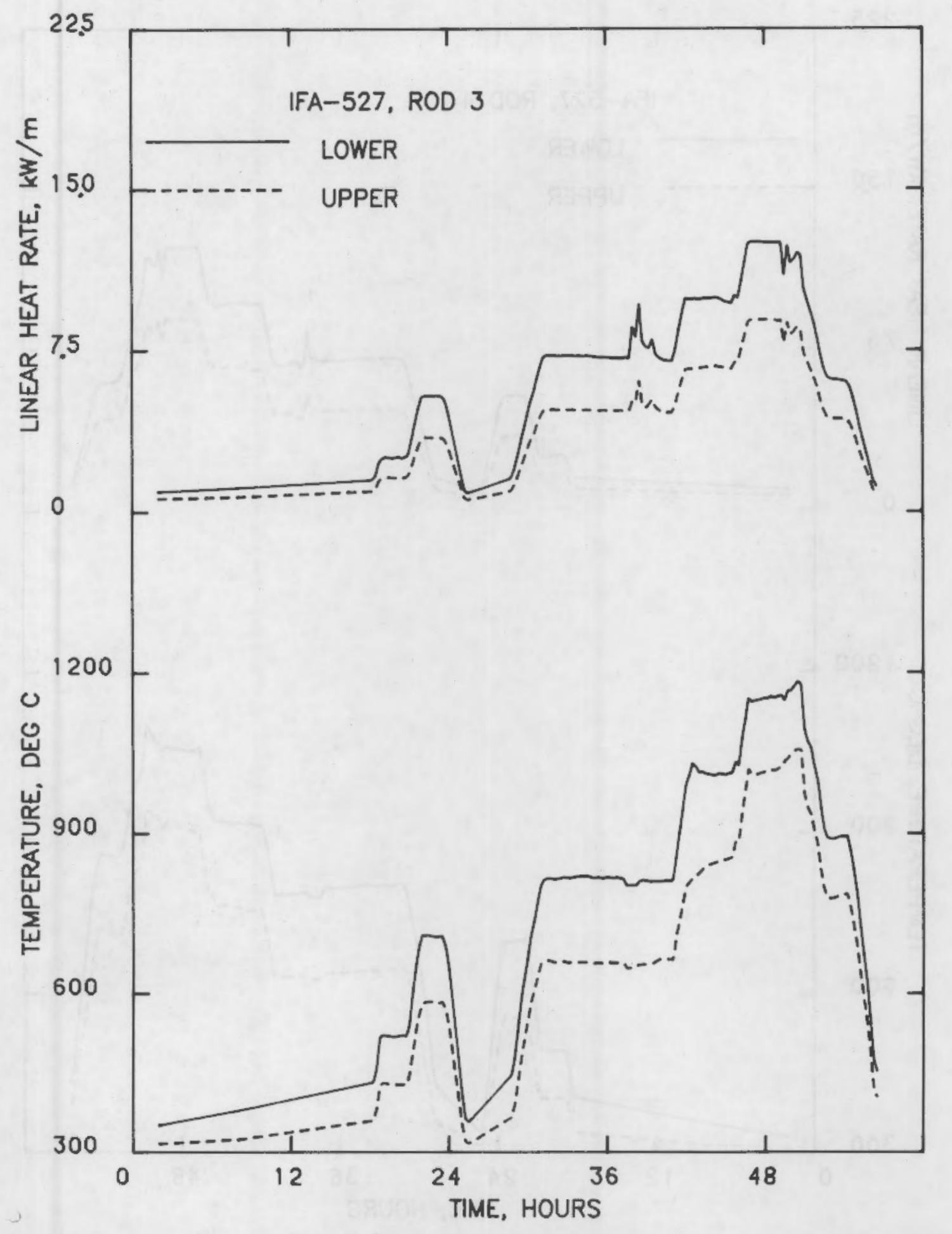

FIGURE 7. Upper and Lower Thermocouple Readings and Associated Linear Heat Ratings for Rod 3 of IFA-527; First Rise to Power 


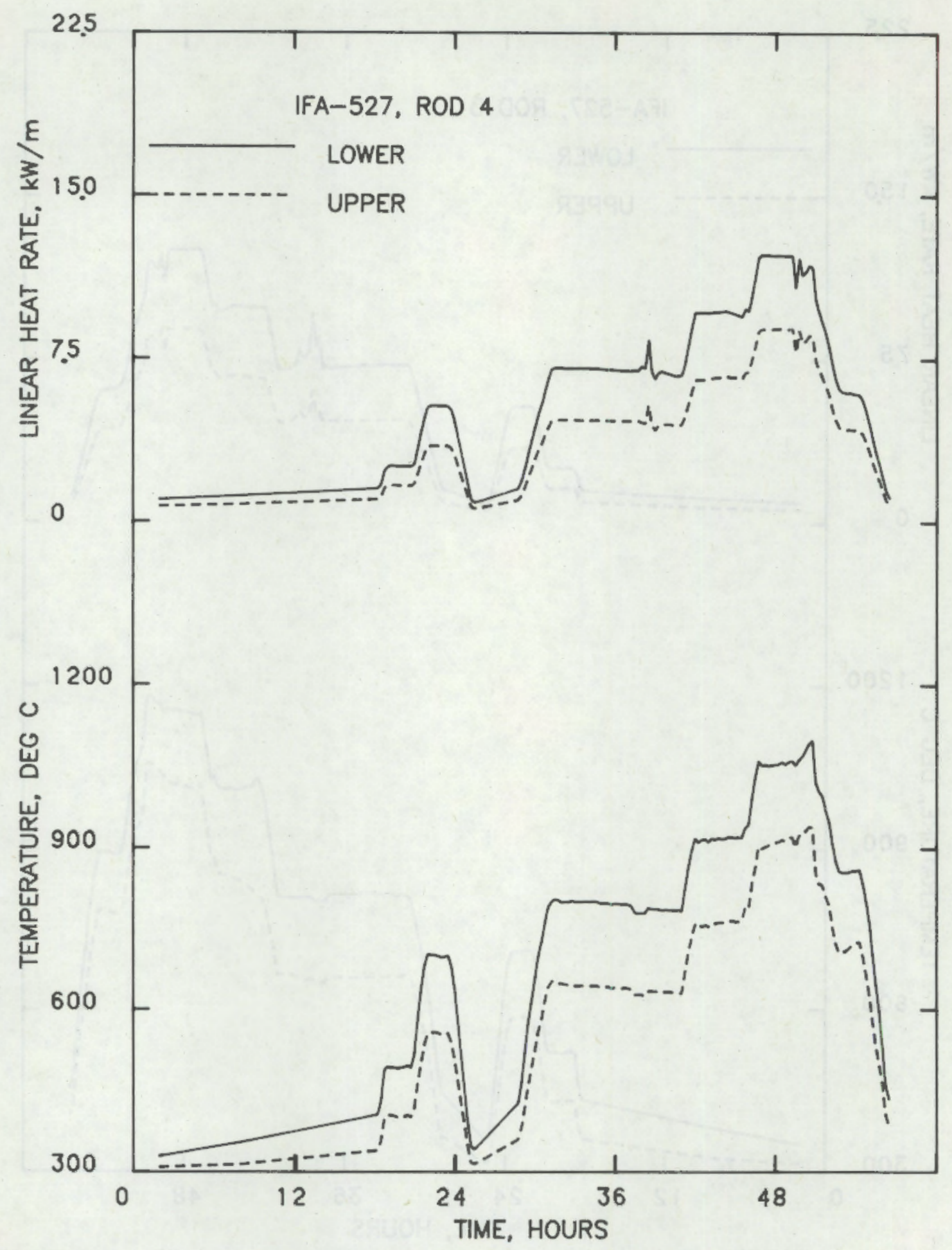

FIGURE 8. Upper and Lower Thermocouple Readings and Associated Linear Heat Ratings for Rod 4 of IFA-527; First Rise to Power 


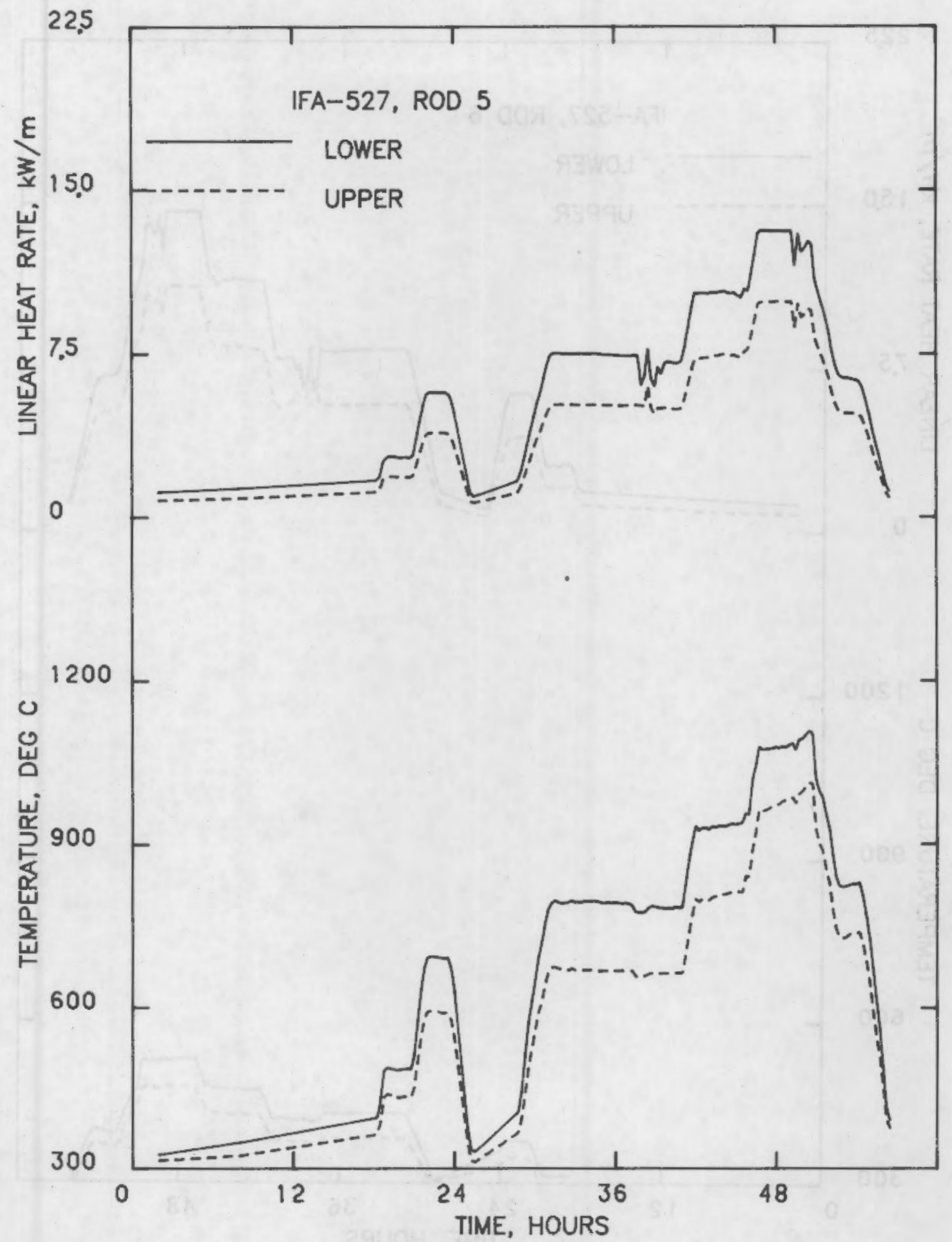

FIGURE 9. Upper and Lower Thermocouple Readings and Associated Linear Heat Ratings for Rod 5 of IFA-527; First Rise to Power 


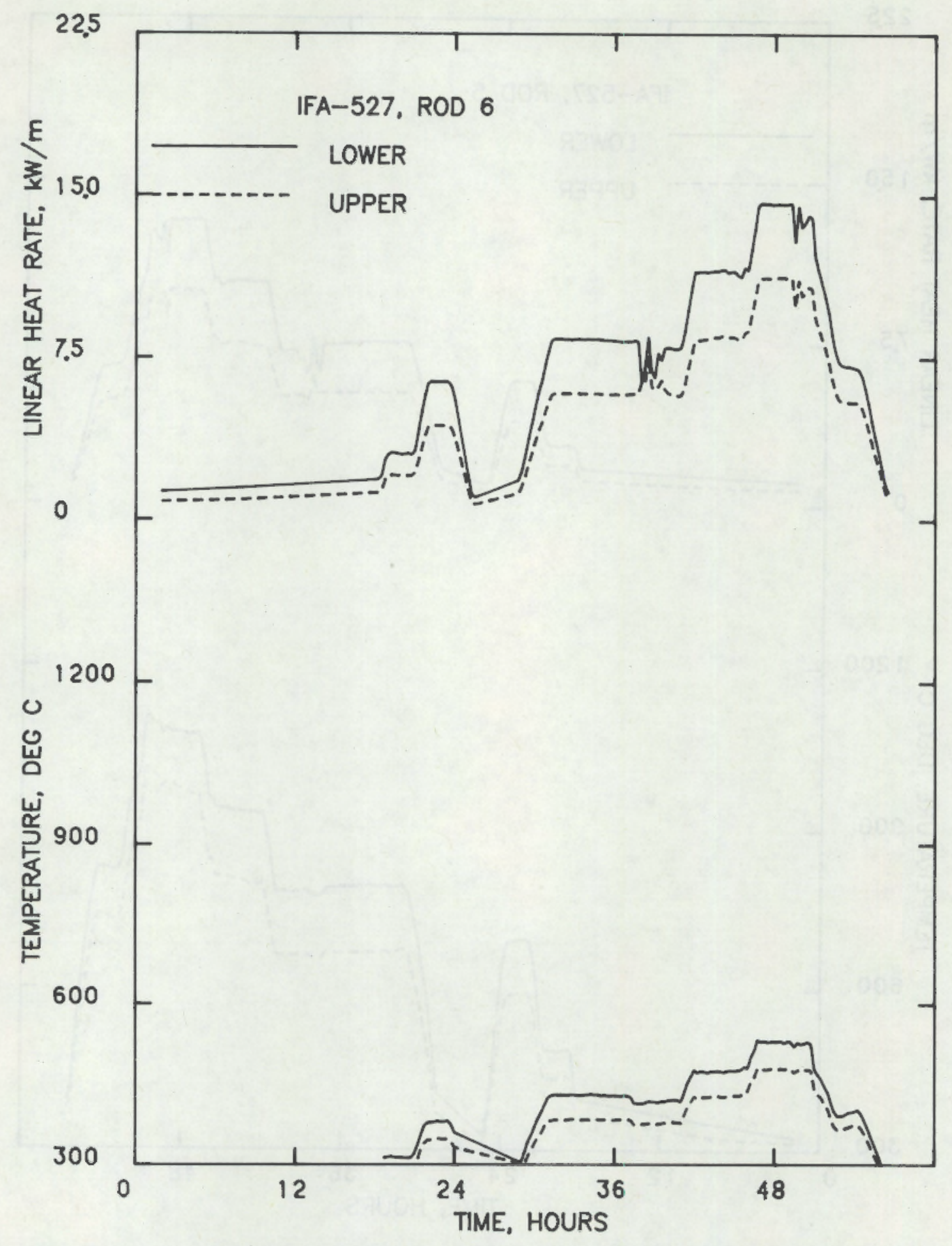

FIGURE 10. Upper and Lower Thermocouple Readings and Associated Linear Heat Ratings for Rod 6 of IFA-527; First Rise to Power 


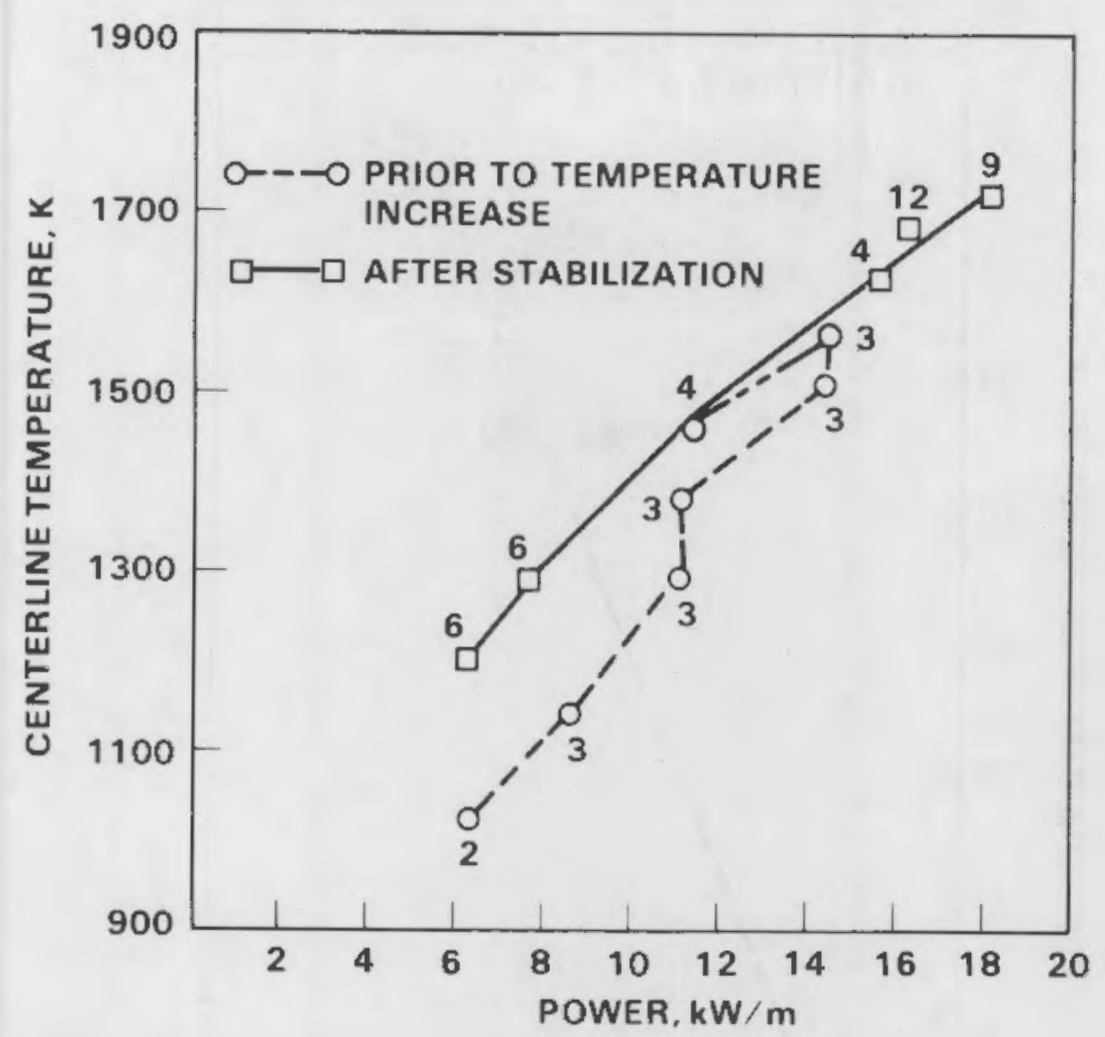

FIGURE 11. Temperature Versus Power for Lower Thermocouple of Rod 1 Through the First 12 Days of Irradiation (the numbers refer to corisecutive days since startup)

The magnitude of the resistance shifts is somewhat related to the temperatures attained by these rods. The temperature increment from the beginning to the end of the peak-power hold on July 3 versus the centerline temperature attained at the beginning of that hold is plotted in Figure 12. As can be seen, there is some pattern to the temperature increments among rods 1 through 5 , but there is a large difference between rods 1 through 5 and rod 6 (the small-gap rod). This "threshold" nature of the effect points to in-reactor densification as a probable cause of the temperature rise. Comparative Performance of Rods 1 Through 5

After temperature stabilization (that is, after July 5 ) the identically designed rods 1 through 5 (a11 with 230-um nominal as-fabricated diametral gaps) evidenced nearly identical temperature versus power curves. This is not necessarily a trivial result: The xenon fill gas in these rods should have 


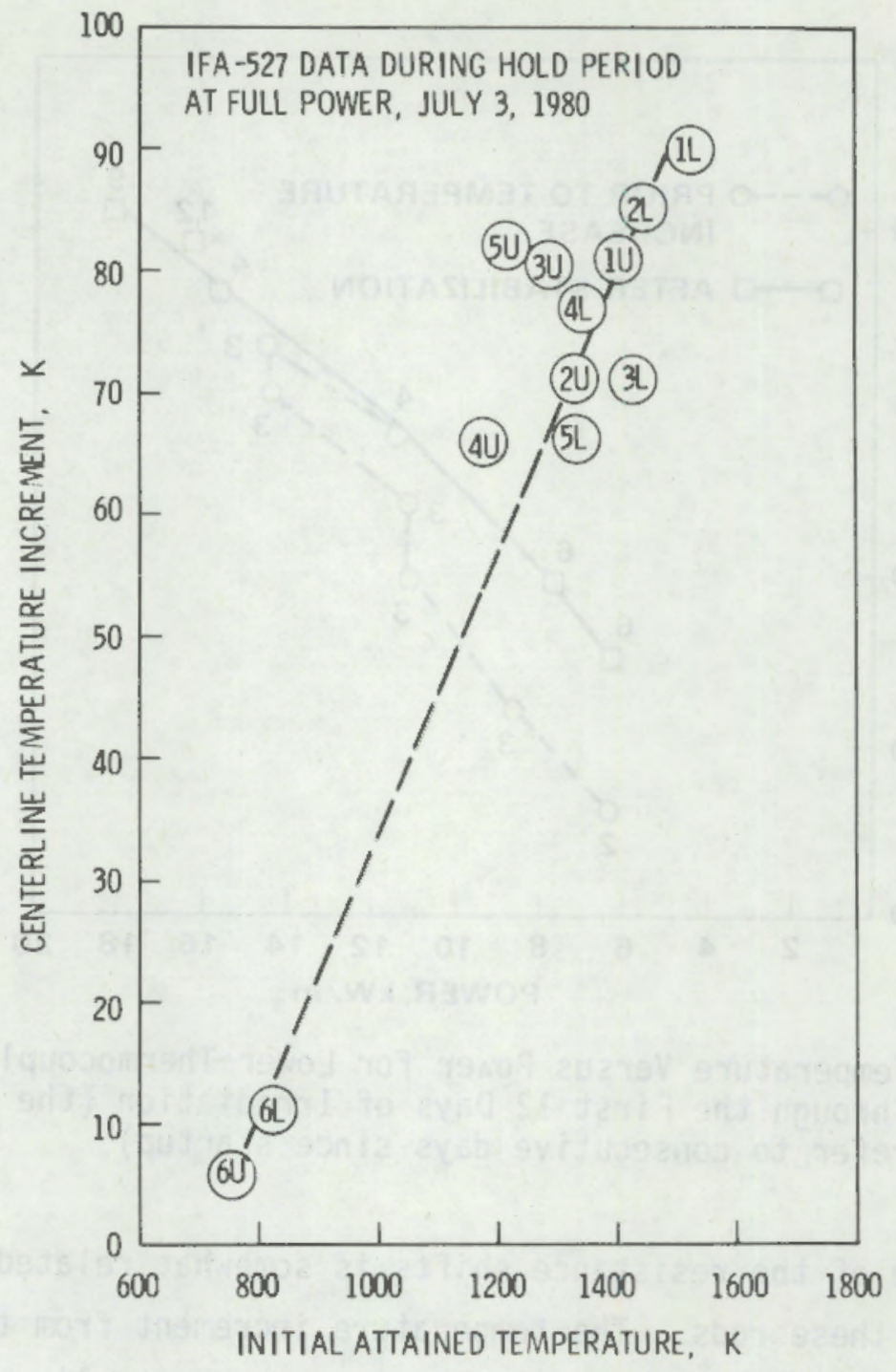

FIGURE 12. Temperature Increments Experienced During First Full-Power Hold Period Versus Initial Temperature at the Start of the Hold (1L, $1 \mathrm{U}$, etc., refer to rod number and thermocouple position)

greatly magnified significant variations in crack patterns or pellet fragment relocation. We can only conclude that there was little variation. Figure 13 shows temperature versus power behavior on July 9 for the lower thermocouples of rods 1 through 5 and the $(3 \sigma)$ uncertainty band for the calculated centerline temperature; a $10 \%$ relative uncertainty is assumed in both local linear heat generation rate and fuel thermal conductivity. Propagation of these uncertainties to the uncertainty in fuel centerline temperature was accomplished using the methods described by Cunningham et al. (1980). 


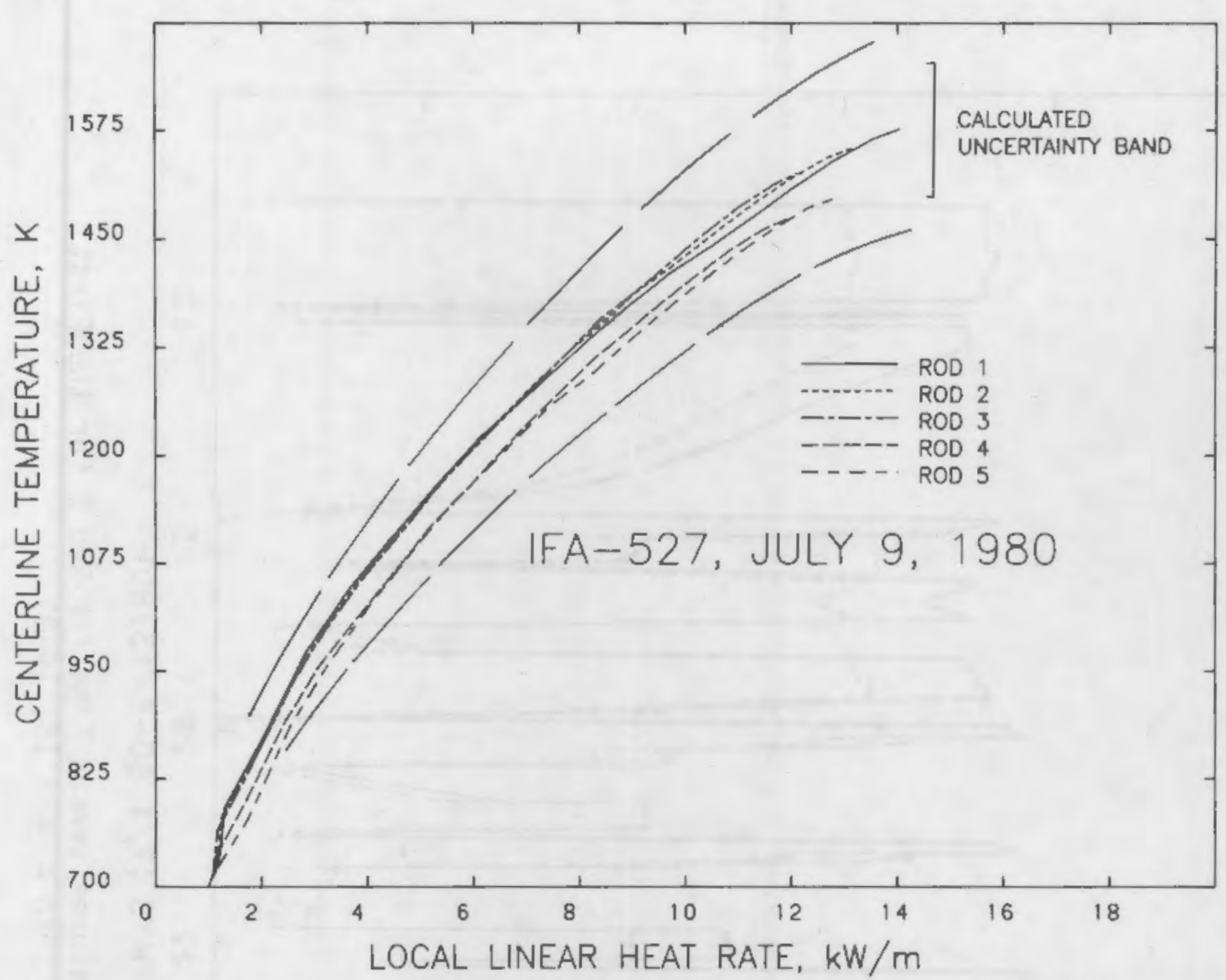

FIGURE 13. Stabilized Temperature Versus Power Data for Rods 1 Through 5 (230- $\mu \mathrm{m}$ gap rods) Relative to a Calculated Uncertainty Band

Temperature Behavior from July 5 to August 15,1980

Figures 14 through 19 show the temperature behavior history for IFA-527 rods throughout the initial irradiation period (July 1 to August 15, 1980). The total assembly power is shown in Figure 20 for reference. It is difficult to use these plots by themselves to find data trends; but a trend that does appear upon close inspection is a slight decrease in fuel temperature at a given power in the lower thermocouple site in the nominal-gap rods. This is emphasized in Figures 21 and 22; the excellent correspondence of the (stabilized) rod 1 temperature versus power curves on the July 8-9 approach to power and the end-to-end shifts in these curves evidenced by the approach to power on August 12 are shown. The overall decrease in fuel temperature is shown for the lower thermocouple of rod 1 in Figure 23, where the appropriate curves from 


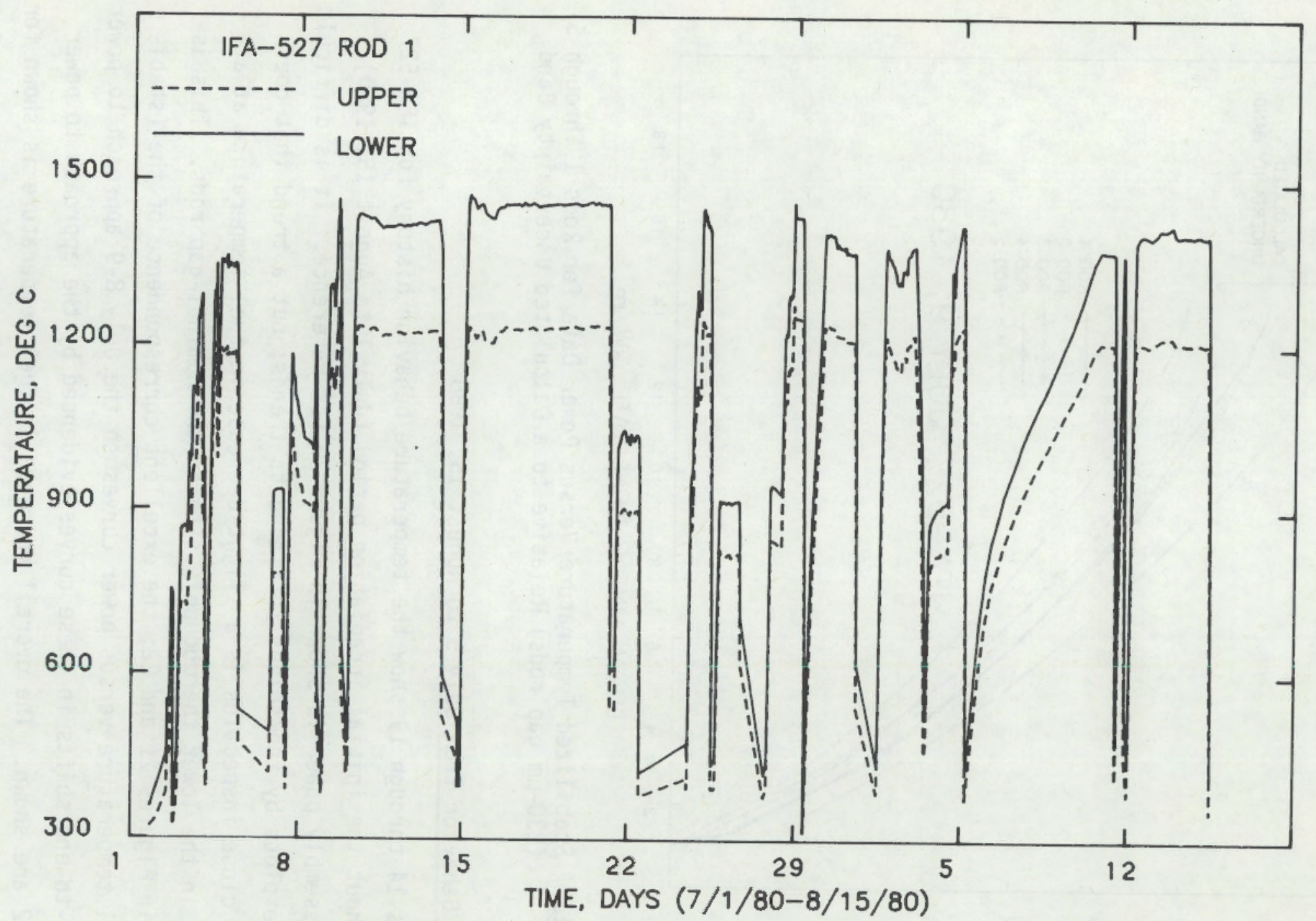

FIGURE 14. Fuel Centerline Temperatures Versus Time for Rod 1 for the First Operating Period (July 1 to August 15, 1980) 


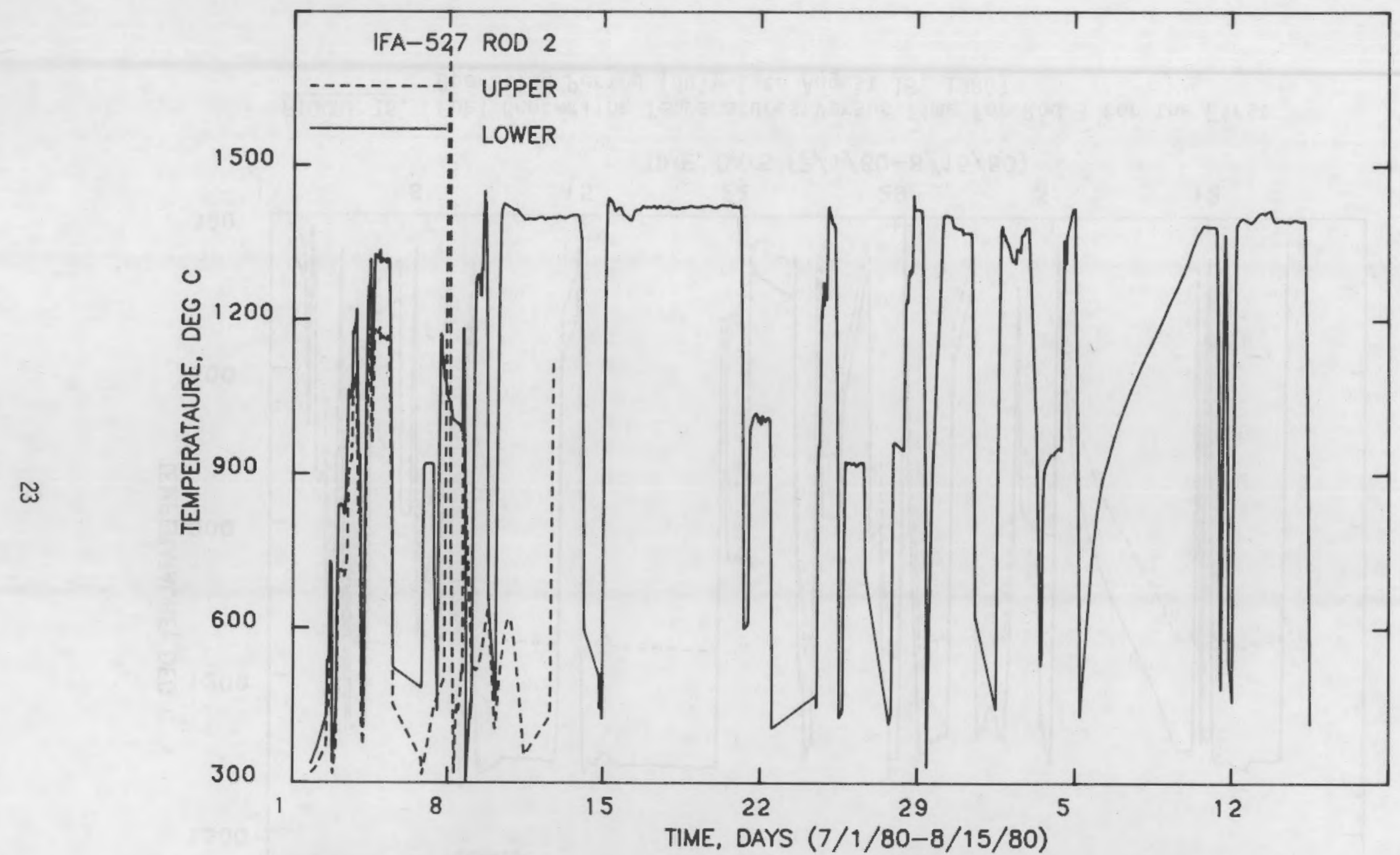

FIGURE 15. Fuel Centerline Temperatures Versus Time for Rod 2 for the First Operating Period (July 1 to August 15, 1980) 


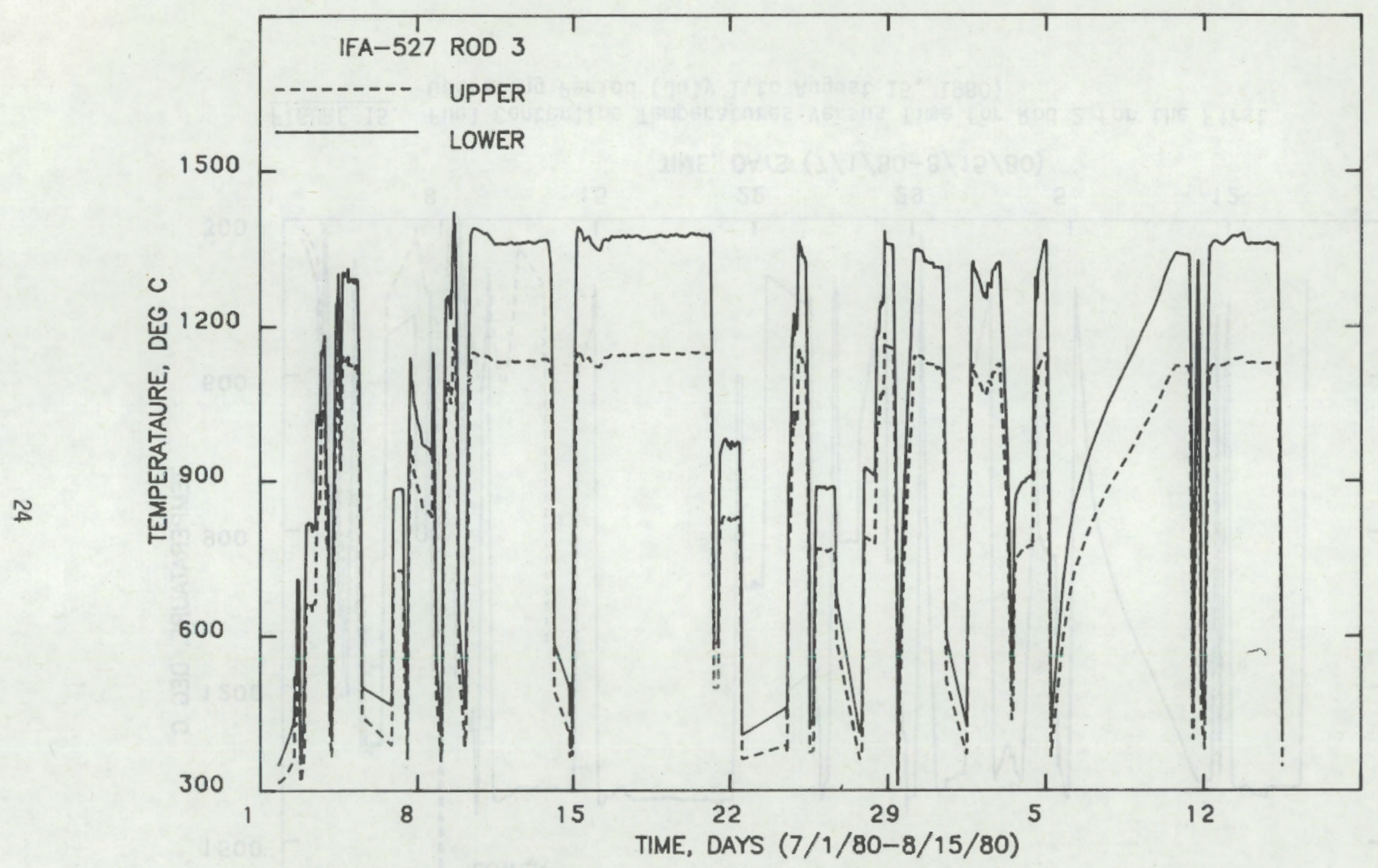

FIGURE 16. Fuel Centerline Temperatures Versus Time for Rod 3 for the First Operating Period (July 1 to August 15, 1980) 


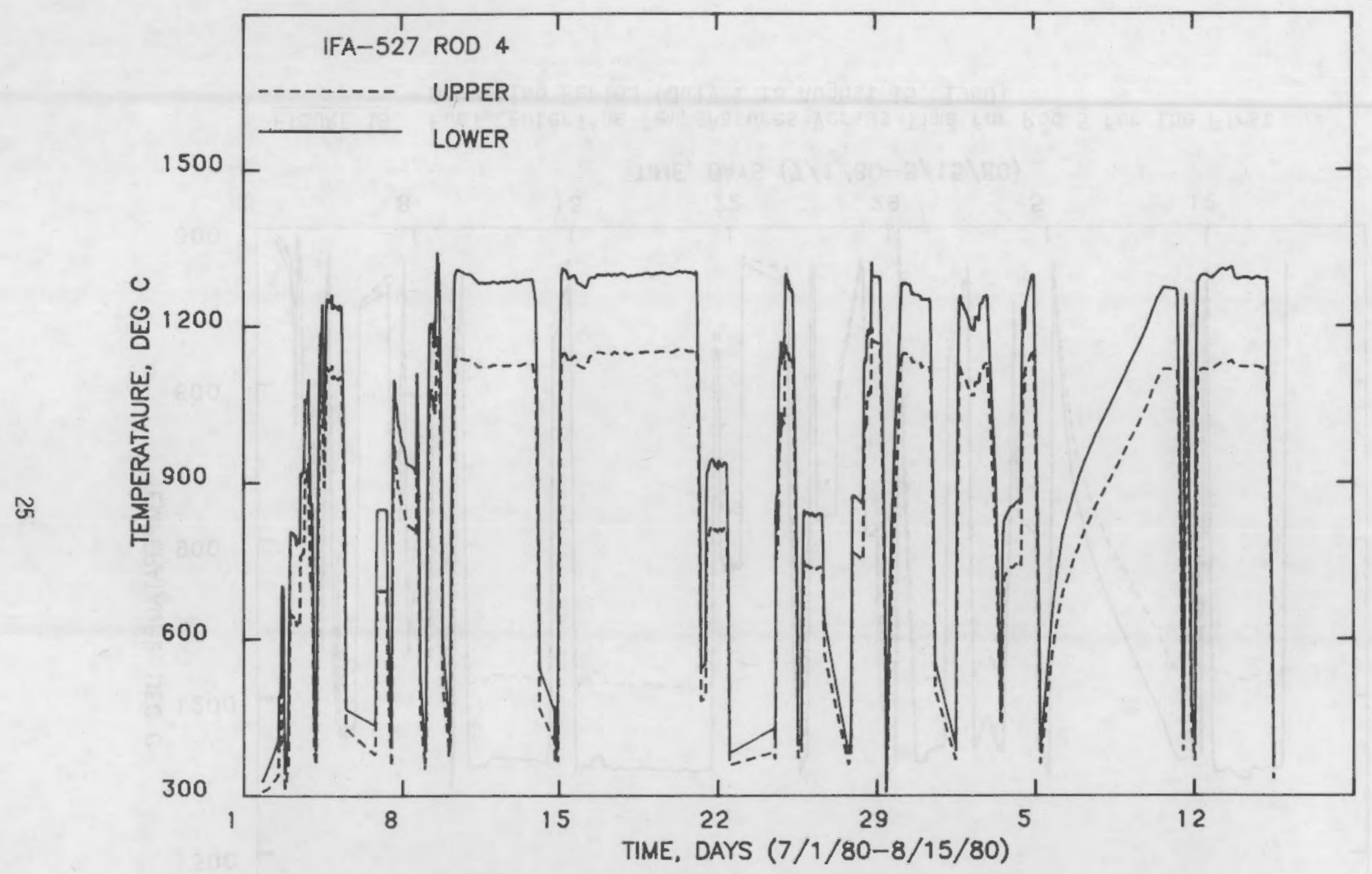

FIGURE 17. Fuel Centerline Temperatures Versus Time for Rod 4 for the First Operating Period (July 1 to August 15, 1980) 


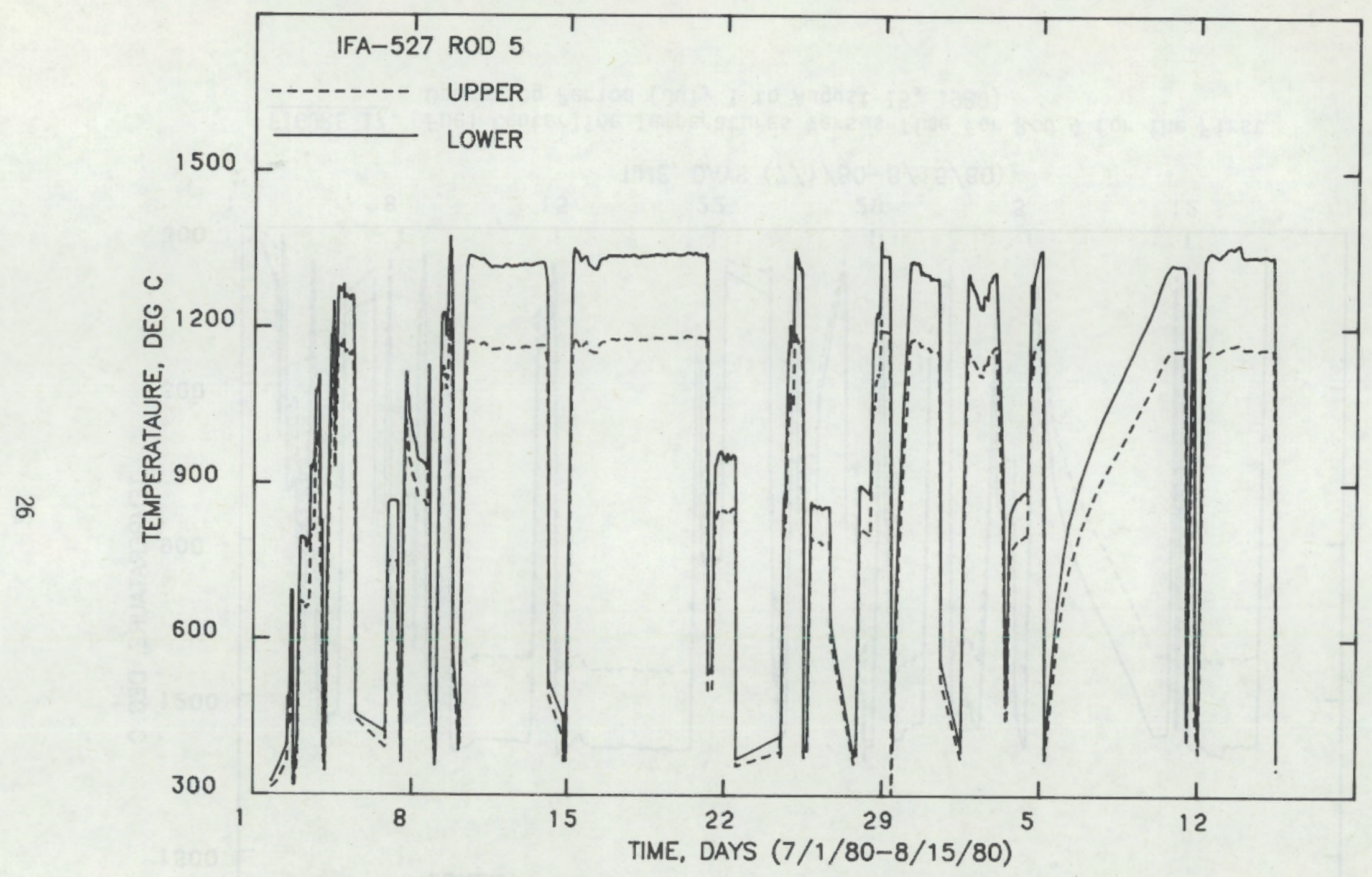

FIGURE 18. Fuel Centerline Temperatures Versus Time for Rod 5 for the First Operating Period (July 1 to August 15, 1980) 


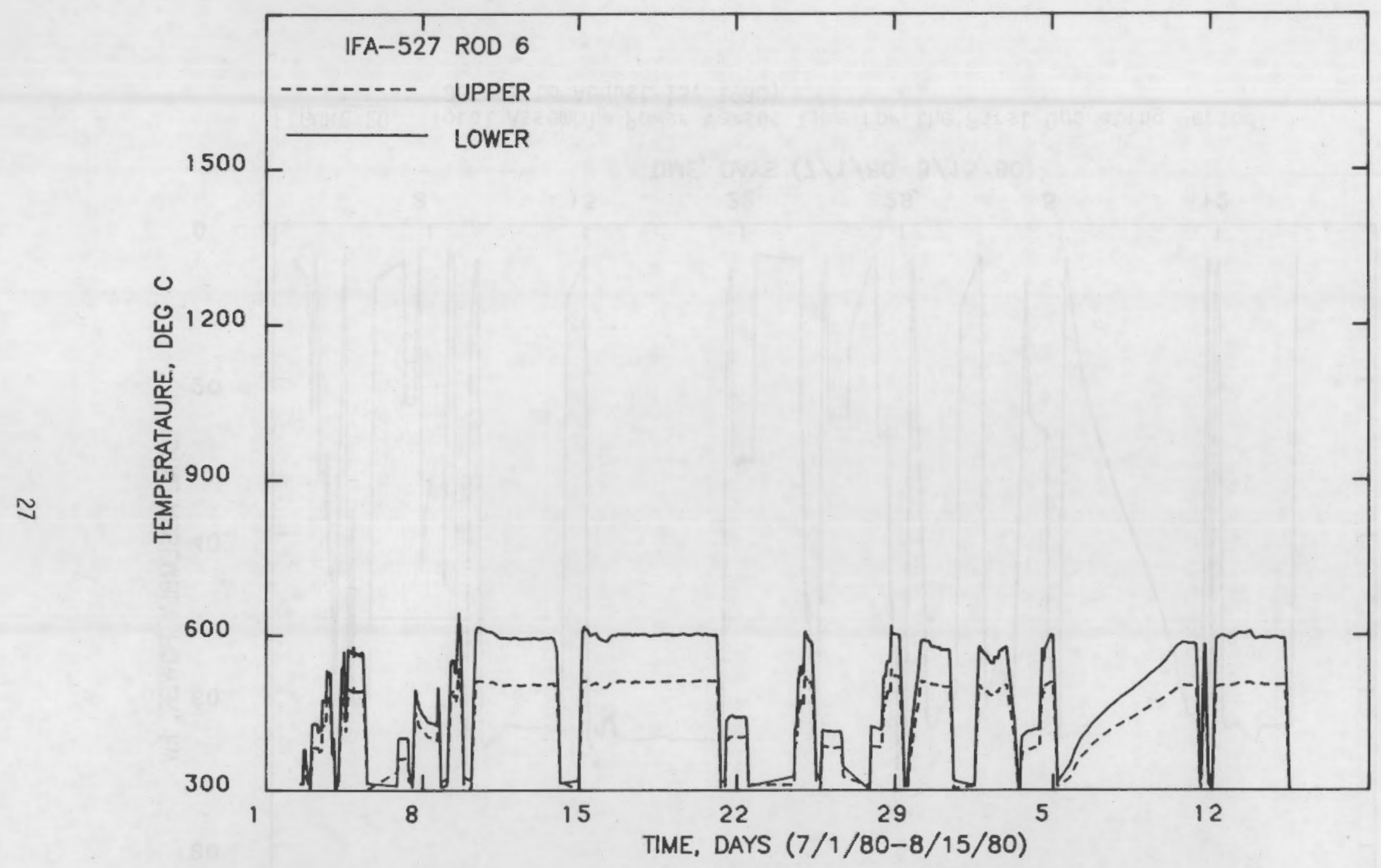

FIGURE 19. Fuel Centerline Temperatures Versus Time for Rod 6 for the First Operating Period (July 1 to August 15, 1980) 


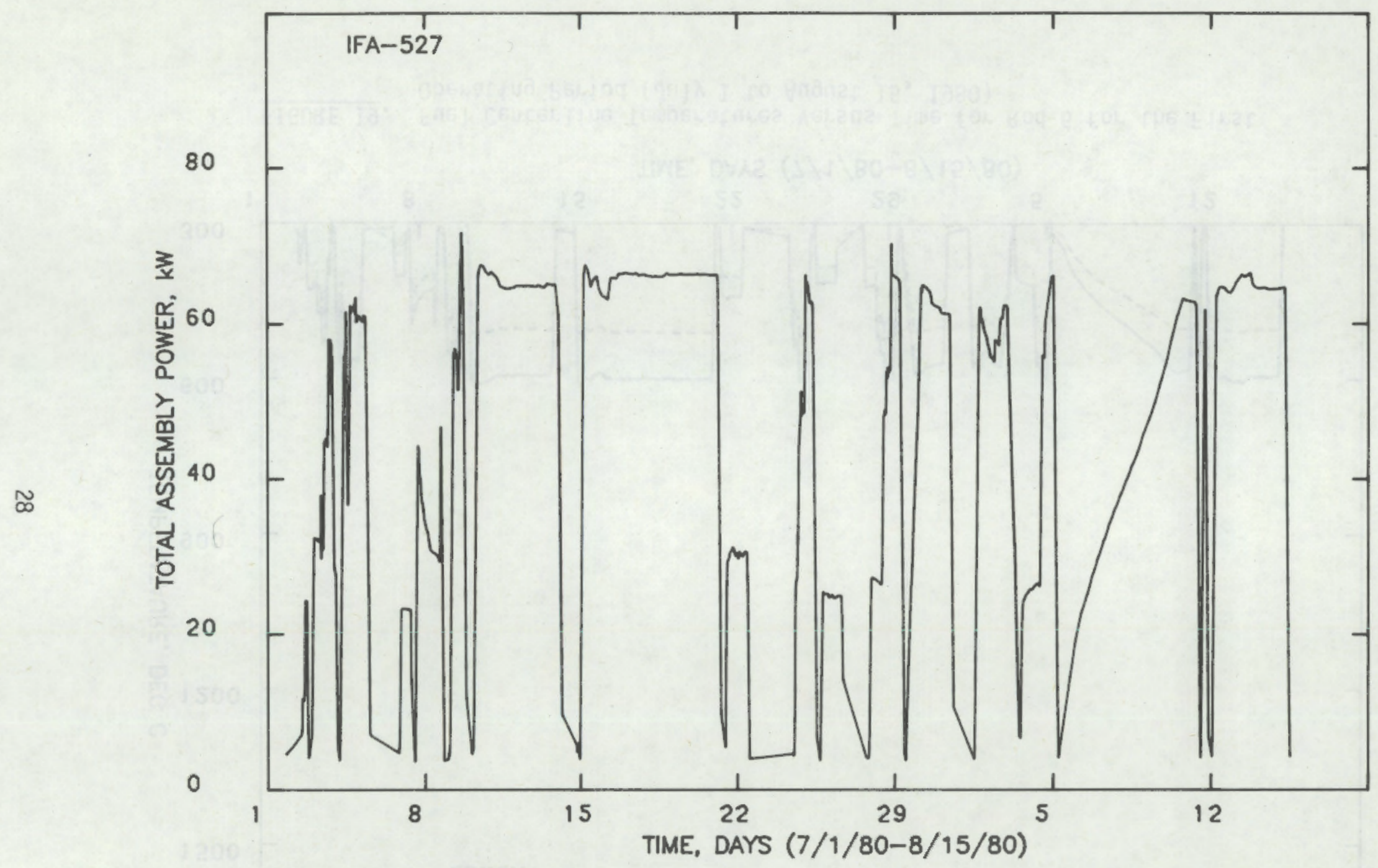

FIGURE 20. Total Assembly Power Versus Time for the First Operating Period (July 1 to August 15, 1980) 


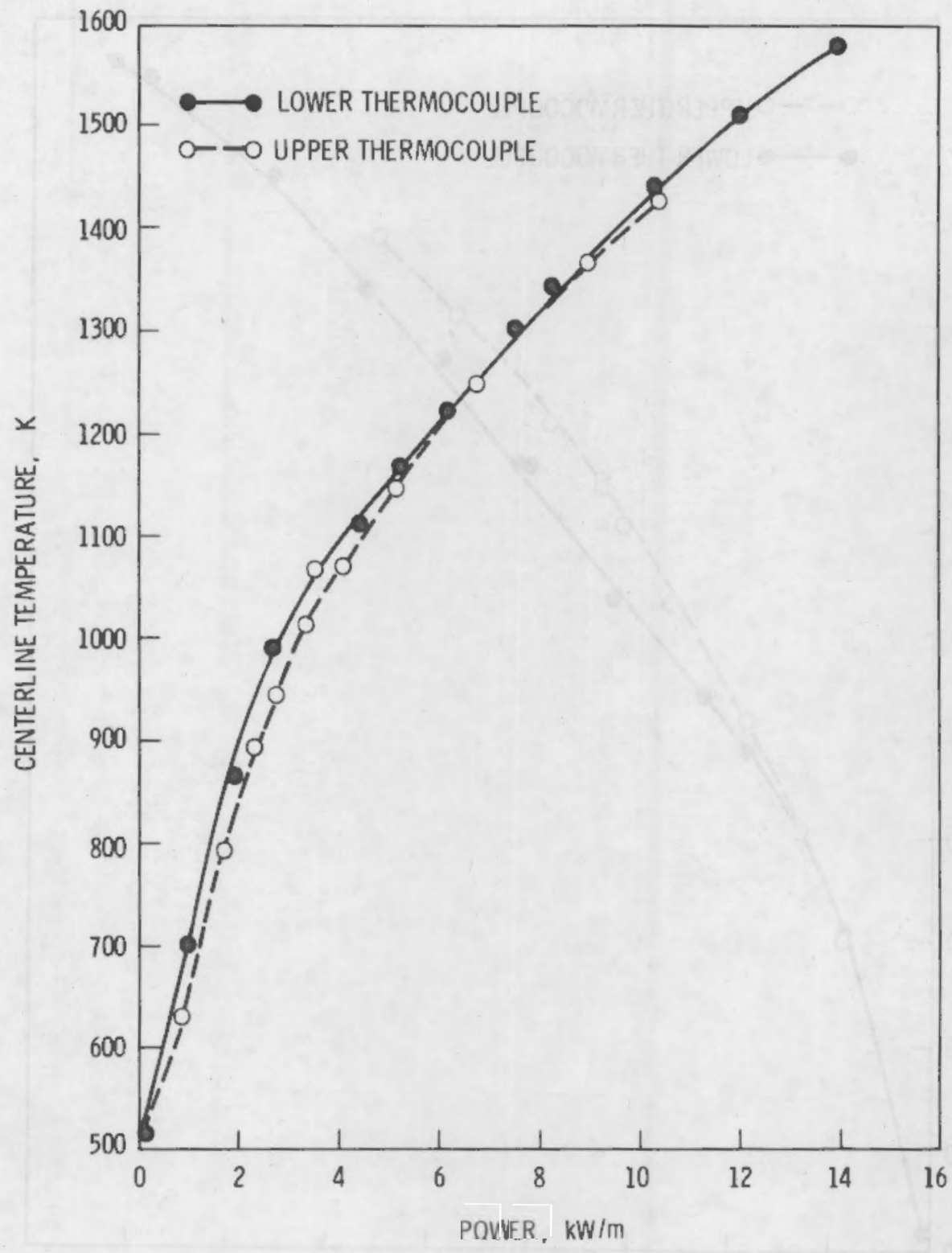

FIGURE 21. Stabilized Upper/Lower Fuel Centerline Temperatures Versus Local Linear Heat Rating for Rod 1 on July 8 and 9, 1980 


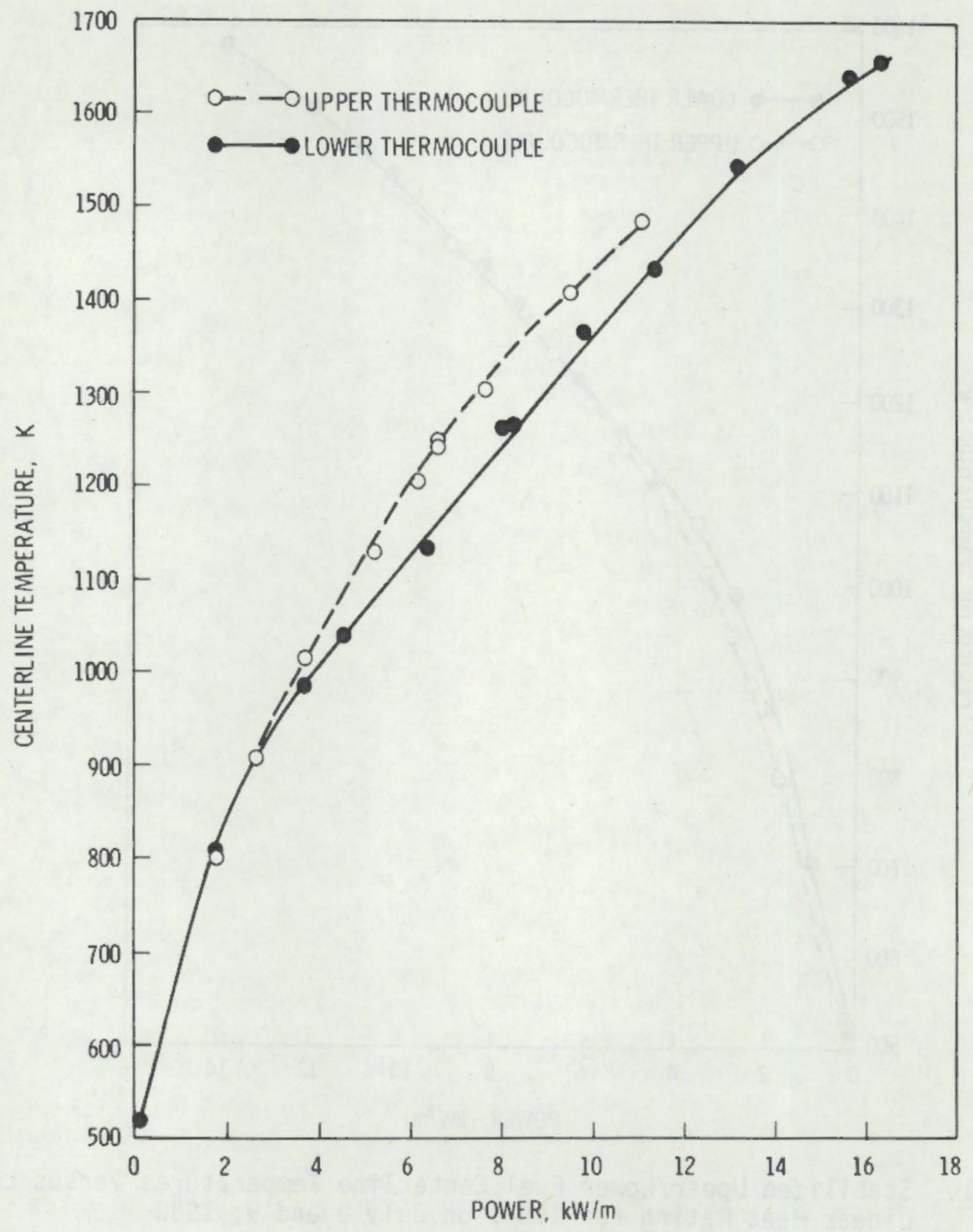

FIGURE 22. Stabilized Upper/Lower Fuel Centerline Temperatures Versus Local Linear Heat Rating for Rod 1 on August 12, 1980 


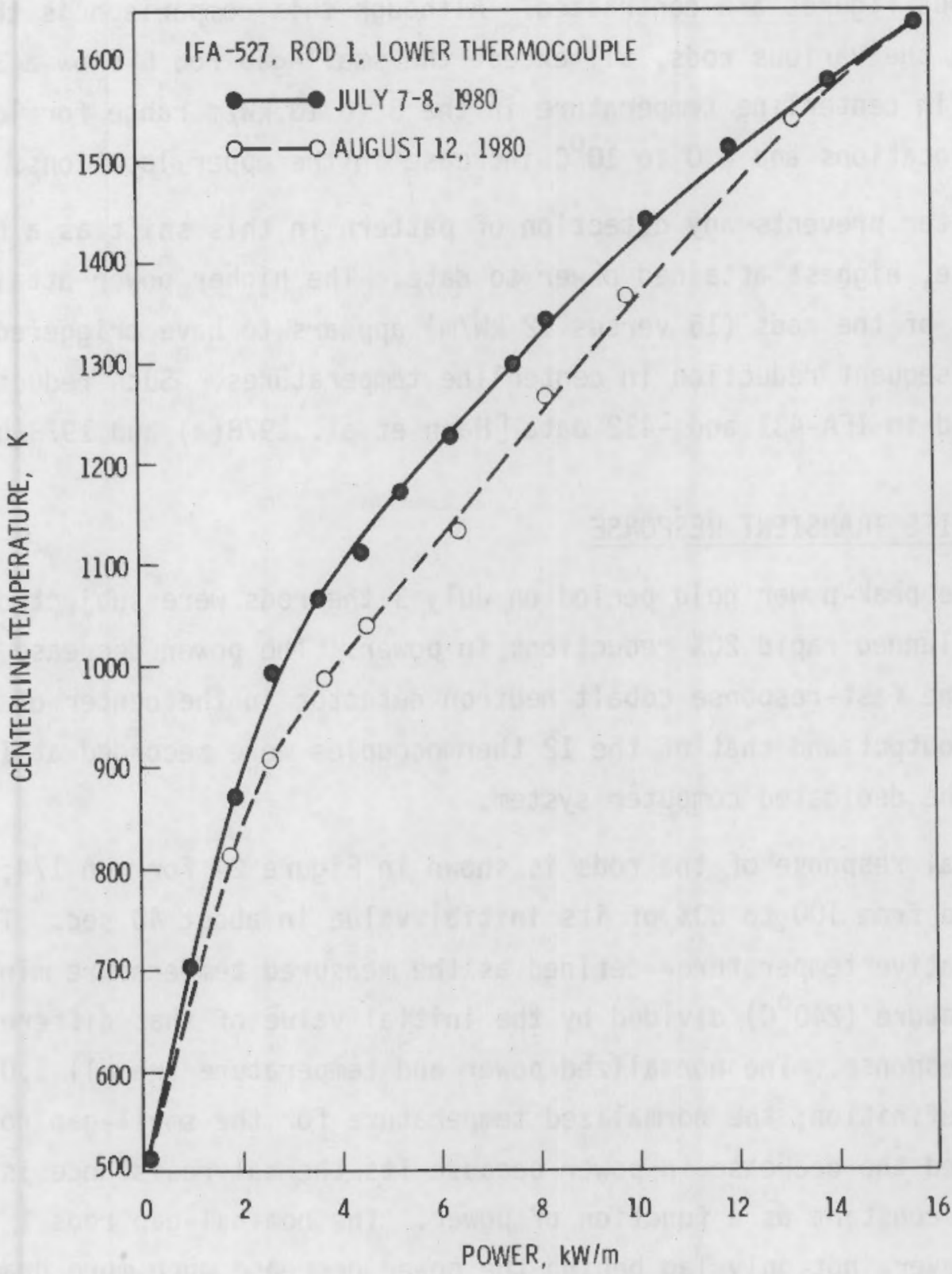

FIGURE 23. Lower Thermocouple Readings for Rod 1 Versus Linear Heat Rating on July 8 and August 12, 1980 
the two previous figures are contrasted. Although this comparison is the most dramatic among the various rods, all except the small-gap rod 6 show a 30 to $60^{\circ} \mathrm{C}$ decrease in centerline temperature in the 8 to $10 \mathrm{~kW} / \mathrm{m}$ range for lower thermocouple locations and a 0 to $10^{\circ} \mathrm{C}$ increase in the upper locations.

Data scatter prevents any detection of pattern in this shift as a function of, for example, highest attained power to date. The higher power attained by the lower ends of the rods (15 versus $12 \mathrm{~kW} / \mathrm{m}$ ) appears to have triggered relocation and consequent reduction in centerline temperatures. Such reductions were also noted in IFA-431 and -432 data [Hann et al. 1978(a) and 1978(b)].

\section{BEGINNING-OF-LIFE TRANSIENT RESPONSE}

During the peak-power hold period on July 3 the rods were subjected to a series of preplanned rapid 20\% reductions in power. The power decrease was monitored by the fast-response cobalt neutron detector in the center of the assembly; its output and that of the 12 thermocouples were recorded at $1-\mathrm{Hz}$ frequency by the dedicated computer system.

The typical response of the rods is shown in Figure 24 for run 174; the power decreased from 100 to $80 \%$ of its initial value in about $40 \mathrm{sec}$. The normalized relative temperature--defined as the measured temperature minus the coolant temperature $\left(240^{\circ} \mathrm{C}\right)$ divided by the initial value of that difference-decreased in response. The normalized power and temperature are all 1.00 at time zero by definition; the normalized temperature for the small-gap rod 6 closely followed the decrease in power because its thermal resistance is small and relatively constant as a function of power. The nominal-gap rods 1 through 5, however, not only lag behind the power decrease much more dramatically but also fail to approximate its slope due to the fact that the thermal resistance of these rods is high and sharply dependent on the power/temperature level. This qualitative correspondence of transient and steady-state behavior has been thoroughly discussed in previous reports. (a)

(a) See Lanning et al. 1979 and Lanning 1978. 


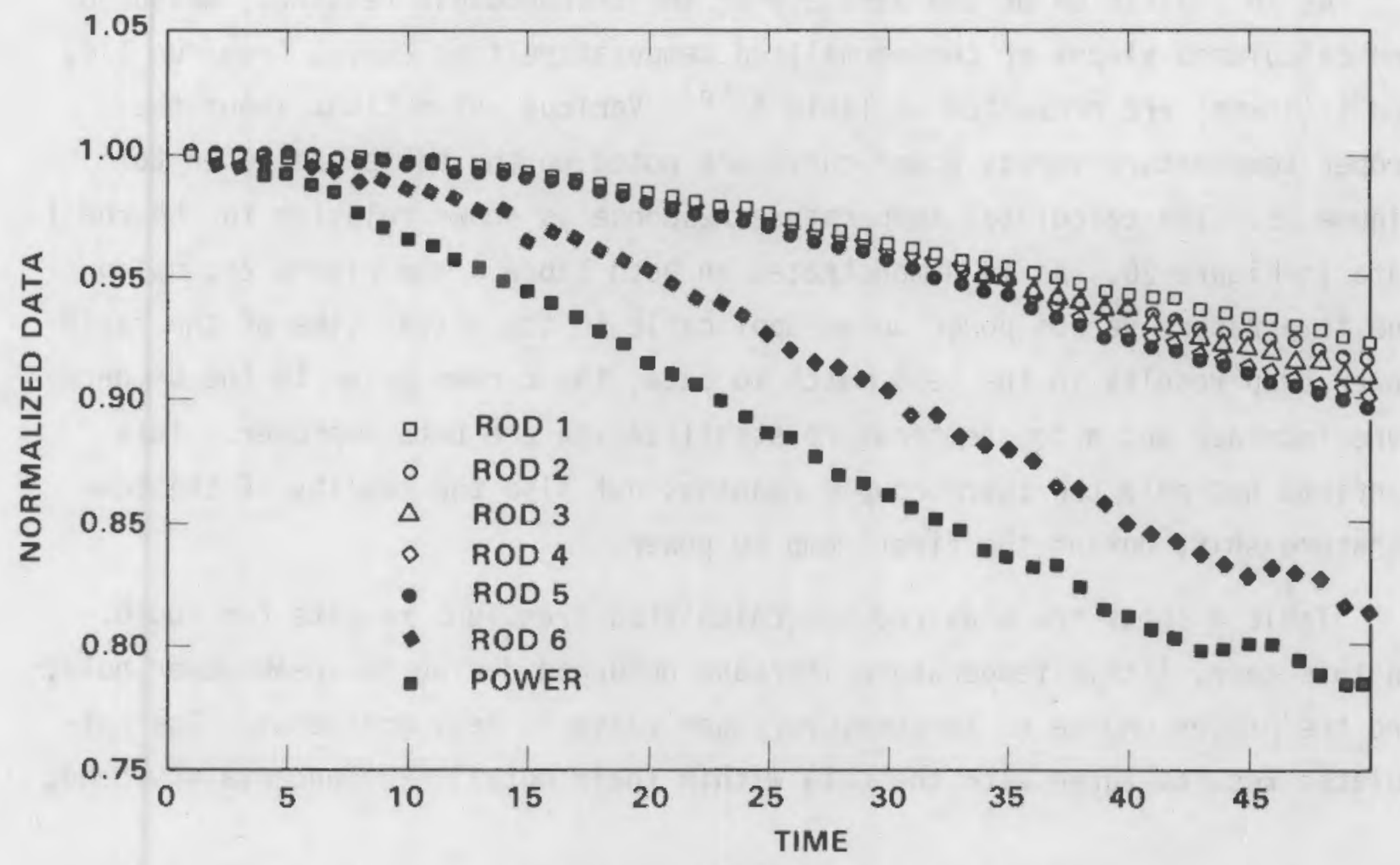

FIGURE 24. Normalized Power and Fuel Centerline Thermocouple Data for Linear Power Decrease (Run 174, July 3, 1980)

The divergence among rods 1 through 5 past about the 25-sec mark in Figure 24 is interesting; it corresponds well with the temperature versus power plot in Figure 13. The temperature decreases slowest in the higher resistance rods $(1,2$, and 3$)$ and fastest in the lower resistance rods (4 and 5).

A method has been previously proposed to confirm thermocouple accuracy using the transient response of the fuel temperatures. The ratio of the normalized temperature and power slopes in computerized simulations of these linear power decreases seems particularly insensitive to the modeling details used to make the calculation, provided the models are all "tuned" to match the same steady-state performance. So the argument goes that significant divergence between thermocouple performance and tuned calculations indicates a bias in the steady-state data and hence in the themocouple readings (Lanning 1979). 
As an indication of the adequacy of the thermocouple readings, measured and calculated slopes of the normalized temperature/time curves from run 174, rod 1 (lower) are presented in Table 3. (a) Various assumptions about the proper temperature versus power curve are noted on the table and refer to Figure 25. The calculated temperature response is shown relative to the rod 1 data in Figure 26. As is demonstrated in both Table 3 and Figure 26, using the temperature versus power curve applicable to the actual time of the rapid power drop results in the best match to data; the curves prior to the temperature increase and after temperature stabilization are both improper. This confirms not only the thermocouple readings but also the reality of the temperature shift during the first ramp to power.

Table 4 shows the measured and calculated transient results for rod 6 . In this case, little temperature increase occurred during the peak-power hold, and the proper choice of temperature/power curve is less ambiguous. The calculated results agree with the data within their mutual $(2 \sigma)$ uncertainty band.

TABLE 3. Measured and Calculated Temperature Slopes for Rod 1 (Lower), Run 174

\begin{tabular}{clc} 
Case & \multicolumn{1}{c}{ Assumptions } & $\begin{array}{c}\text { Slope of Temperature } \\
\text { Versus Time Curve, } \\
\% / \mathrm{sec}( \pm 2 \sigma)\end{array}$ \\
\cline { 2 - 3 } 1 & $\begin{array}{l}\text { Steady-state performance prior to } \\
\text { temperature increase }\end{array}$ & $0.20 \pm 0.01$ \\
2 & $\begin{array}{l}\text { Steady-state performance after the } \\
\text { first ramp }\end{array}$ & $0.27 \pm 0.01$ \\
3 & $\begin{array}{l}\text { Steady-state performance on first } \\
\text { ramp after temperature increase }\end{array}$ & $0.20 \pm 0.01$
\end{tabular}

(a) These calculations were performed using a constant gap conductance assumption in the MWRAM code (Lanning 1978). 


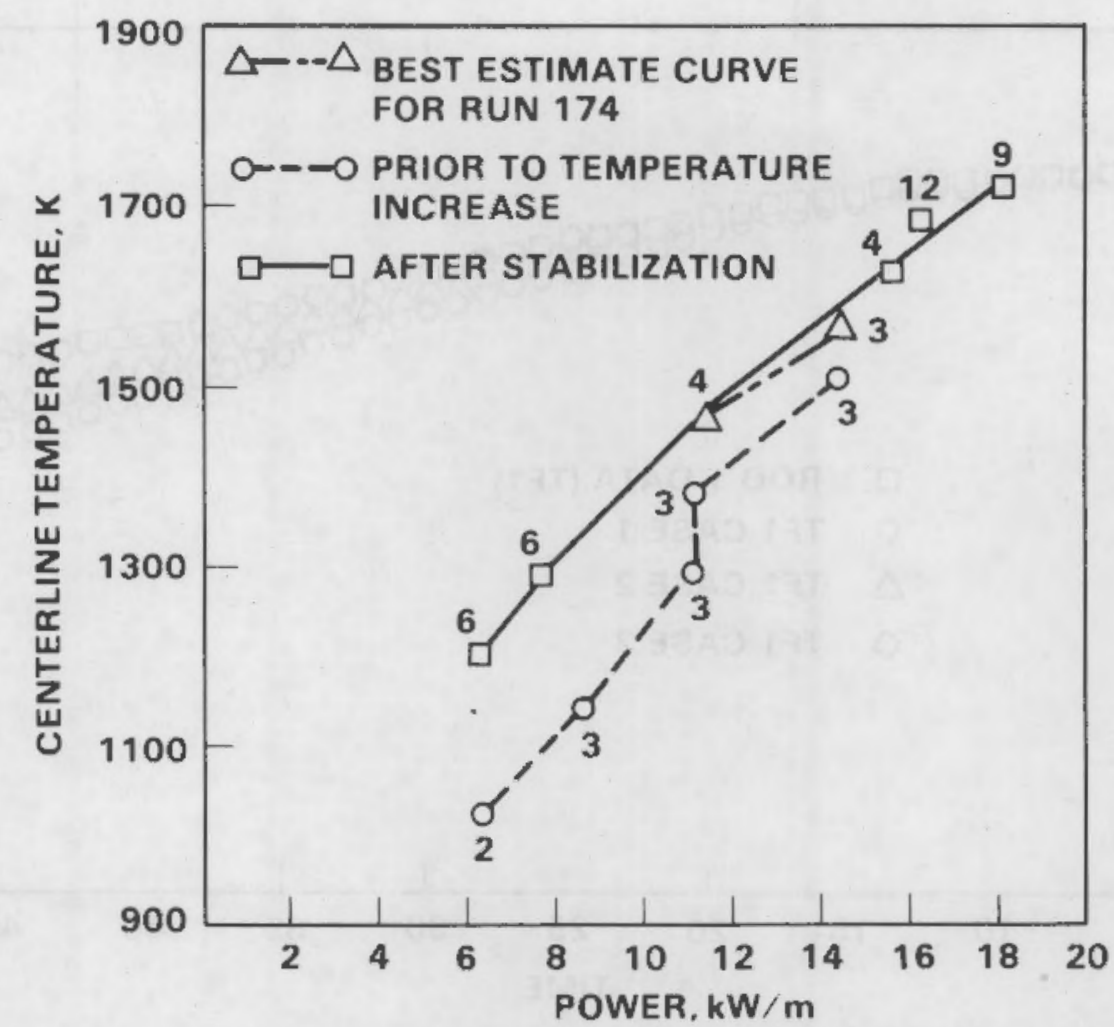

FIGURE 25. Temperature Versus Power for Rod I (Lower) from July 1-12, 1980, Showing MWRAM Best-Estimate Input Conditions for Run 174 Simulation (numbers refer to consecutive days of irradiation) 


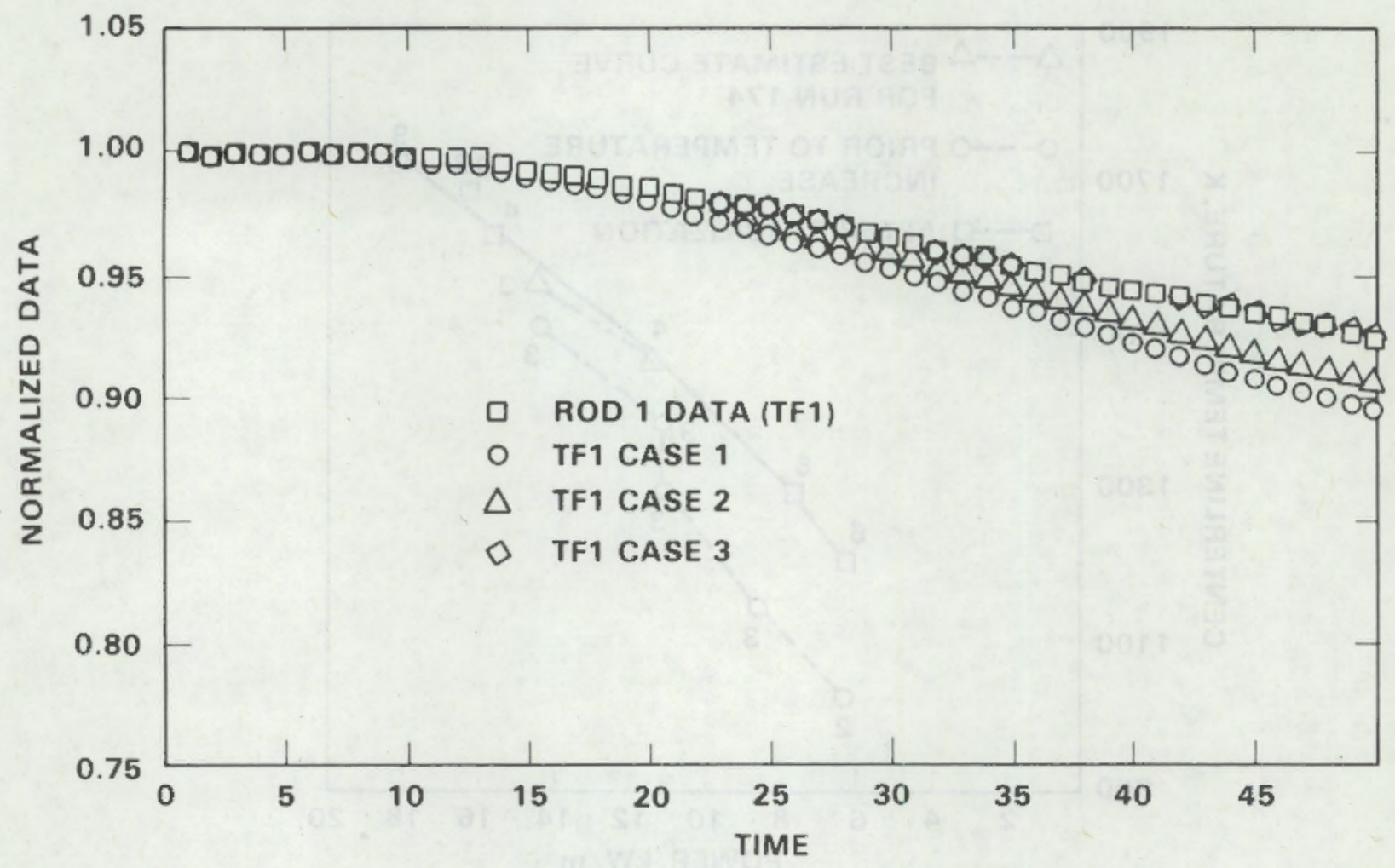

FIGURE 26. Measured and MWRAM Calculated Fuel Centerline Temperature Response for Run 174 for Rod 1 (Lower Thermocouple)

TABLE 4. Measured and Calculated Temperature Slopes for Rod 6, Run 174

\begin{tabular}{cccc} 
Thermocouple & $\begin{array}{c}\text { Measured Slope of Normalized } \\
\text { Temperature Versus Time, } \\
\% / \mathrm{sec}( \pm 2 \sigma)\end{array}$ & $\begin{array}{c}\text { Calculated } \\
\text { Temperature Slope, } \\
\% / \mathrm{sec}( \pm 2 \sigma)\end{array}$ \\
\cline { 2 - 3 } $\begin{array}{c}0.49 \pm 0.01 \\
\text { Upper }\end{array}$ & $0.49 \pm 0.02$ & & $0.44 \pm 0.03$ \\
Lower & $0.46 \pm 0.03$
\end{tabular}

SUMMARY OF PREFAILURE THERMAL PERFORMANCE

Throughout the first month of operation, the thermal behavior of the IFA-527 rods was very similar to previously analyzed Halden test rods: A significant temperature increase at constant power during first startup was followed in the higher powered lower sections by a gradual decline in temperature at a given power. All rods of identical design behaved nearly the same, and the steady-state behavior seems qualitatively well confirmed by the transient behavior observed in $20 \%$ power decreases. 


\section{PREFAILURE CLADDING ELONGATION AND GAS PRESSURE DATA}

The general trends noted in the previous section for themal behavior of the rods in the first month of operation are well correlated to their elongation and pressure data: The rods of identical design (rods 1 through 5) are tightly grouped, and the small-gap rod 6 is remarkably different.

\section{CLADDING ELONGATION DATA}

Rod axial elongation will be examined in this section from the standpoints of first ramp data, behavior during several subsequent ramps, and overall behavior during the first one and one-half months of operation.

\section{Initial Ramp Data}

Figures 27 through 32 show the elongation behavior versus time for the six rods during the first rise to power and the rod-averaged linear heat ratings. Two features can be seen in these curves: a strange increase in elongation (during the hold period at 7.0 to $7.5 \mathrm{~kW} / \mathrm{m}$ ) while the power was holding constant and the offset of approximately $60 \mu \mathrm{m}$ at the shutdown after the first rise to full power.

The first feature is apparently related to subcooling water flow and consequent coolant temperature changes, which affect the assembly stay rod expansion and hence the apparent rod elongation. The measured rod elongation is actually the difference between the stay rod length (presumed constant) and the rod length since the elongation detectors ride on the bottom assembly grid and the rods hang "free" from the top assembly grid; the two grids are connected by the stay rods. The elongation detector readings are calibrated (brought to zero by added constants) when the coolant water is at $240^{\circ} \mathrm{C}$. However, subcooled water admitted for assembly power calibration is 10 to $15^{\circ} \mathrm{C}$ cooler, which causes a shortening of the stay rods and a false apparent increase in the rod length. This effect can be seen very clearly by comparing Figures 27 through 32 with Figure 33 , which shows the increase in temperature across the assembly channel plotted versus time over the same time range covered in Figures 27 through 32 . Normally, this temperature rise is 1 to $3^{\circ} \mathrm{C}$; when the 


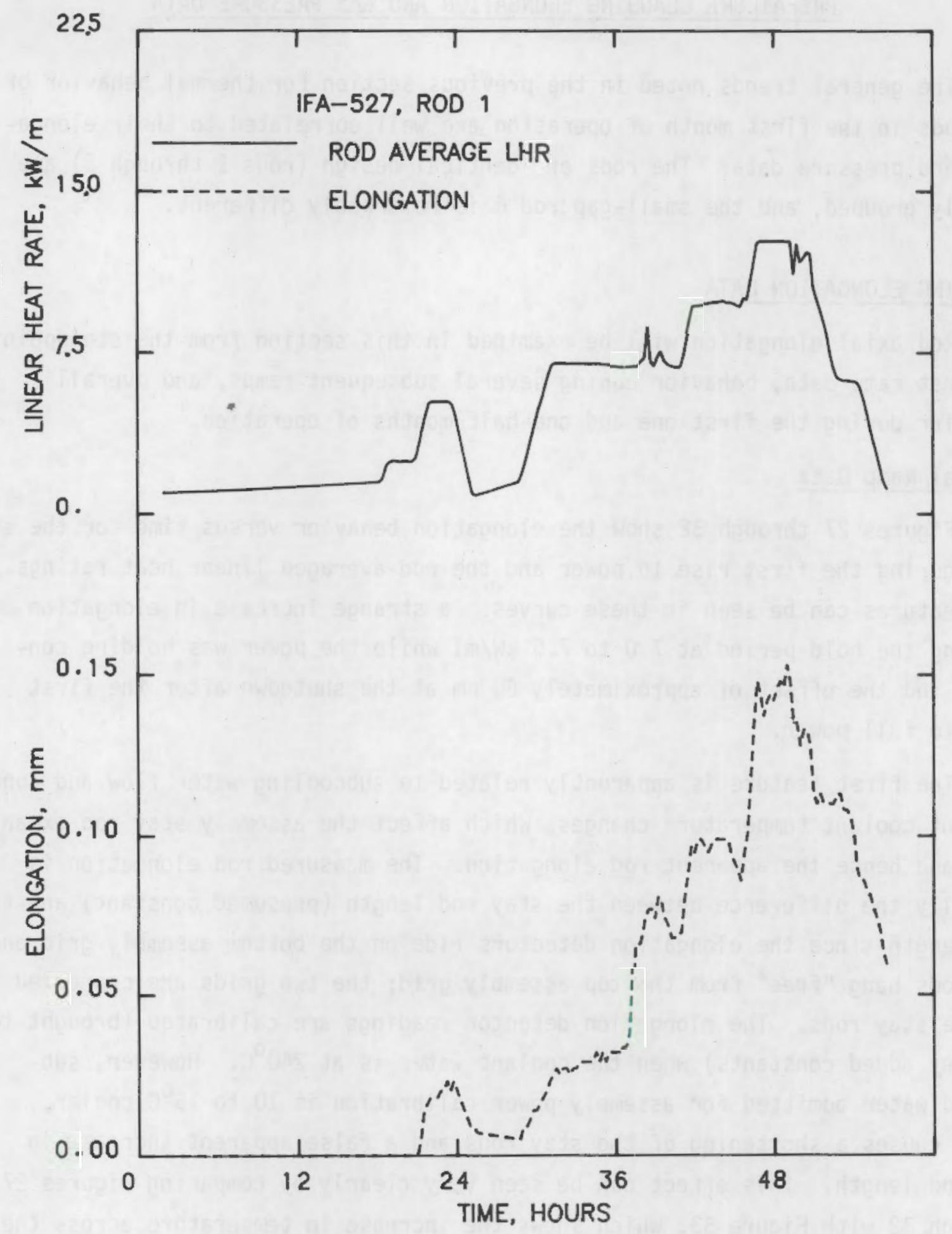

FIGURE 27. Fuel Rod Elongation and Average Linear Heat Rating for Rod 1 During the First Rise to Power 


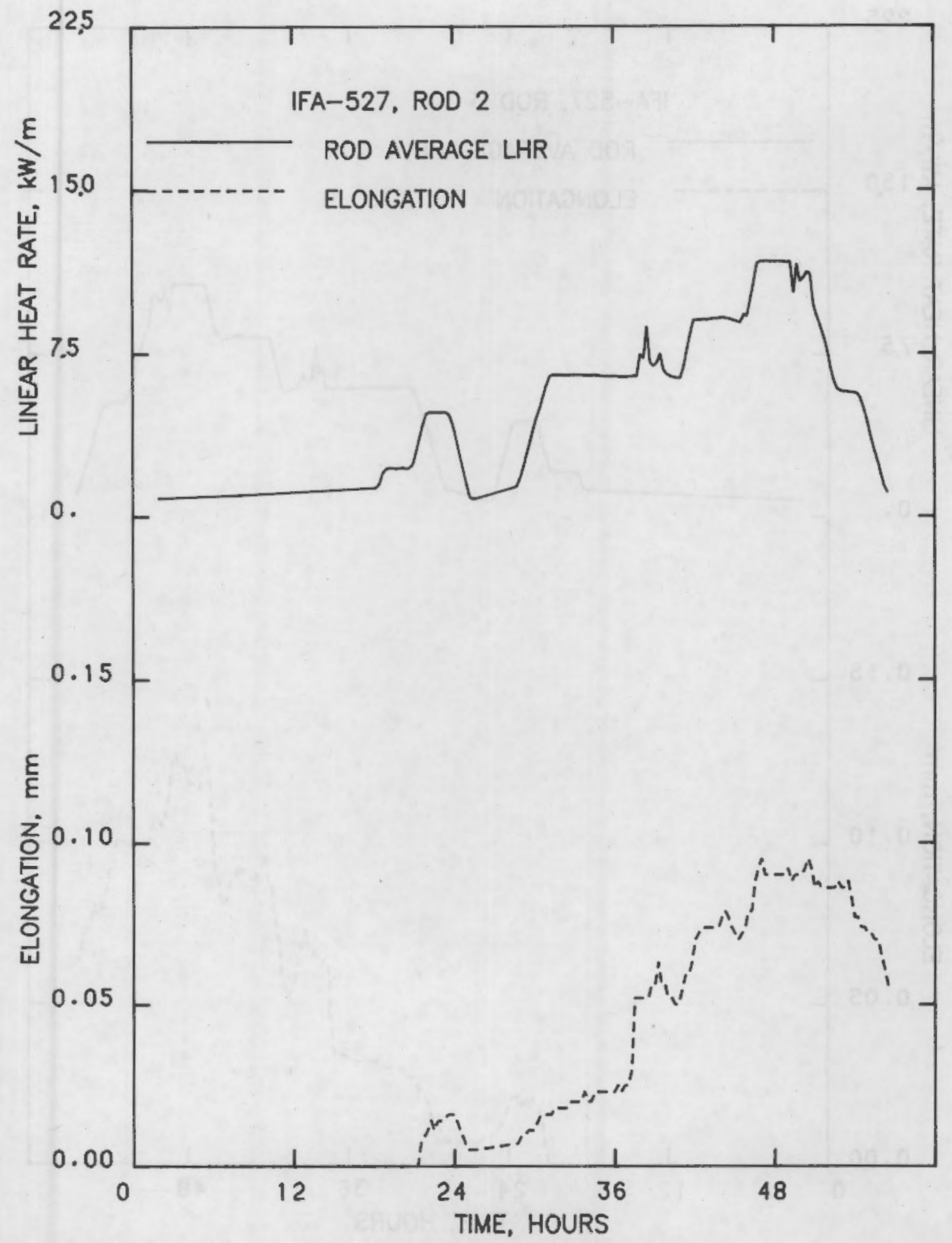

FIGURE 28. Fuel Rod Elongation and Average Linear Heat Rating for Rod 2 During the First Rise to Power 


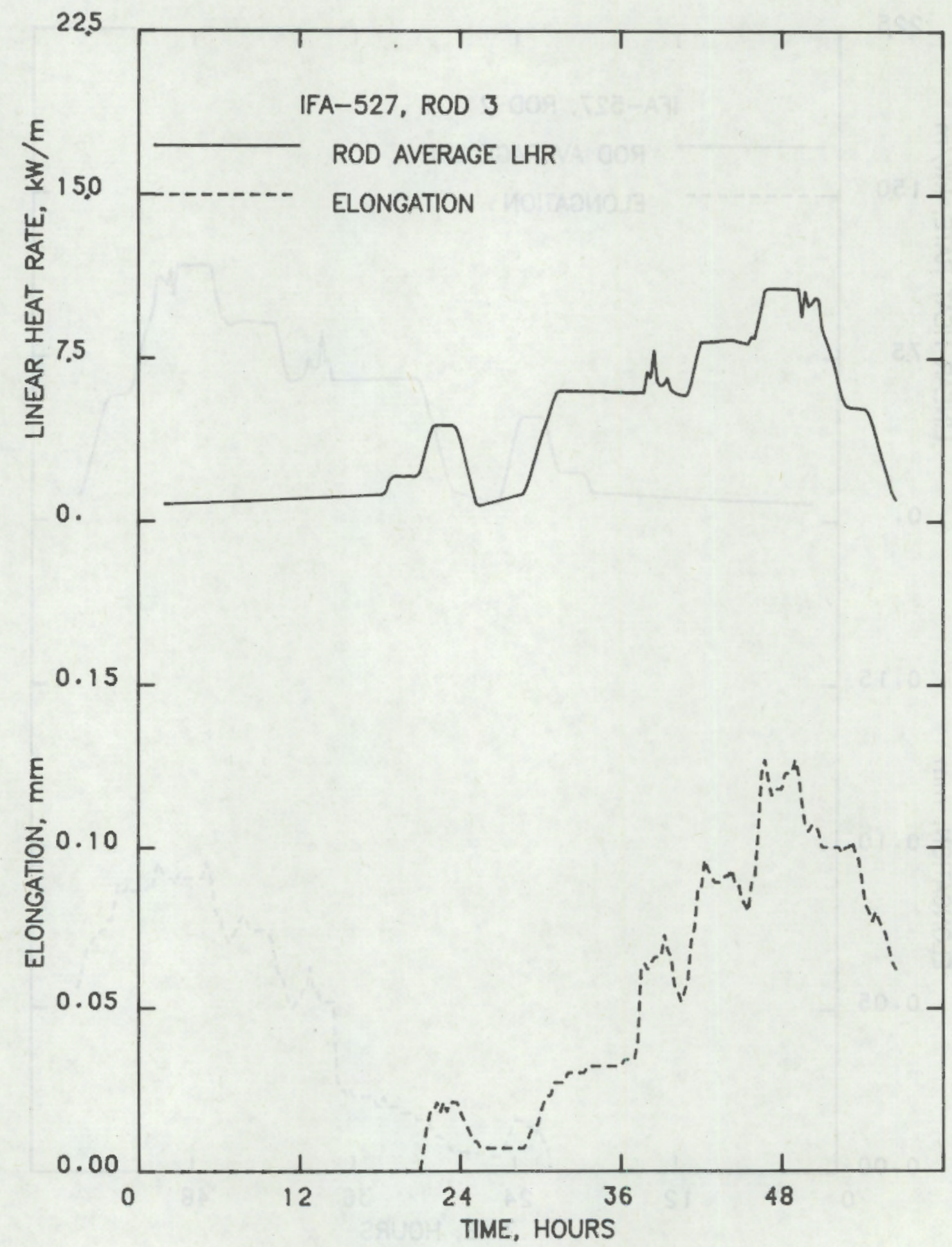

FIGURE 29. Fuel Rod Elongation and Average Linear Heat Rating for Rod 3 During the First Rise to Power 


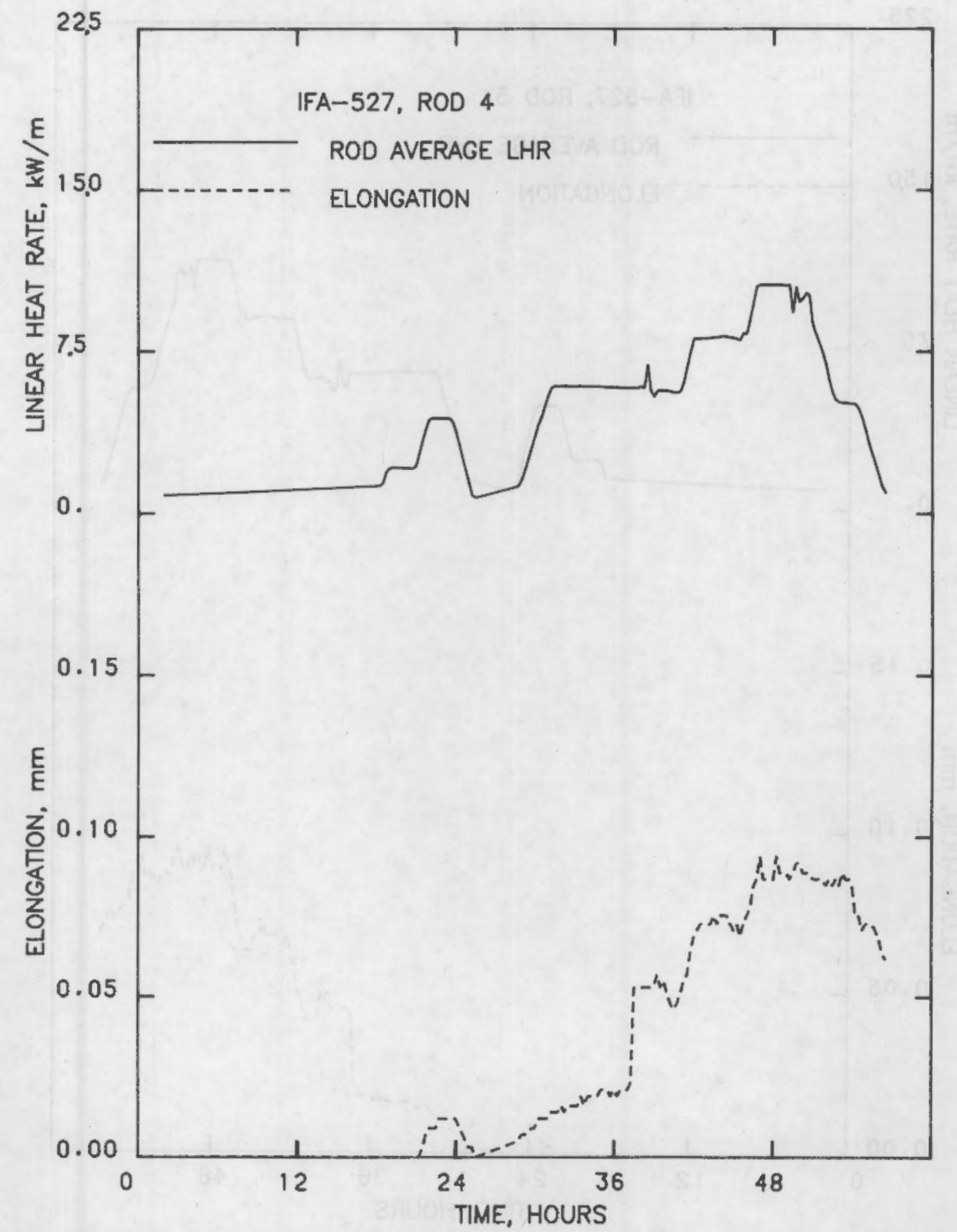

FIGURE 30. Fuel Rod Elongation and Average Linear Heat Rating for Rod 4 During the First Rise to Power 


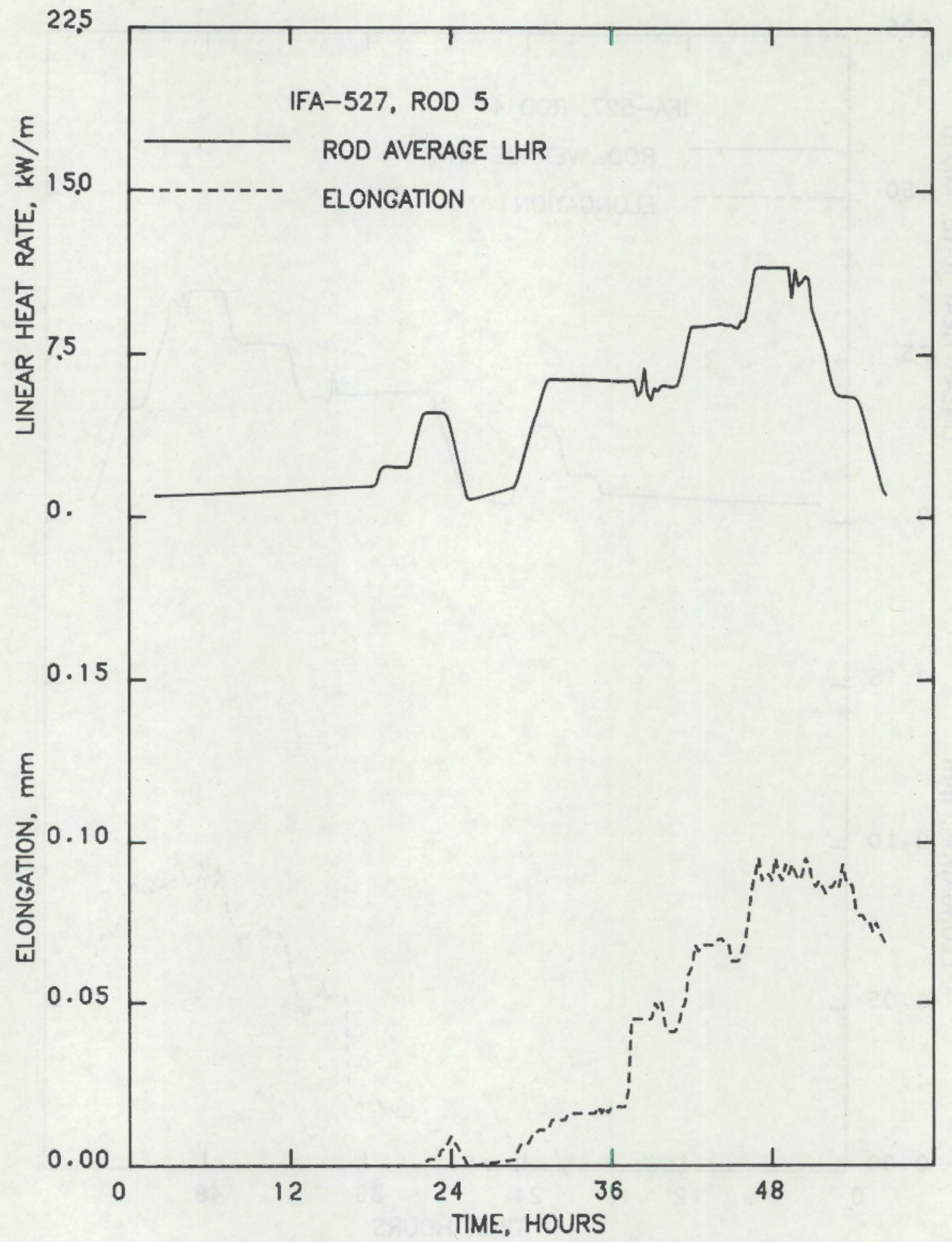

FIGURE 31. Fuel Rod Elongation and Average Linear Heat Rating for Rod 5 During the First Rise to Power 


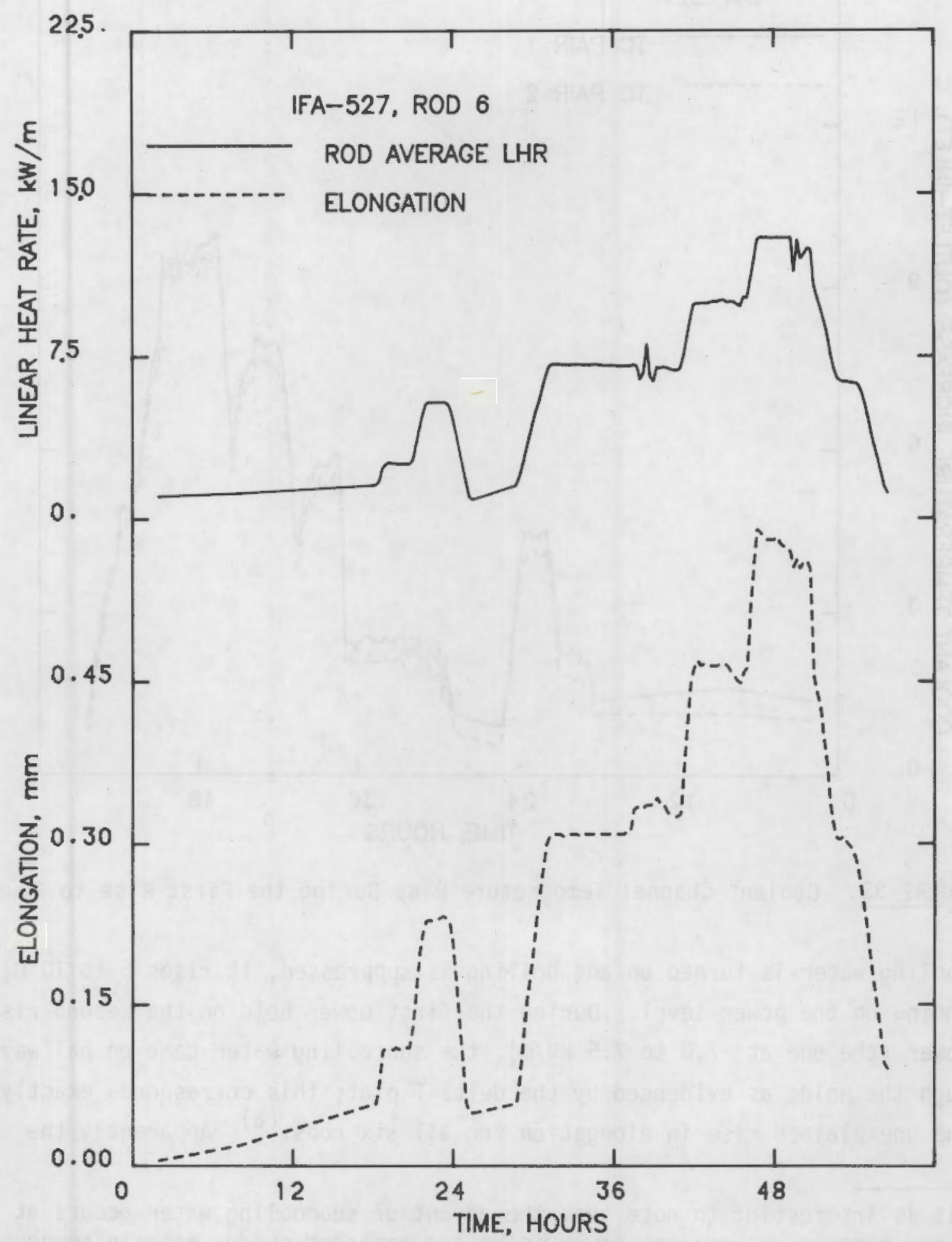

FIGURE 32. Fuel Rod Elongation and Average Linear Heat Rating for Rod 6 During the First Rise to Power 


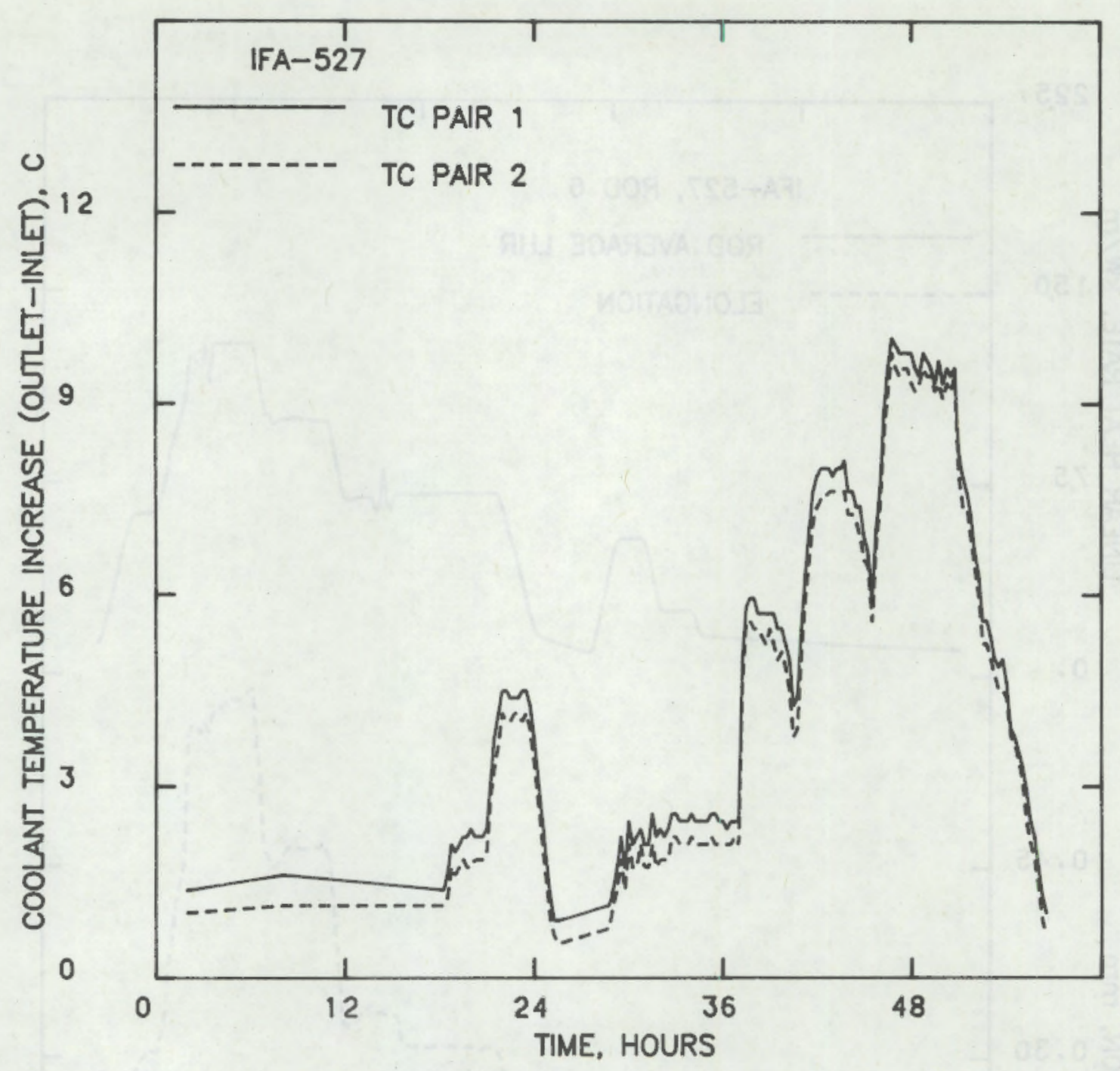

FIGURE 33. Coolant Channel Temperature Rise During the First Rise to Power

subcooling water is turned on and boiling is suppressed, it rises 5 to $10^{\circ} \mathrm{C}$, depending on the power level. During the first power hold on the second rise to power (the one at $\sim 7.0$ to $7.5 \mathrm{~kW} / \mathrm{m}$ ), the subcooling water came on halfway through the hold, as evidenced by the delta-T plot; this corresponds exactly to the unexplained rise in elongation for all six rods. (a) Apparently the

(a) It is interesting to note that the advent of subcooling water occurs at the same time as an apparent power spike and apparent sudden rise in temperature registered by some but not all fuel centerline thermocouples in the nominal-gap rods (see Figures 5 through 10). No explanation has been found for this. 
subcooling stayed on for the rest of the power ramp and biased the rest of the elongation data from that ramp. The exact amount of the bias is unclear, but it is in the positive direction in the range from 25 to $60 \mu \mathrm{m}$, depending on the rod and the power level. It is therefore concluded that elongation behavior for this first period of operation is significantly biased by subcooling effects.

The second feature--the $60-\mu m$ offset after first rise to full power--may, in fact, represent permanent cladding elongation aithough the same offset is observed for rod 6 (strong $P C I)^{(a)}$ as for rods 1 through 5 (weak PCI). Behavior During First Operating Period

During the first two months of operation subcooling did not affect these data as shown in Figure 34, which plots coolant delta-T versus time. Note that only in the first four days (the period previously discussed) does the coolant delta-T exceed the 1 to $3^{\circ} \mathrm{C}$ that is indicative of natural circulation.

The relative elongation (above the zero-power offset) for rods 1 and 6 during the second rise to full power is plotted against the rod-averaged power in Figure 35. The calculated cladding tube thermal expansion as estimated from MWRAM-calculated cladding temperatures and the axial Zircaloy tube expansion formula in MATPRO-10 (Reymann 1978) is also shown. Rod 6 shows immediate and significant PCI as evidenced by cladding elongation $f a r$ above that explained by thermal expansion alone, even at powers below $6 \mathrm{~kW} / \mathrm{m}$. It is significant that rod 1 (representative of rods 1 through 5) also shows some evidence of PCI at powers above about $9 \mathrm{~kW} / \mathrm{m}$. This trend continues for rods 1 through 5 for the first two months of operation (see Figures 36 through 38 ).

The offset at zero power indicated by Figures 36 through 38 seems to be increasing from 60 to over $100 \mathrm{um}$. This trend is even more evident when all the elongation data are plotted as a function of time (see Figures 39 through 44). It is also evident that the zero-power/full-power elongation difference for all rods attenuates as a function of time; the absolute magnitude of this reduction is most dramatic-for rod 6 . This may be due to the

(a) pellet-cladding interaction. 


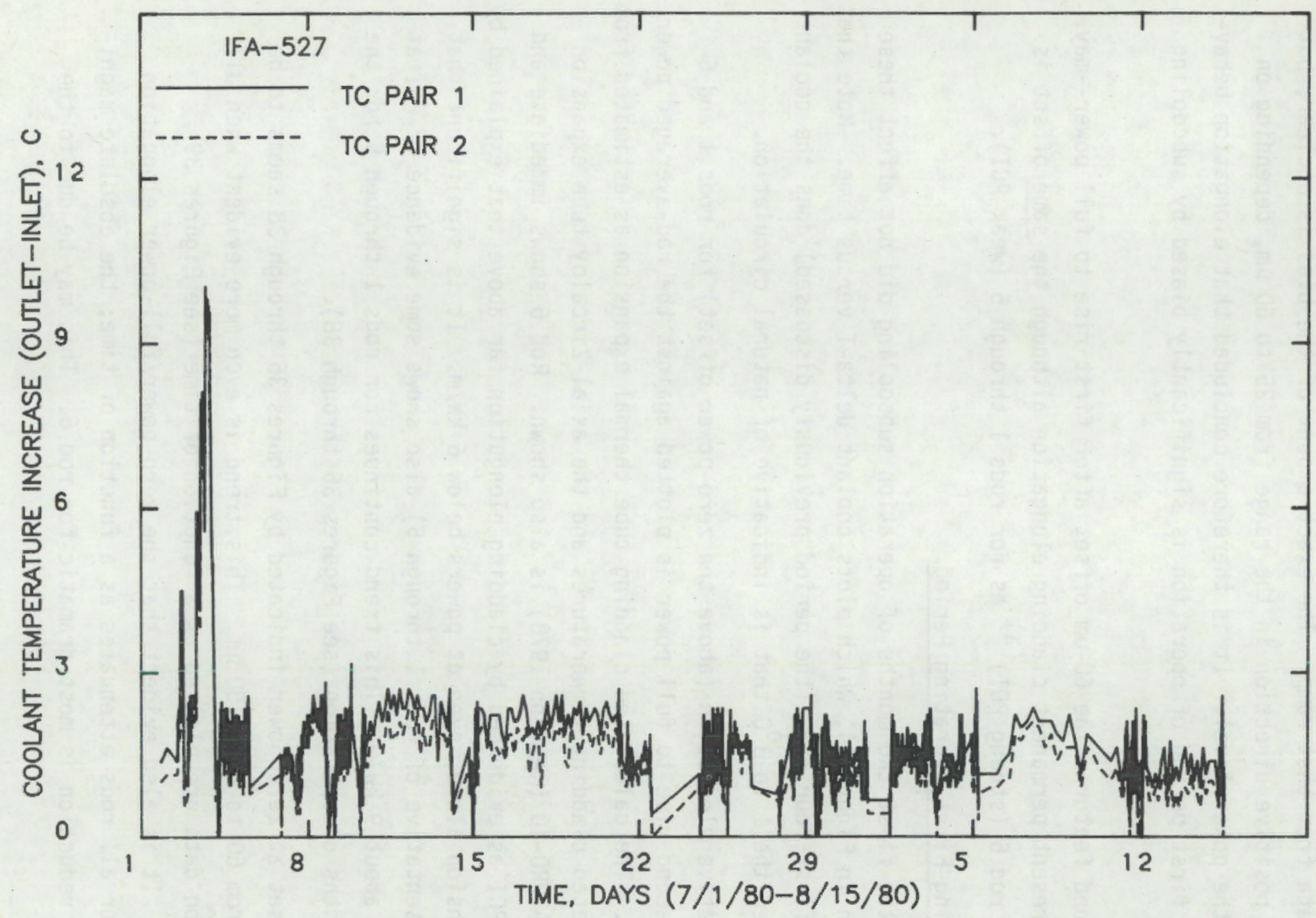

FIGURE 34. Coolant Channel Temperature Rise During the First Operating Period 


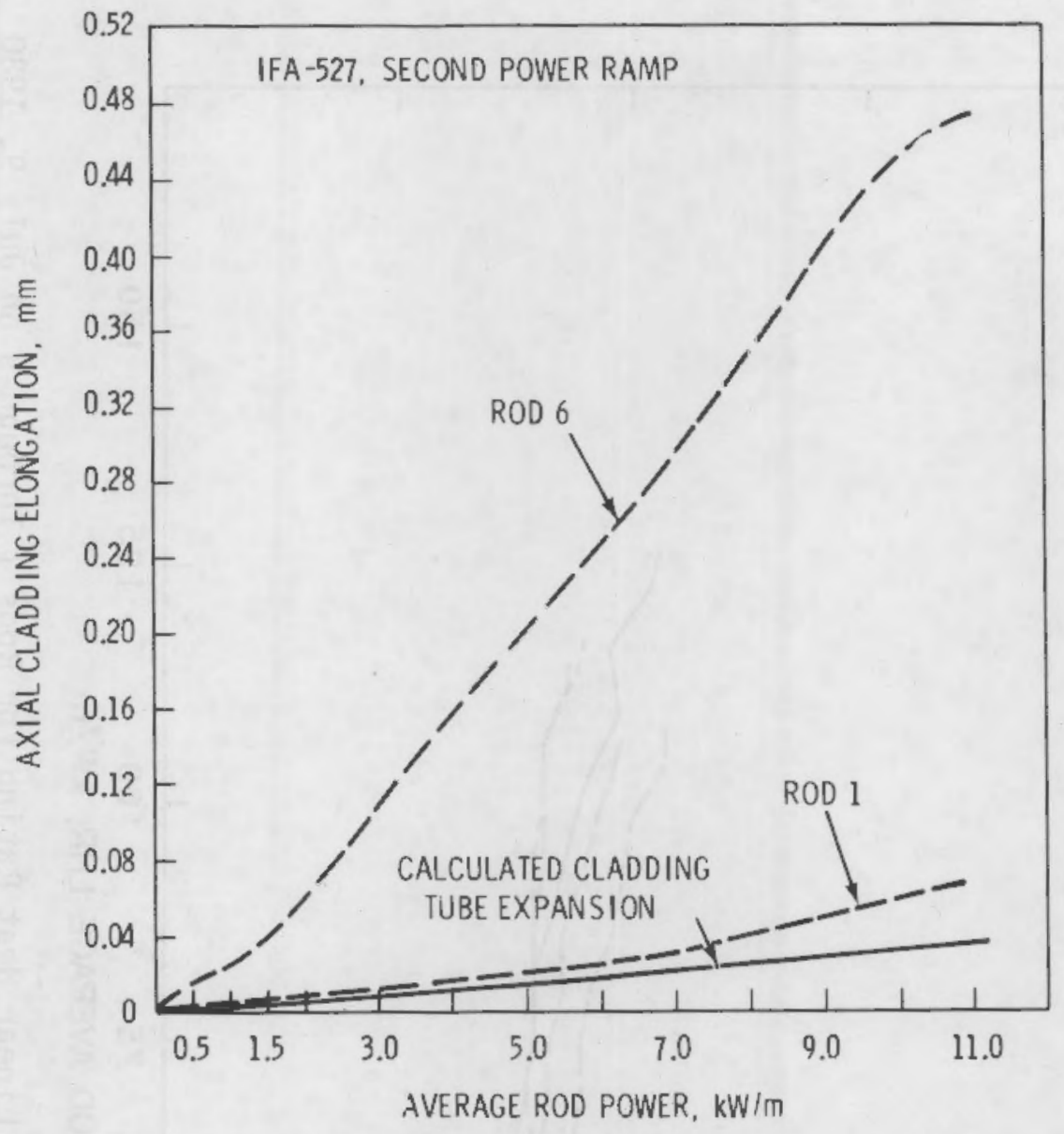

FIGURE 35. Rod Elongation Versus Average Linear Heat Rating During Second Rise to Power for Rods 1 and 6

formation of small, semipermanent axial gaps in the cracked fuel column that must close before further axial stress can apply to the cladding from the fuel column. The fact that the coolant (inlet) temperature dropped from 240 to $230^{\circ} \mathrm{C}$ on July 27 and continued at the lower value until the end of the period may also be influencing the data. 


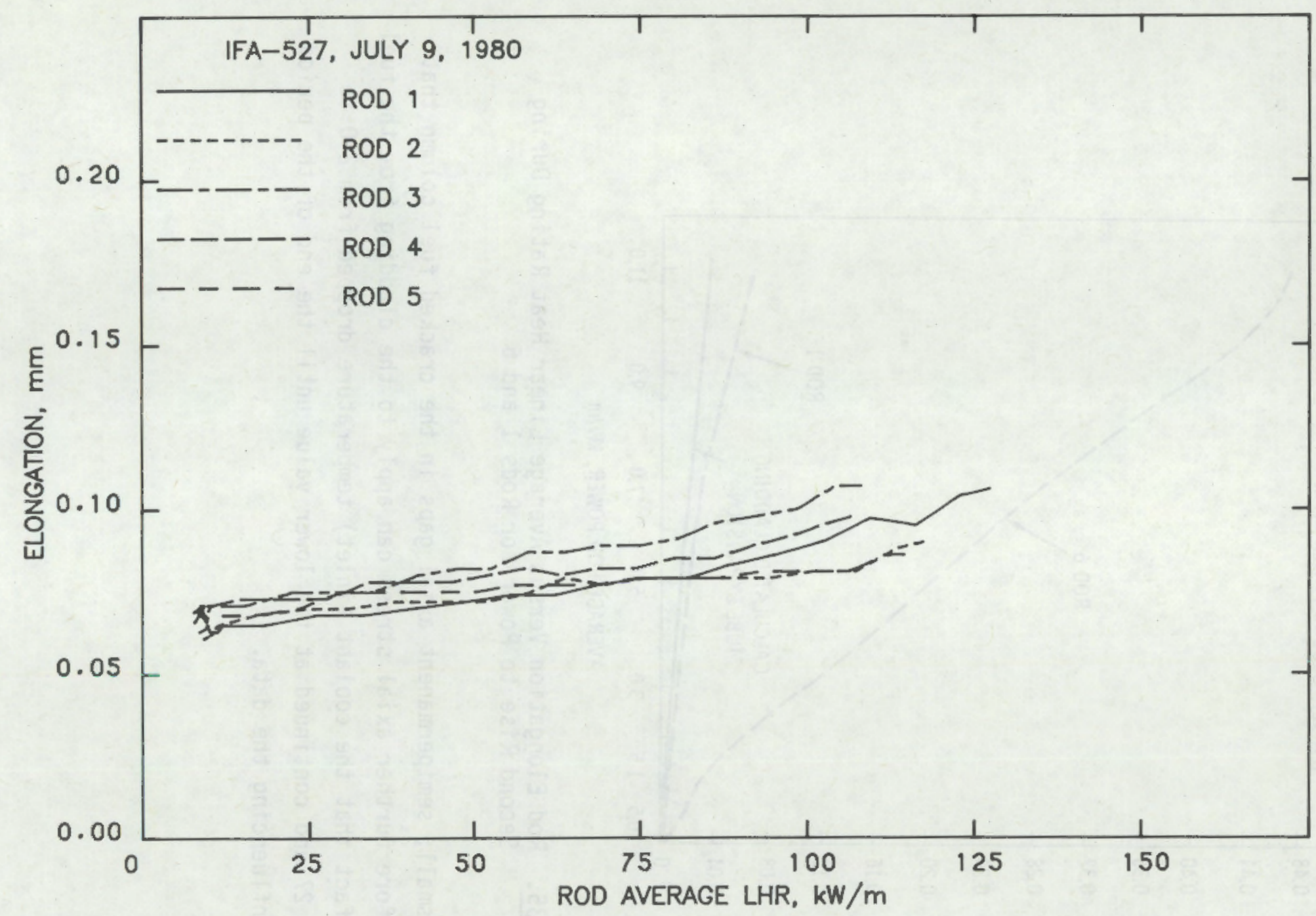

FIGURE 36. Rod Elongation Versus Average Linear Heat Rating for Rods 1 Through 5 on July 9, 1980 


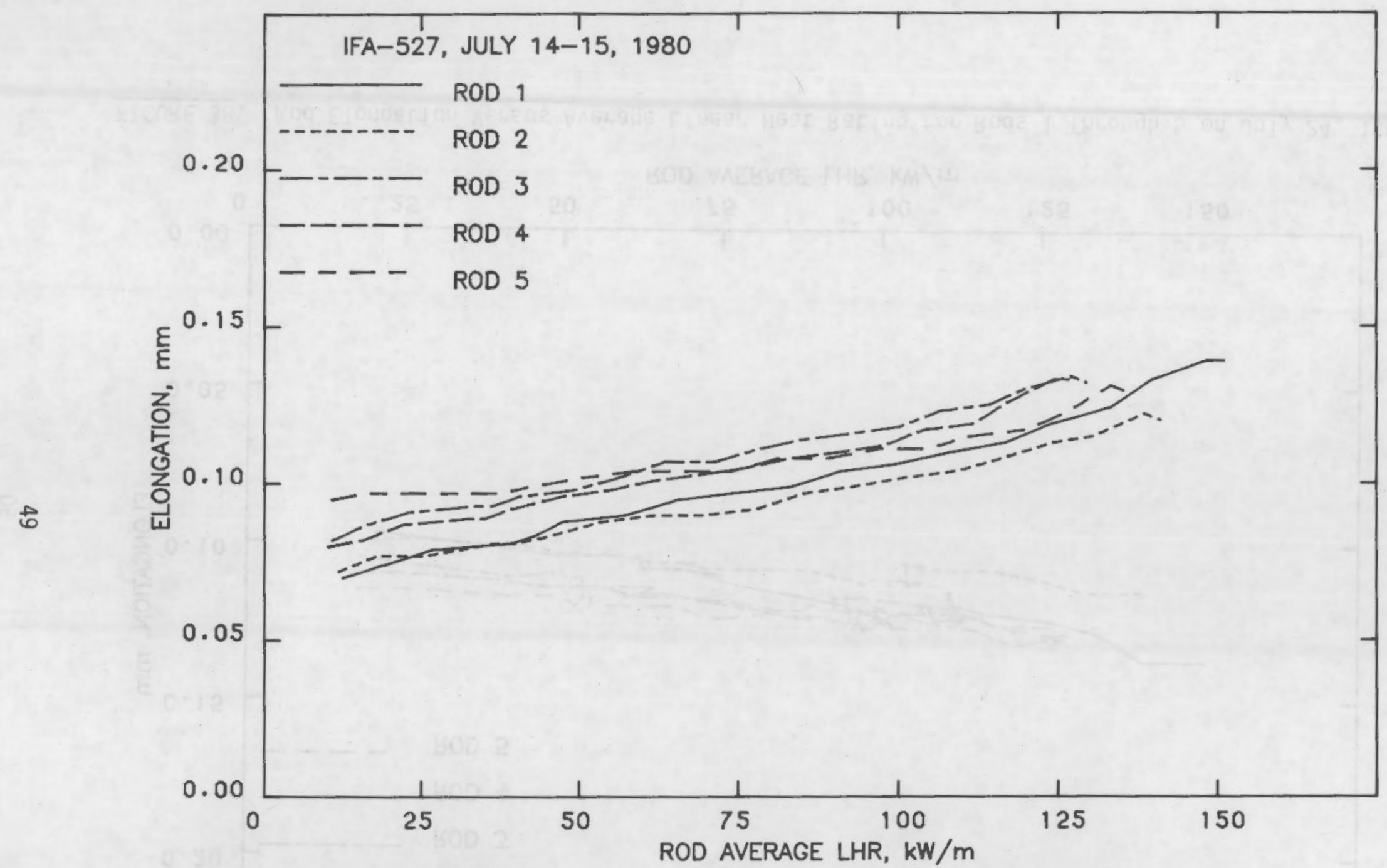

FIGURE 37. Rod Elongation Versus Average Linear Heat Rating for Rods 1 Through 5 on July 14 and 15, 1980 


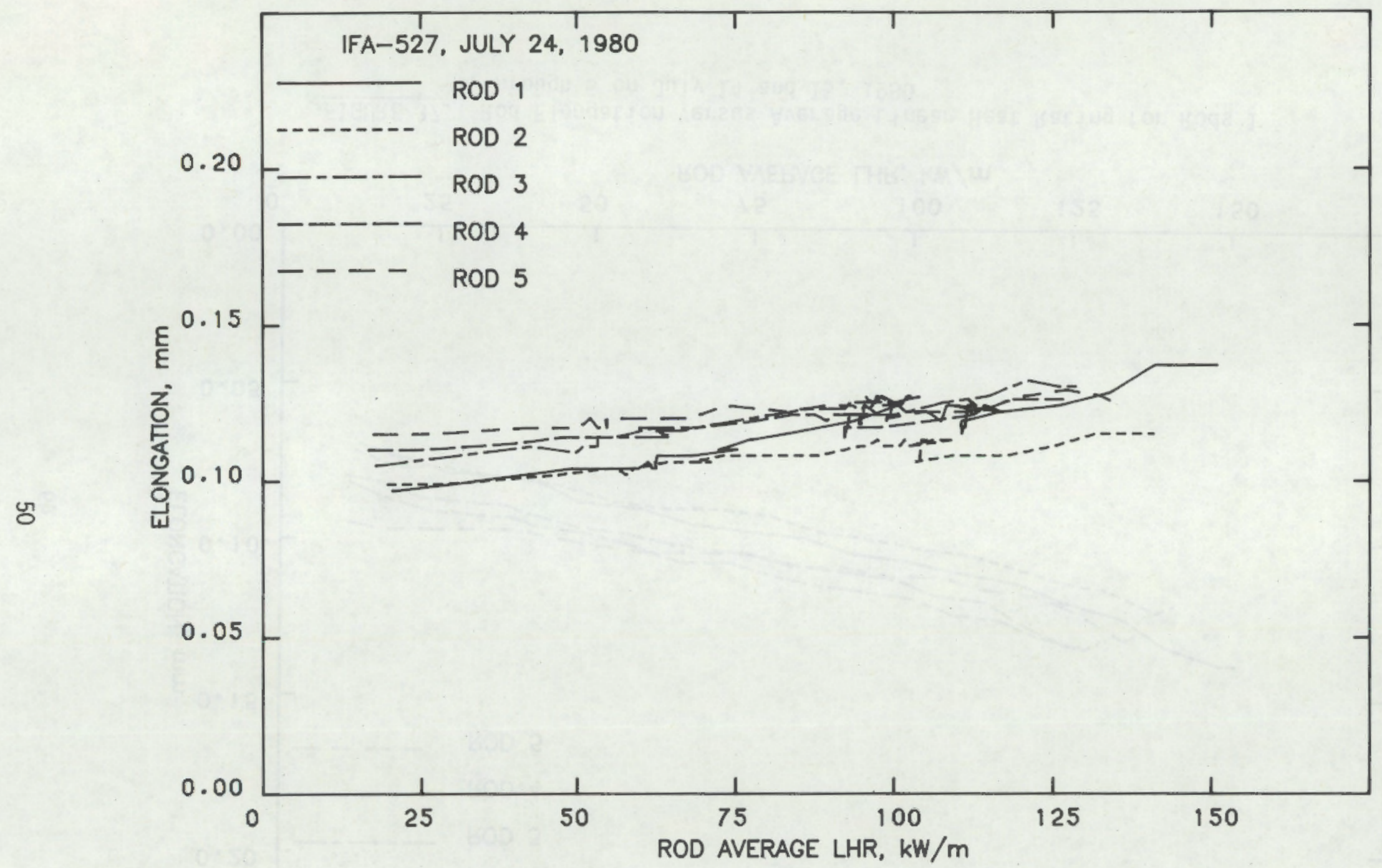

FIGURE 38. Rod Elongation Versus Average Linear Heat Rating for Rods 1 Through 5 on July 24, 1980 


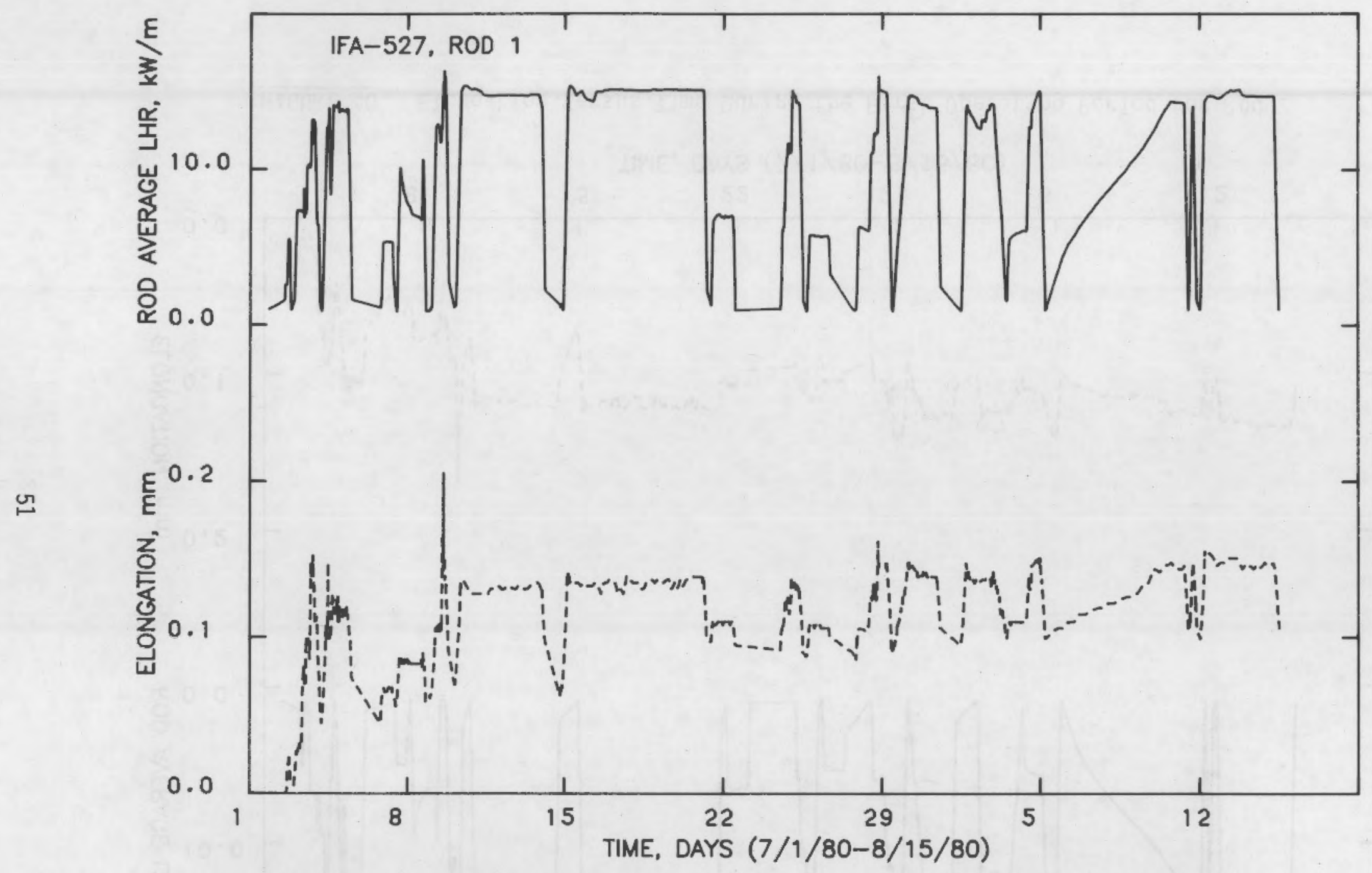

FIGURE 39. Elongation Versus Time During the First Operating Period for Rod 1 


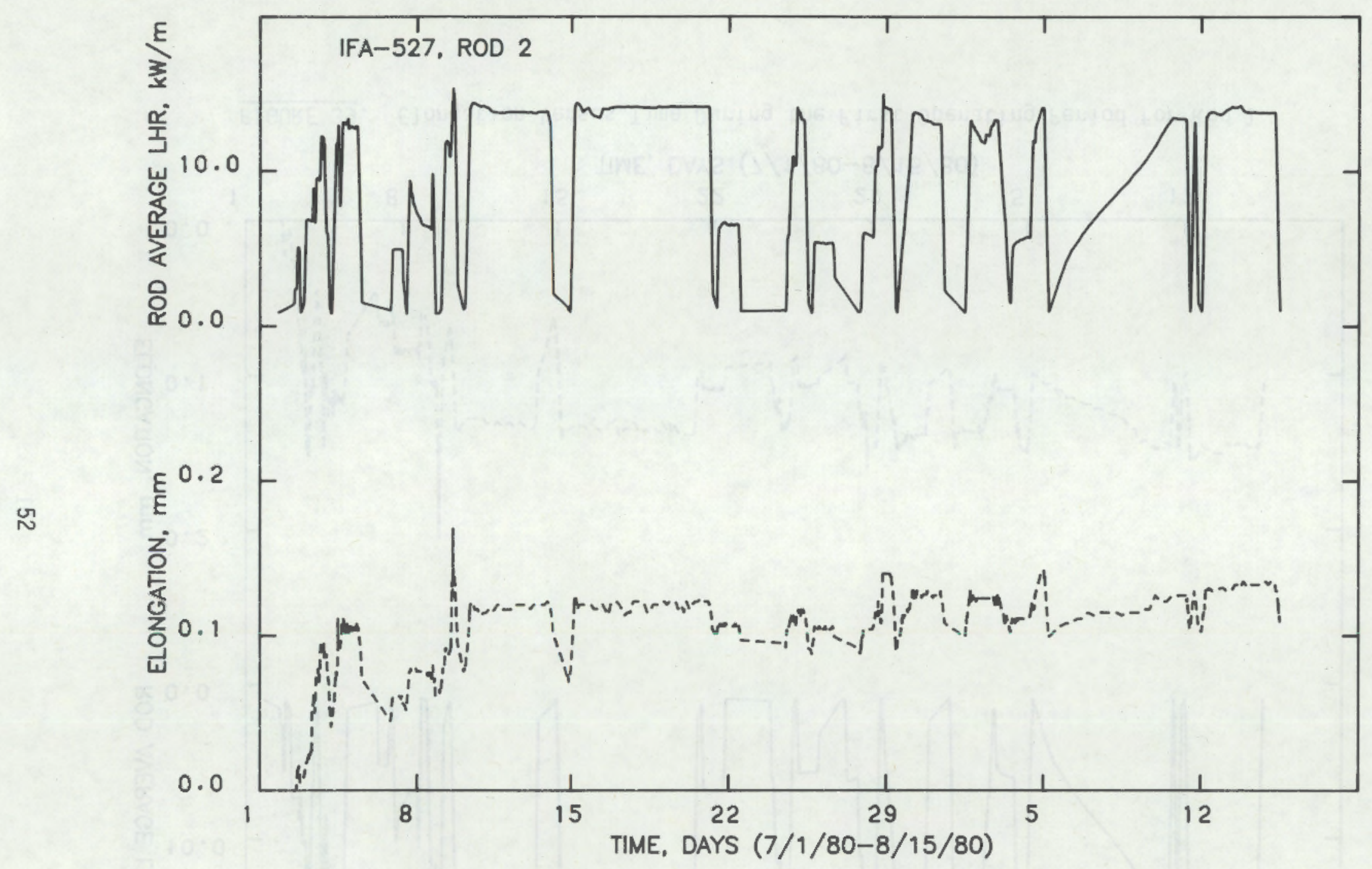

FIGURE 40. Elongation Versus Time During the First Operating Period for Rod 2 


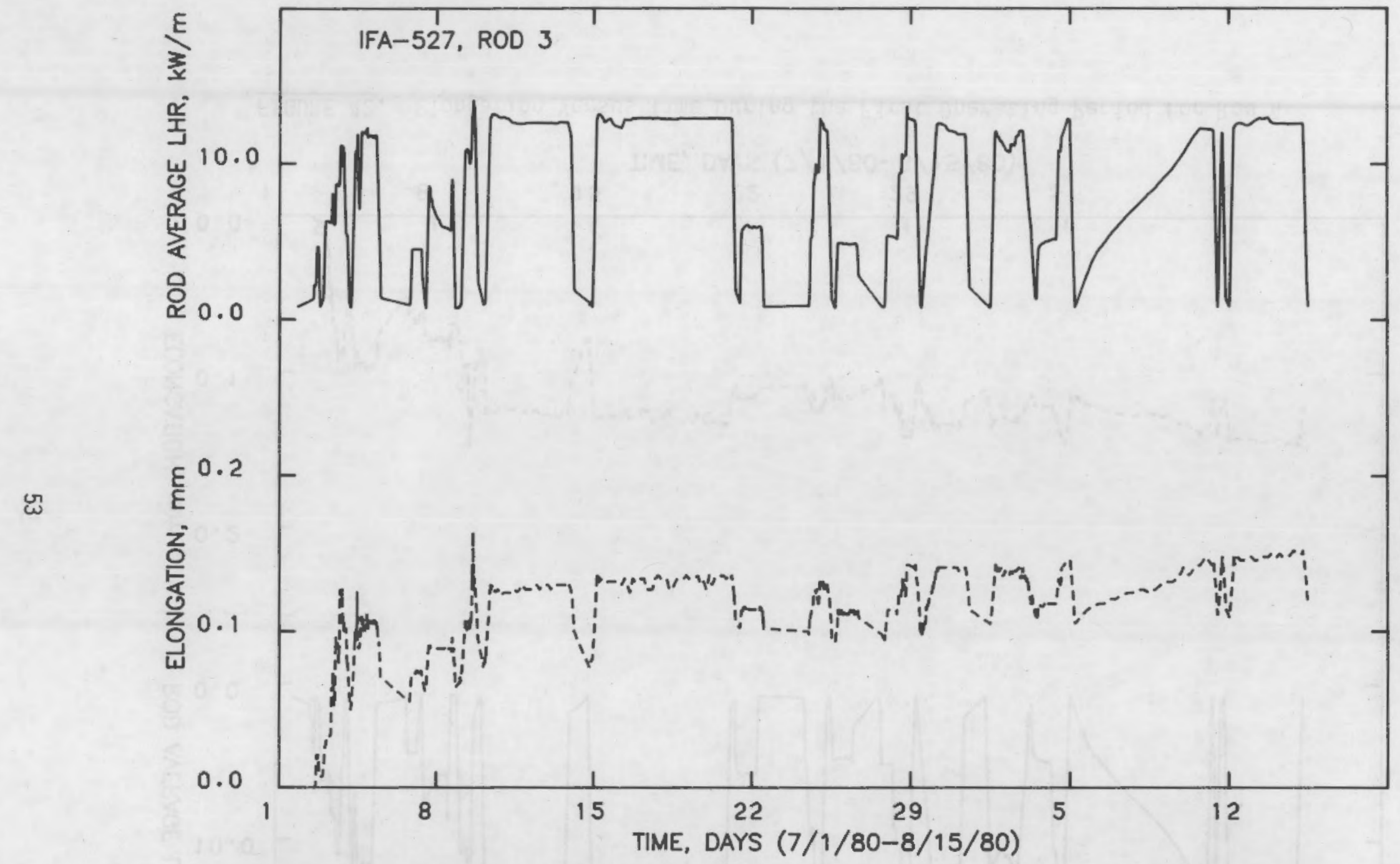

FIGURE 41. Elongation Versus Time During the First Operating Period for Rod 3 


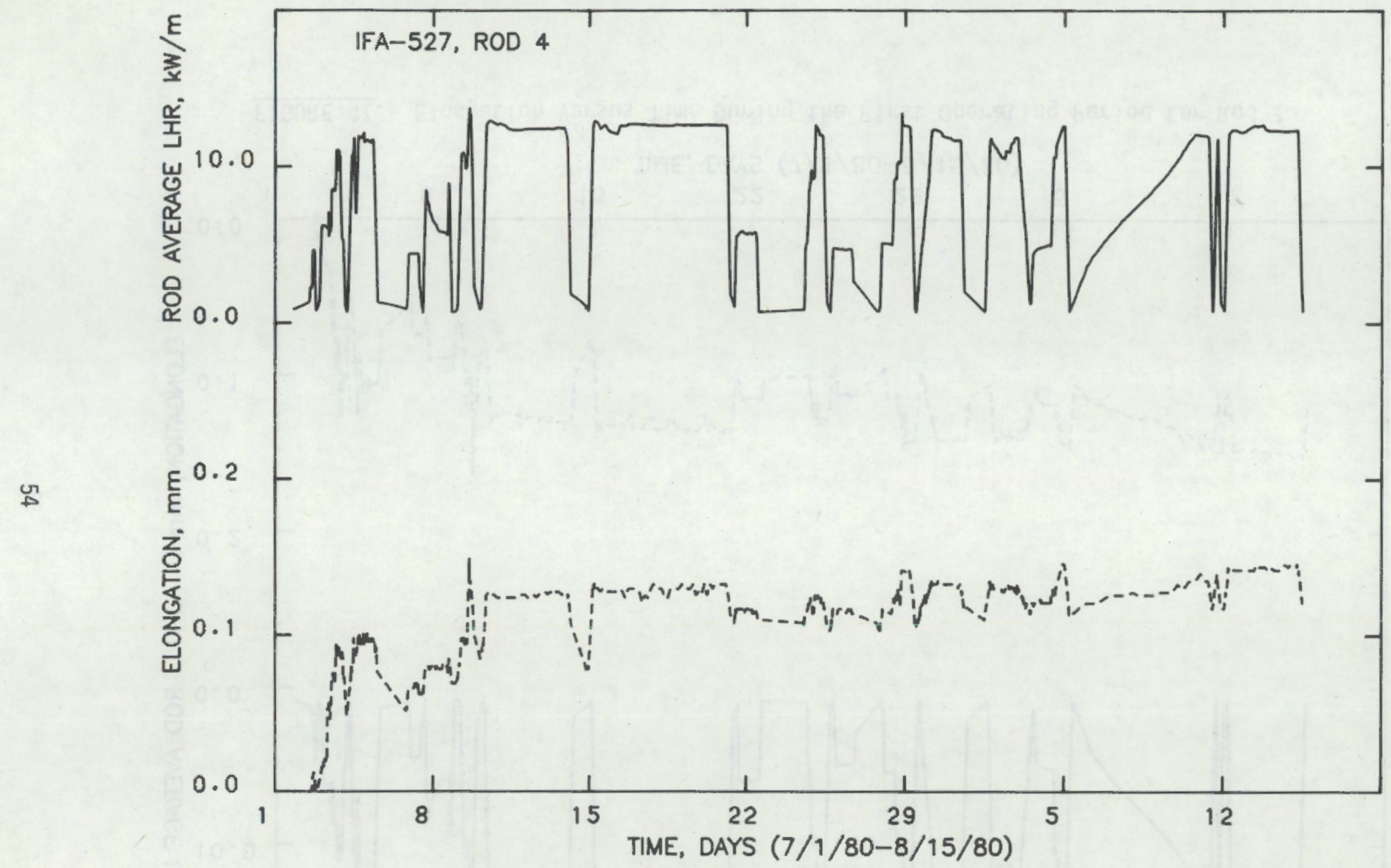

FIGURE 42. Elongation Versus Time During the First Operating Period for Rod 4 


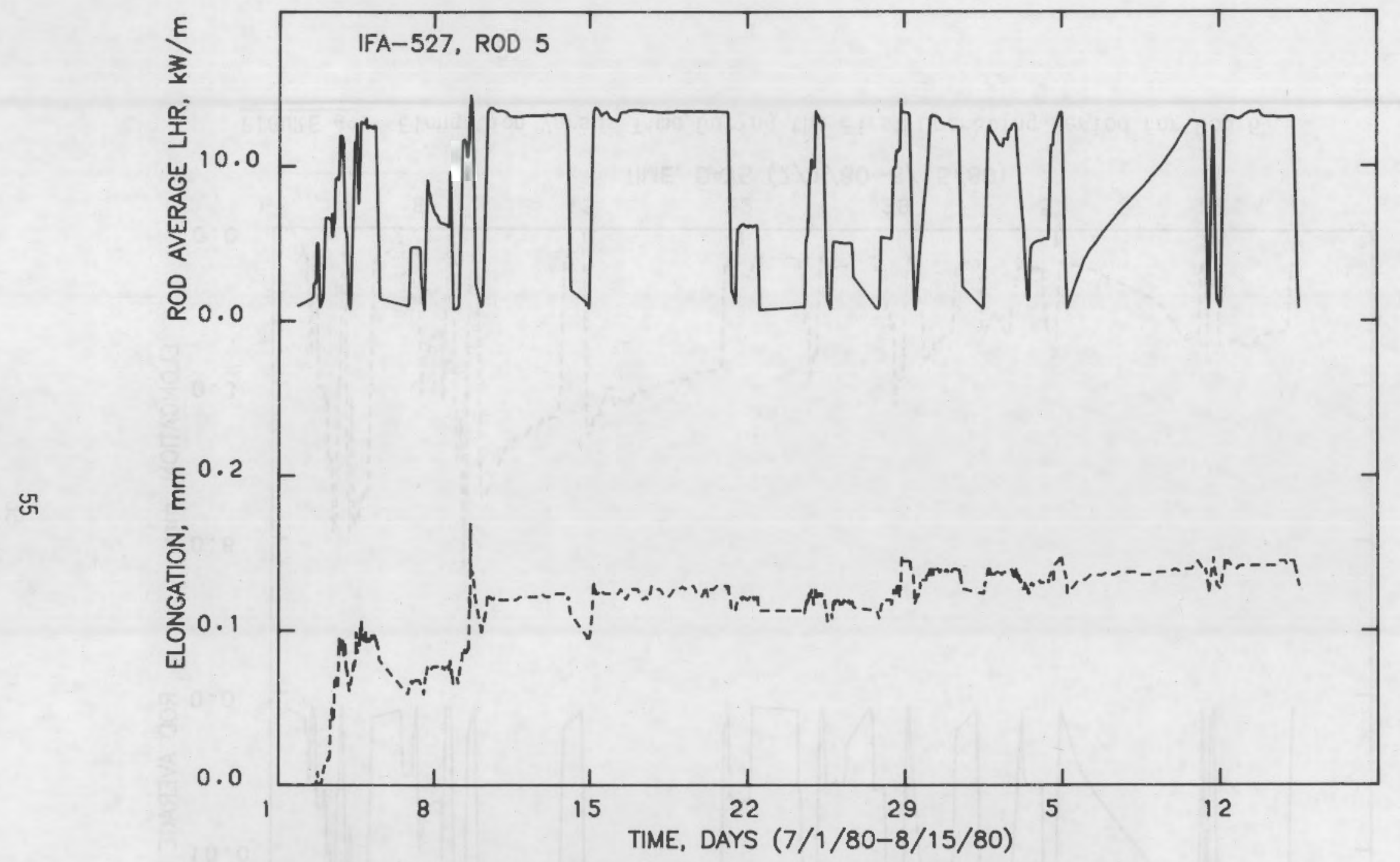

FIGURE 43. Elongation Versus Time Ouring the First Operating Period for Rod 5 


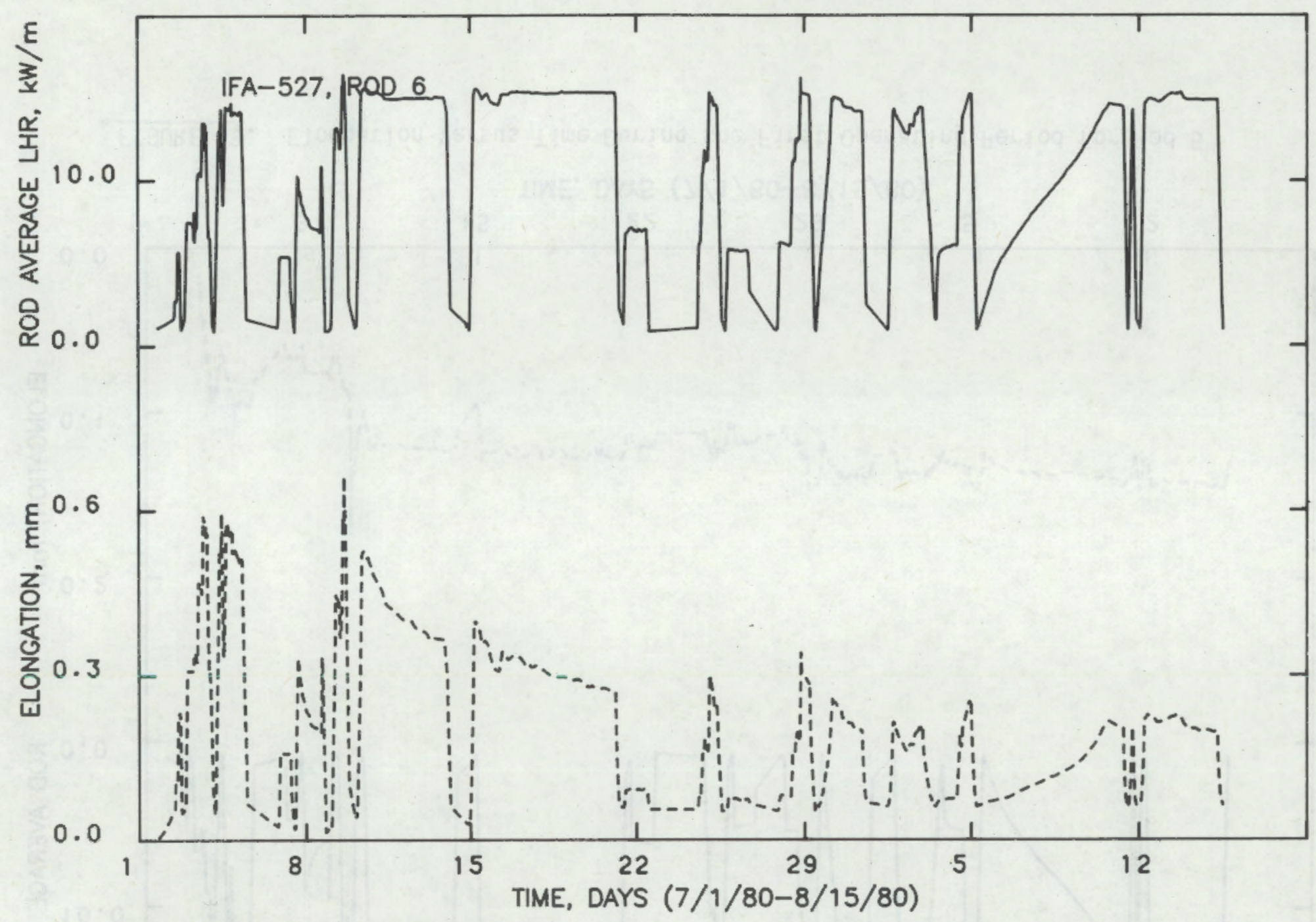

FIGURE 44. Elongation Versus Time During the First Operating Period for Rod 6 
ROD GAS PRESSURE DATA

The gas pressure histories for rods 1 through 5 are very similar although this aggregate behavior departs significantly from that of IFA-513. Unfortunately, the failure of the pressure transducer for rod 6 upon first startup precludes a gas pressure history comparison between large-gap and small-gap rods. All rods were initially filled with xenon gas at 0.1-MPa (one atmosphere) pressure at room temperature $\left(20^{\circ} \mathrm{C}\right)$.

Initial Ramp Data

Table 5 shows the probable distribution of the gas in the as-fabricated rods, and the typical behavior of relative gas pressure versus rod-averaged power during the first few ramps to power is shown in Figure 45. At low power and in the absence of severe fuel cracking, one would expect the same distribution of void volume; i.e., the majority of the gas would be at or near the coolant temperature of about $240^{\circ} \mathrm{C}$.

The BOL low-power pressure data certainly support the above expectation; the zero-power gas pressure is near $0.18 \mathrm{MPa}$ in every case--exactly the pressure expected from the Kelvin temperature ratio $(513 / 293)$. This is not surprising since the raw pressure data are "calibrated" by additive constants to achieve just that result.

TABLE 5. Estimated Void Volume Distribution for Rods 1 Through 5 of Instrumented Fuel Assembly (IFA) -527

\begin{tabular}{|c|c|c|}
\hline Item & $\begin{array}{c}\text { As-Fabricated } \\
\text { Void Volume, } \\
\mathrm{ml}\end{array}$ & $\begin{array}{l}\text { Estimated Void Volume } \\
\text { at Low Power }(1.0 \mathrm{~kW} / \mathrm{m}) \\
\text { After Operation, ml }\end{array}$ \\
\hline Plenum Plus Pressure Transducer & 5.5 & 5.5 \\
\hline Fue $1-C 1$ adding Gap & 2.9 & ? (down to 0.9 ) \\
\hline $\begin{array}{l}\text { Fuel Column Gaps } \\
\text { (rough pellet interfaces) }\end{array}$ & 0.5 & 0.5 \\
\hline Fuel Pellet Cracks & $\underline{0}$ & ? (up to 2.0 ) \\
\hline TOTAL & 8.9 & $\leq 8.9$ \\
\hline
\end{tabular}




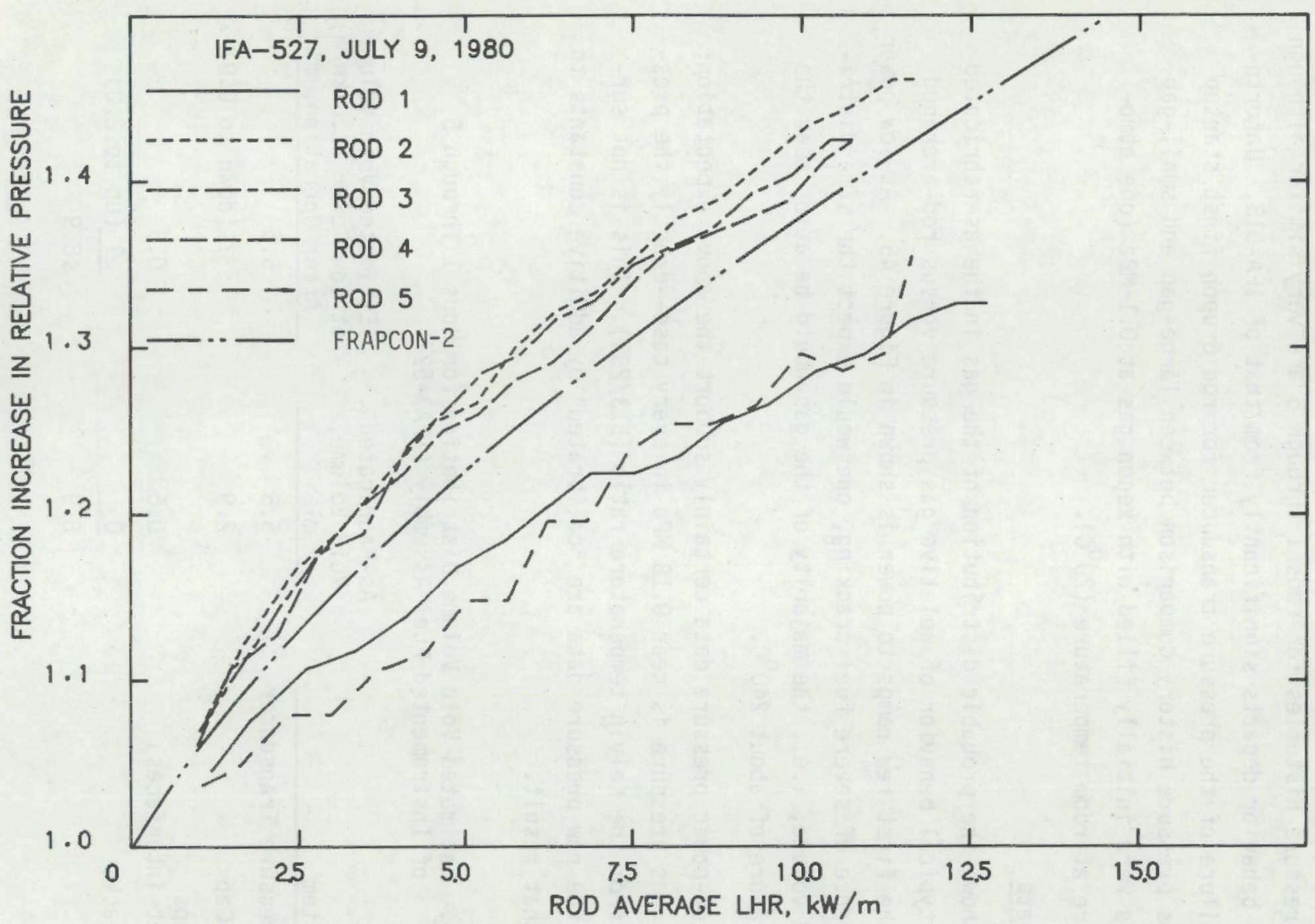

FIGURE 45. Relative Gas Pressure for Rods 1 Through 5 on First Rise to Power (divided by low-power value) 
To emphasize the reasonableness of the relative pressure increases indicated in Figure 45, the calculated increase from the FRAPCON-2 code (Berna 1980 ) is also shown, which accounts for fuel cracking, relocation, and consequent shift of gas from gap to cracks.

Behavior During First Operating Period

After the first few ramps, the low-power gas pressure shifts to higher values and finally settles at about $0.23 \mathrm{MPa}$. The full-power gas pressure values shift upward too but not so dramatically. It is not clear whether these small shifts are real or not; they are clearly noticeable in Figure 46 where all pressure data versus time for rods 1 through 5 during the July/August operating period are shown. This shift does not occur suddenly on July 27 , when the coolant temperature underwent a sudden and permanent drop to $230^{\circ} \mathrm{C}$; nevertheless, the later shifts may be enhanced by that change in coolant temperature. 


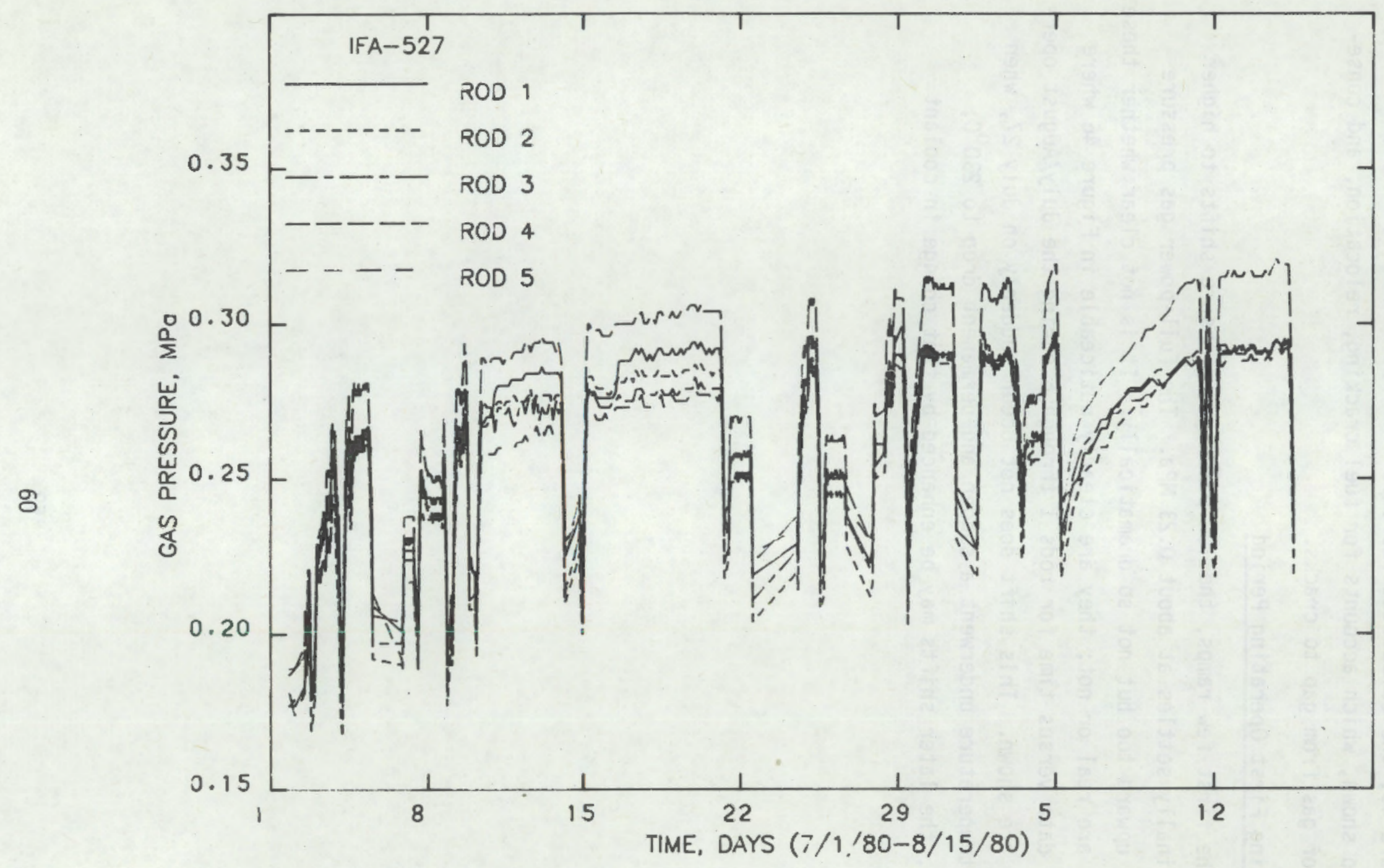

FIGURE 46. Fuel Rod Gas Pressure for Rods 1 Through 5 During the First Operating Period 
BEHAVIOR DURING AND AFTER ROD FAILURE

(September 10 to October 4,1980 )

The reactor remained on cold shutdown from about August 15-17 to September 10, 1980. During this time, coolant temperatures were allowed to fall to below $70^{\circ} \mathrm{C}$. Before the subsequent startup, rods 1,4 , and 5 definitely failed as deduced from very high internal gas pressure readings (approaching the system pressure of $3.4 \mathrm{MPa}$ ) and very low fuel temperatures (commensurate with steam replacing the xenon fill gas). Figures 47 and 48 surmarize the behavior of the IFA-527 rods from September 10 to October 4 . Figure 47 shows the pressure behavior of all five rods; and Figure 48 shows the temperature behavior of rods 1,2 , and 3 . Temperatures for rods 4 and 5 , which are extremely close to those for rod 1, are not shown.

Even before the assembly began to produce power, the pressure in the failed rods ( 1,4 , and 5$)$ rose with the increasing coolant pressure, indicating that pressure boundary failure occurred before the power ramp (see figure 47 ). It is speculated that annealing and relaxation of the 0-ring seals at the ends of the rods caused the failure, which could well have happened when the coolant temperature first fell significantly below $227^{\circ} \mathrm{C}$ (about August 20).

Although the pressure in rod 2 began to rise along with the system pressure, it subsequently dropped back to $0.26 \mathrm{MPa}$, representative of the apparently unfailed rod 3--indicating, perhaps, a resealing of the rod. Such pressure spikes on startup were observed in a similarly designed IFA-513 rod that subsequently failed (Edler 1980), probably for the same reasons as the IFA-527 rods. Rods 4 and 5 appeared to reseal and lose some pressure, probabiy due to the chemical reaction of steam with fuel and cladding; but their water vapor inventory was apparently large enough that this did not significantly affect their fuel temperatures.

The temperature histories in $\mathrm{Figure} 4 \mathrm{~B}$ match the pressure histories very well. Rod 3 (unfailed) fuel temperatures climb back to the levels experienced in August. Rod 2 (resealing) appears to lose its steam fill (probably by hydration) so that in a few days its temperatures climb back to the level of rod 3 (at first they agreed with rod 1 temperatures). Finally, rod 1 


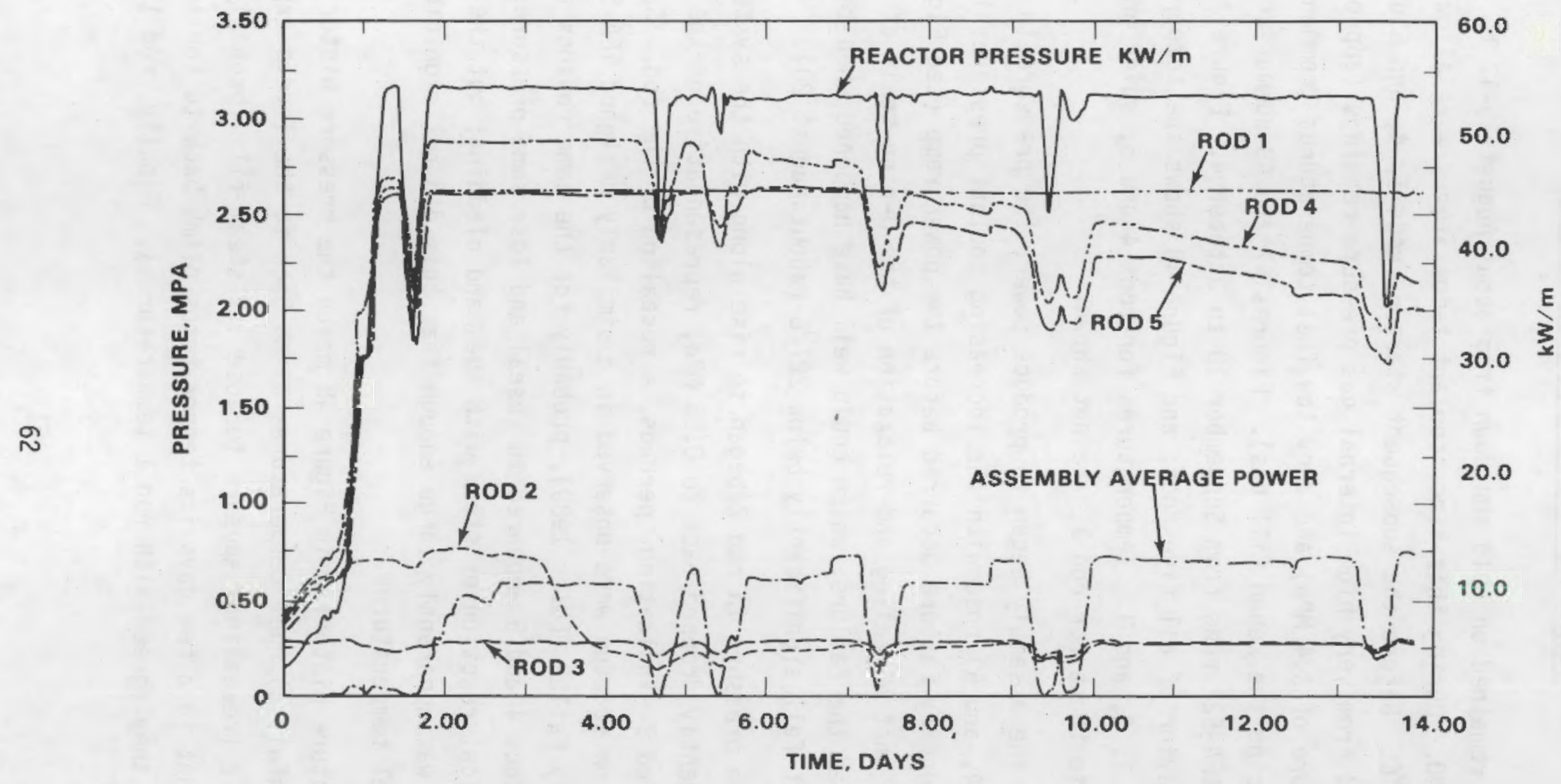

FIGURE 47. Gas Pressure Behavior of Rods 1 Through 5 During the September 10-12, 1980, Startup 


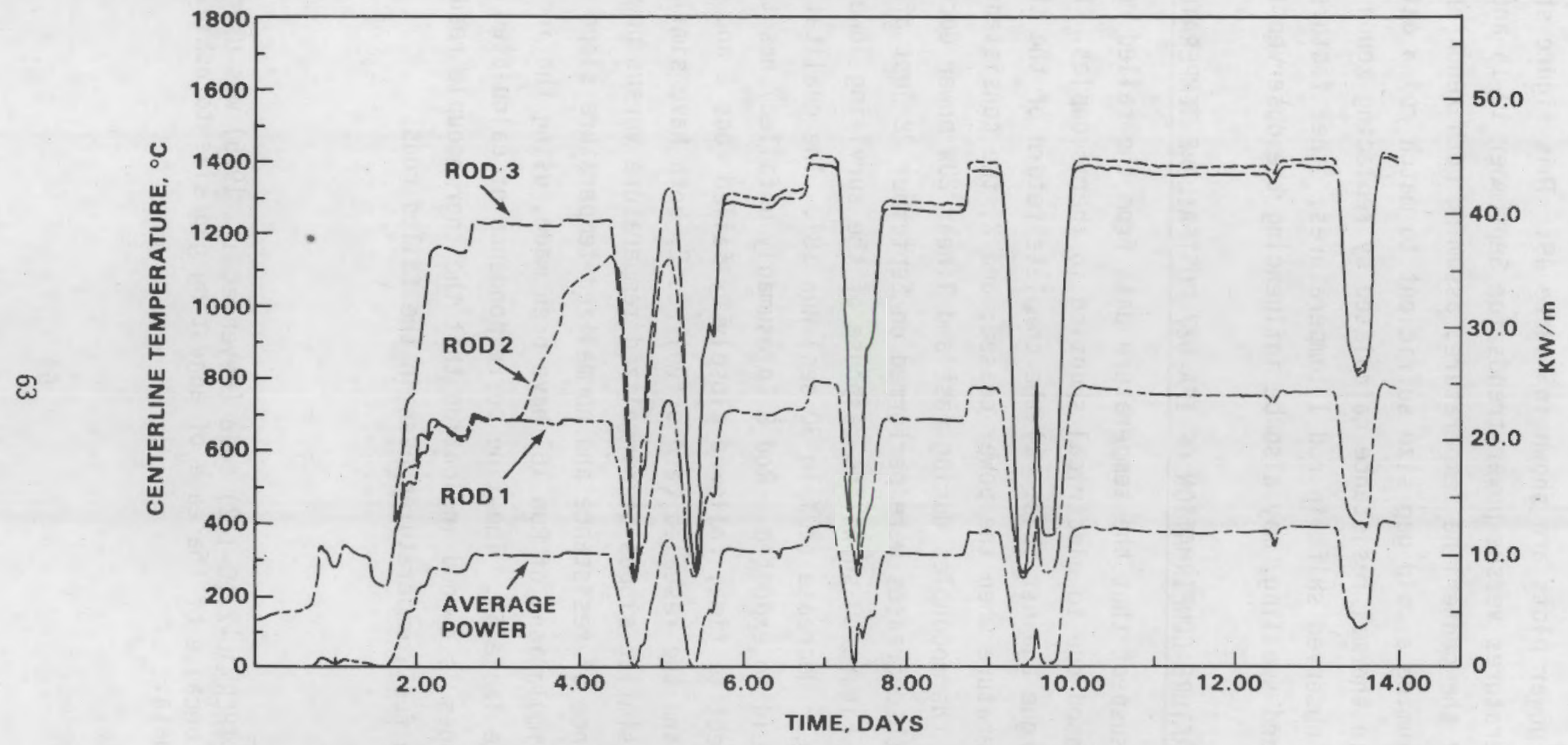

FIGURE 48. Lower Fuel Centerline Temperatures for Rods 1, 2, and 3 During the September 10-12, 1980, Startup 
temperatures remain extremely low compared to August levels. To emphasize that rod 1 temperatures are consistent with steam replacing xenon as fill gas, temperature versus power plots are shown in Figure 49. This figure shows rod 1 and 3 fuel temperatures versus power trends for September 10-15 and GAPCON-2 (a) calculations for the centerline temperature, assuming both xenon and steam as fill gas and assuming a cold gap size sufficient to match rod 3 data. The relative shift in thermal resistance calculated by replacing xenon with steam is equal to the observed shift in rod 1 temperatures; other factors, such as fuel oxidation and swelling, may also be influencing the observed temperatures.

\section{TRANSIENT TEMPERATURE CONFIRMATION OF IFA-527 POSTFAILURE TEMPERATURE DATA}

One might suspect that the temperature data from the failed rods would be erroneous or biased due to electrical shunting in thermocouples. However, two facts seem to argue against this: 1) the complete return of the thermocouples to coolant temperature when the power ceased; and 2) the consistent transient response of the thermocouples during fast and linear $20 \%$ power decreases. Fortunately, such decreases were performed on September 22 just after the failures occurred. Figure 50 shows the response of the surviving lower thermocouples to the linear decrease (20\% in $30 \mathrm{sec})$ Run 187 . The qualitative behavior is just what would be expected. Rod 6 (presumably unfailed) has the steepest slope with respect to time, followed closely by failed rods 1 and 4 . The unfailed rod 3 and the resealed (and dried) rod 2, both have similar thermal resistance and similar slopes of normalized temperature versus power. To make the correspondence of resistance and normalized temperature slope more quantitative, MWRAM simulations of Run 187 have been made, using the indicated rod resistances (see Table 6). The fine correspondence of calculated and measured temperature slopes is a good indication that the thermocouple readings are reflecting true fuel temperatures even in the failed rods.

(a) The GAPCON-THERMAL-2 (G-T-2) code (Beyer et al. 1975) was used for this comparison because of the ease of adjusting gap size to achieve a match to xenon rod data. 


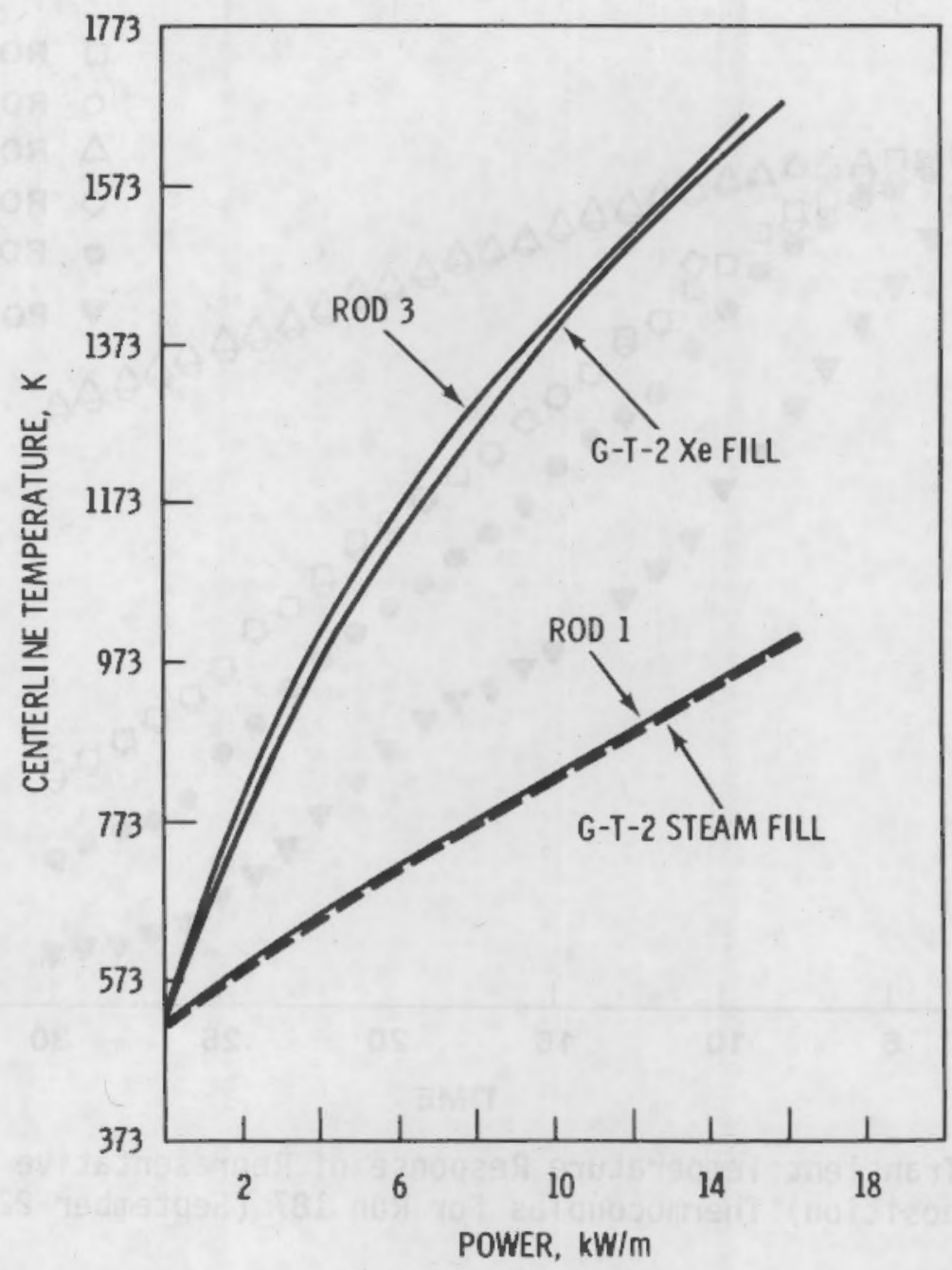

FIGURE 49. Measured and Calculated Centerline Temperatures for Rods 1 and 3 (failed and unfailed) in September 1980 


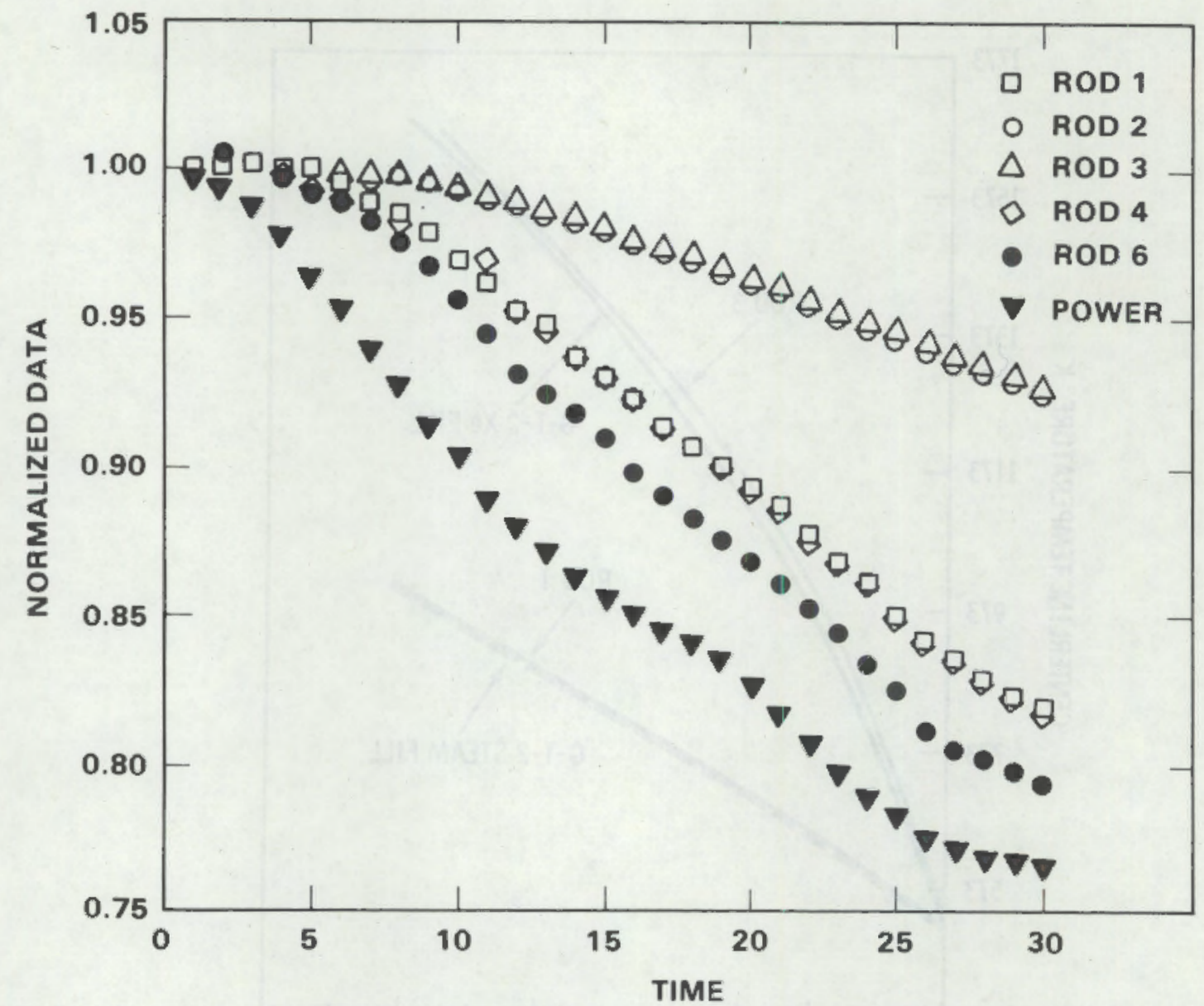

FIGURE 50. Transient Temperature Response of Representative (1ower position) Thermocouples for Run 187 (September 22, 1980)

TABLE 6. Measured and Calculated Normalized Temperature Slopes from Run 187 (a)

\begin{tabular}{|c|c|c|c|c|}
\hline $\begin{array}{l}\text { Rod } \\
\text { Number }\end{array}$ & Condition & $\begin{array}{l}\text { Temperature at } \\
17 \mathrm{~kW} / \mathrm{M} \text { (Ful1 } \\
\text { Power), K } \\
\end{array}$ & $\begin{array}{l}\text { Measured Slope } \\
\text { of Normalized } \\
\text { Temperature Versus } \\
\text { Time, } \% / \mathrm{sec}( \pm 2 \sigma) \\
\end{array}$ & $\begin{array}{l}\text { Calculated } \\
\text { Temperature Slope, } \\
\% / \sec ( \pm 2 \sigma)\end{array}$ \\
\hline $\begin{array}{l}1 \\
2 \\
3 \\
4 \\
6 \\
6\end{array}$ & $\begin{array}{l}\text { Failed } \\
\text { Resealed? } \\
\text { Unfailed } \\
\text { Failed } \\
\text { Unfailed }\end{array}$ & $\begin{array}{r}1030 \\
1675 \\
1800 \\
1000 \\
820\end{array}$ & $\begin{array}{l}0.77+0.02 \\
0.34 \mp 0.01 \\
0.34 \mp 0.01 \\
0.77 \mp 0.02 \\
0.81 \mp 0.02\end{array}$ & $\begin{array}{l}0.77+0.02 \\
0.39+0.01 \\
0.36 \mp 0.01 \\
0.78+0.02 \\
0.81+0.02\end{array}$ \\
\hline
\end{tabular}

(a) IFA-527 failed/unfailed rods, lower thermocouples.

(b) Small-gap rod. 
PRE- AND POSTFAILURE COMPARISON OF GAS PRESSURE AND CLADDING ELONGATION BEHAVIOR

The pre- and postfailure gas pressure behavior can be deduced by crosscomparison of Figures 46 and 47. For convenience, this comparison is shown for rods 1 through 5 in Figures 51 through 55. Rod 1 went to system pressure upon failure and stayed there; rod 2 suffered a pressure spike but apparently resealed and dried out; and rods 4 and 5 may have resealed but did not dry out.

The pre- and postfailure elongation behavior is shown for each rod in Figures 56 through 61 . The unfailed rods $(2,3$, and 6$)$ continued much as before except that the first startup after cold shutdown evidenced lower zero-power elongation and higher full-power elongation--perhaps due to closing axial gaps and fuel fragment release of the cladding during the cold shutdown. The erratic behavior of rod 6 elongation from September 10 to September 23 remains unexplained. The same pattern holds for the failed rods, which is surprising in view of their much reduced fuel temperatures. Apparently PCI is weak in these nominal-gap rods both before and after failure. The zero-power elongation shift establishes itself more strongly than ever after the first startup following the cold shutdown. The repeatability of this shift (only after first startups) leads to the conclusion that it is a real effect. 


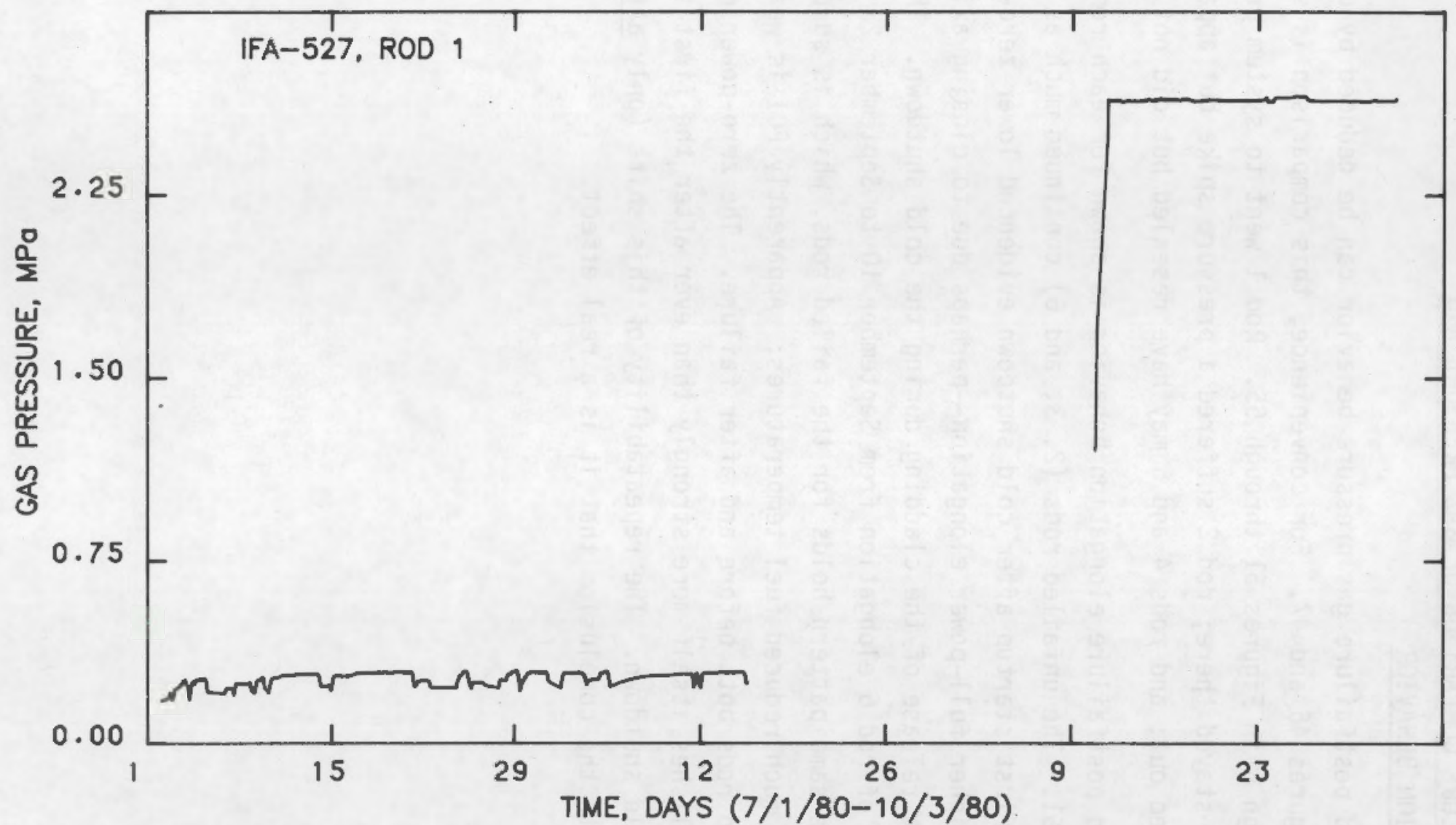

FIGURE 51. Pre- and Postfailure Gas Pressure Behavior for Rod 1 


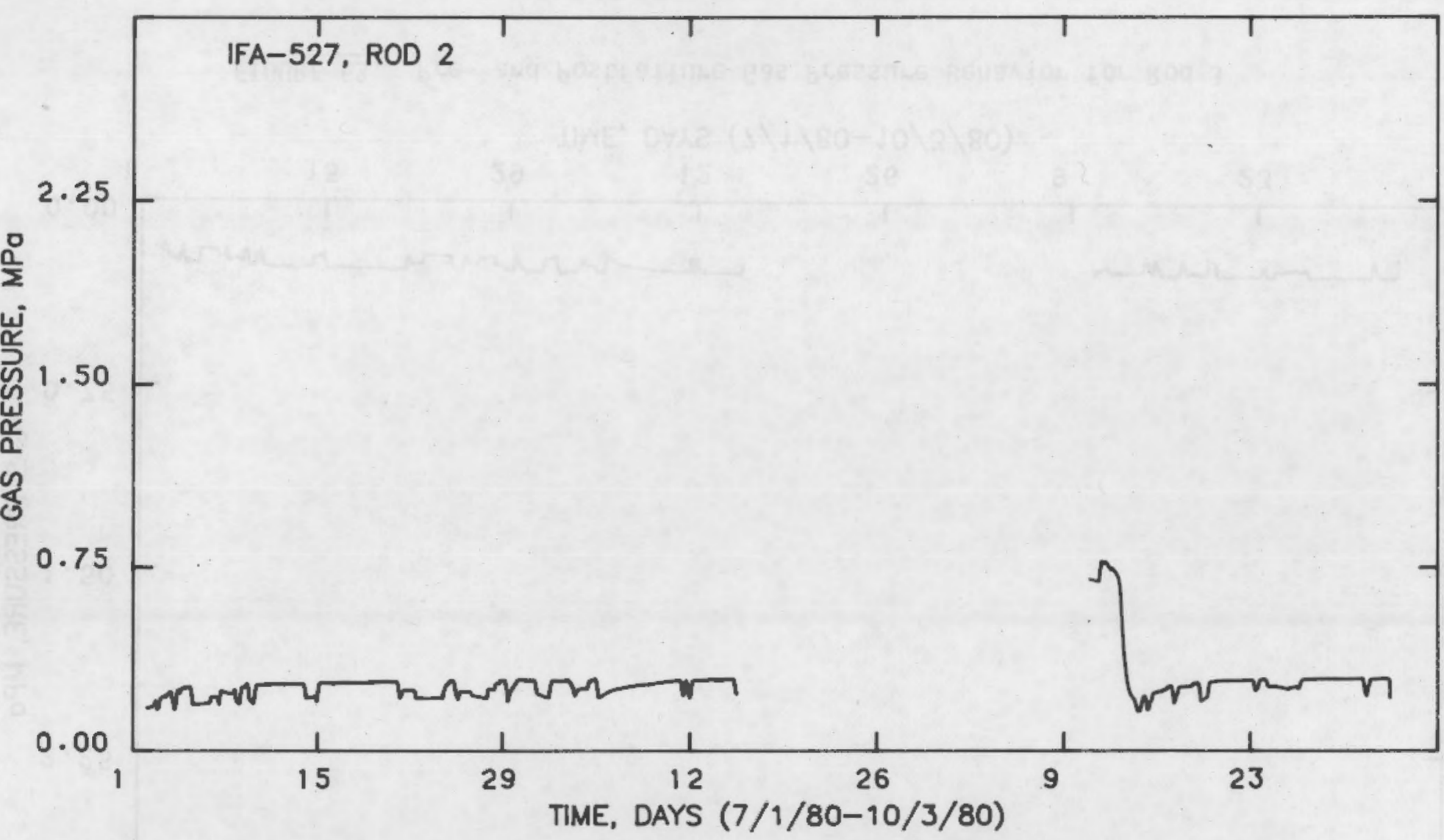

FIGURE 52. Pre- and Postfailure Gas Pressure Behavior for Rod 2 


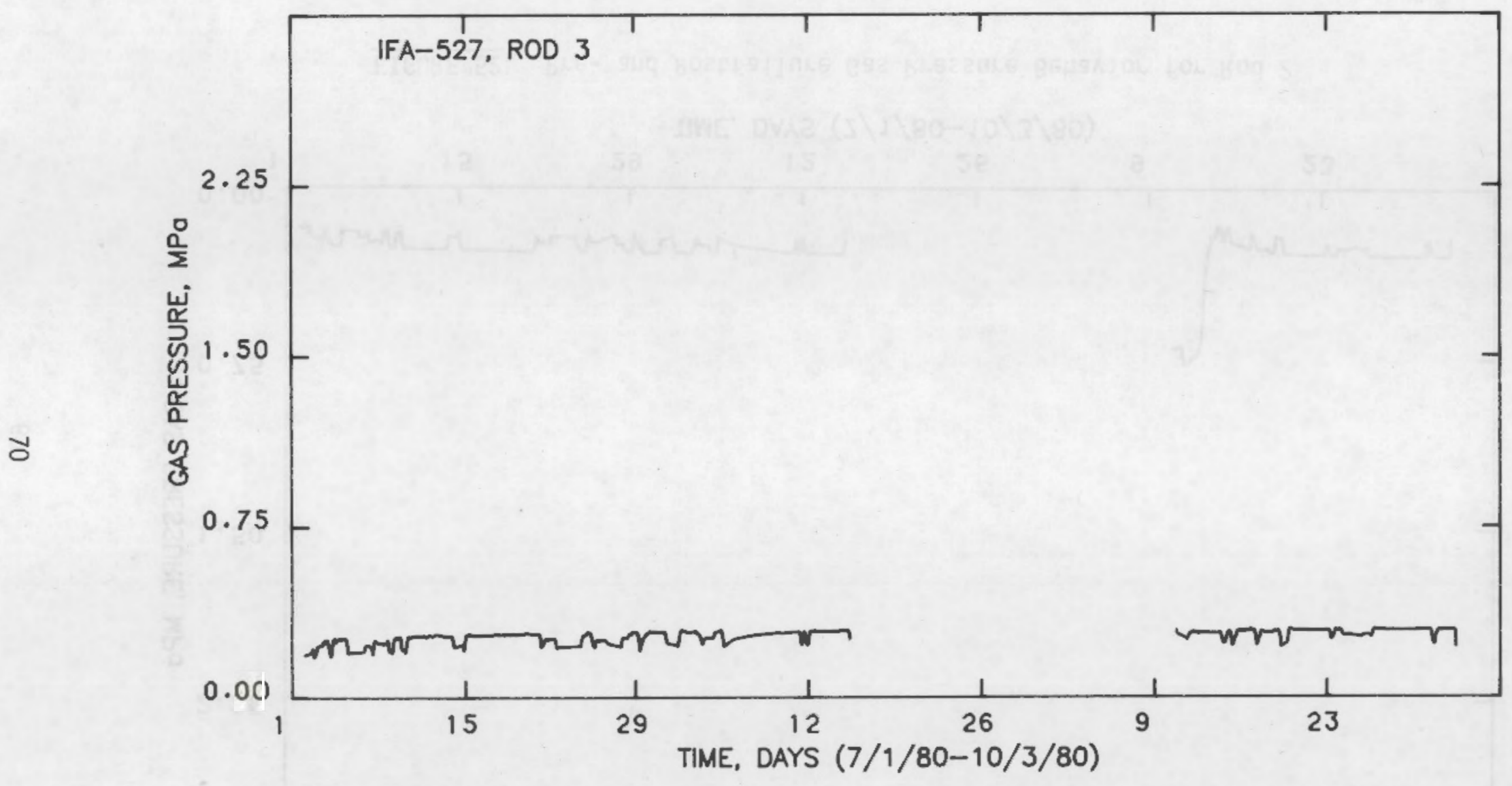

FIGURE 53. Pre- and Postfailure Gas Pressure Behavior for Rod 3 


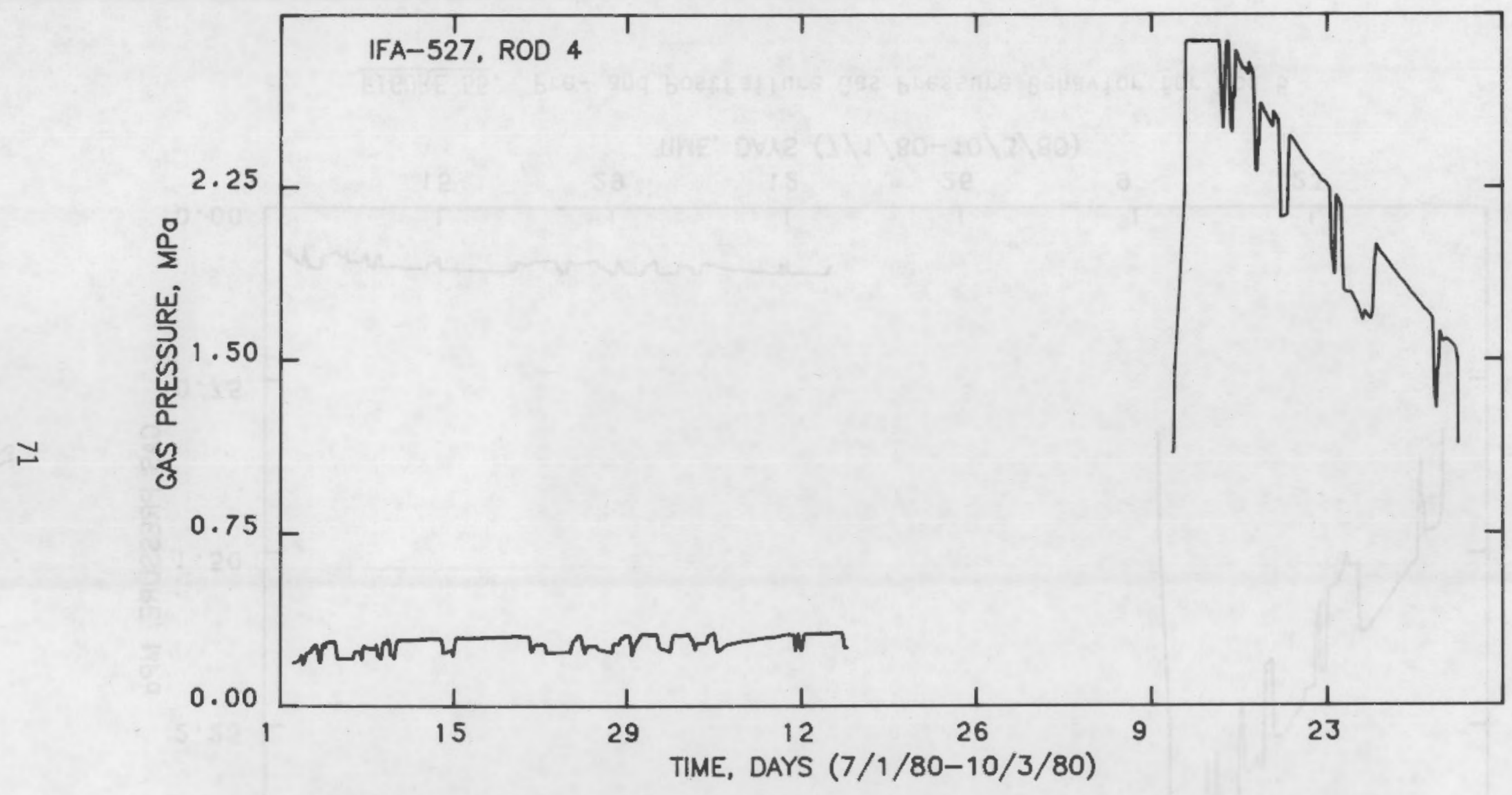

FIGURE 54. Pre- and Postfailure Gas Pressure Behavior for Rod 4 


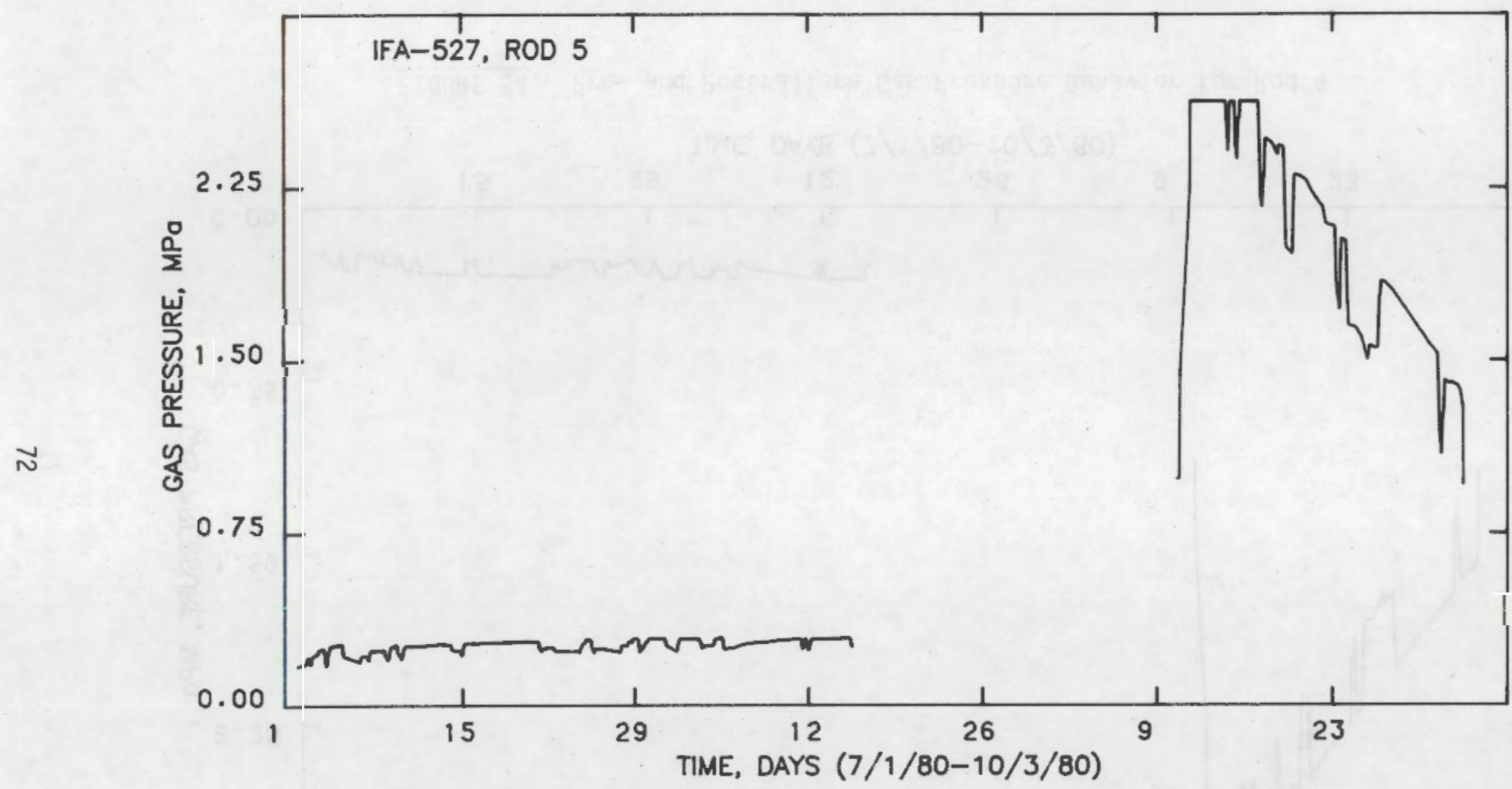

FIGURE 55. Pre- and Postfailure Gas Pressure Behavior for Rod 5 


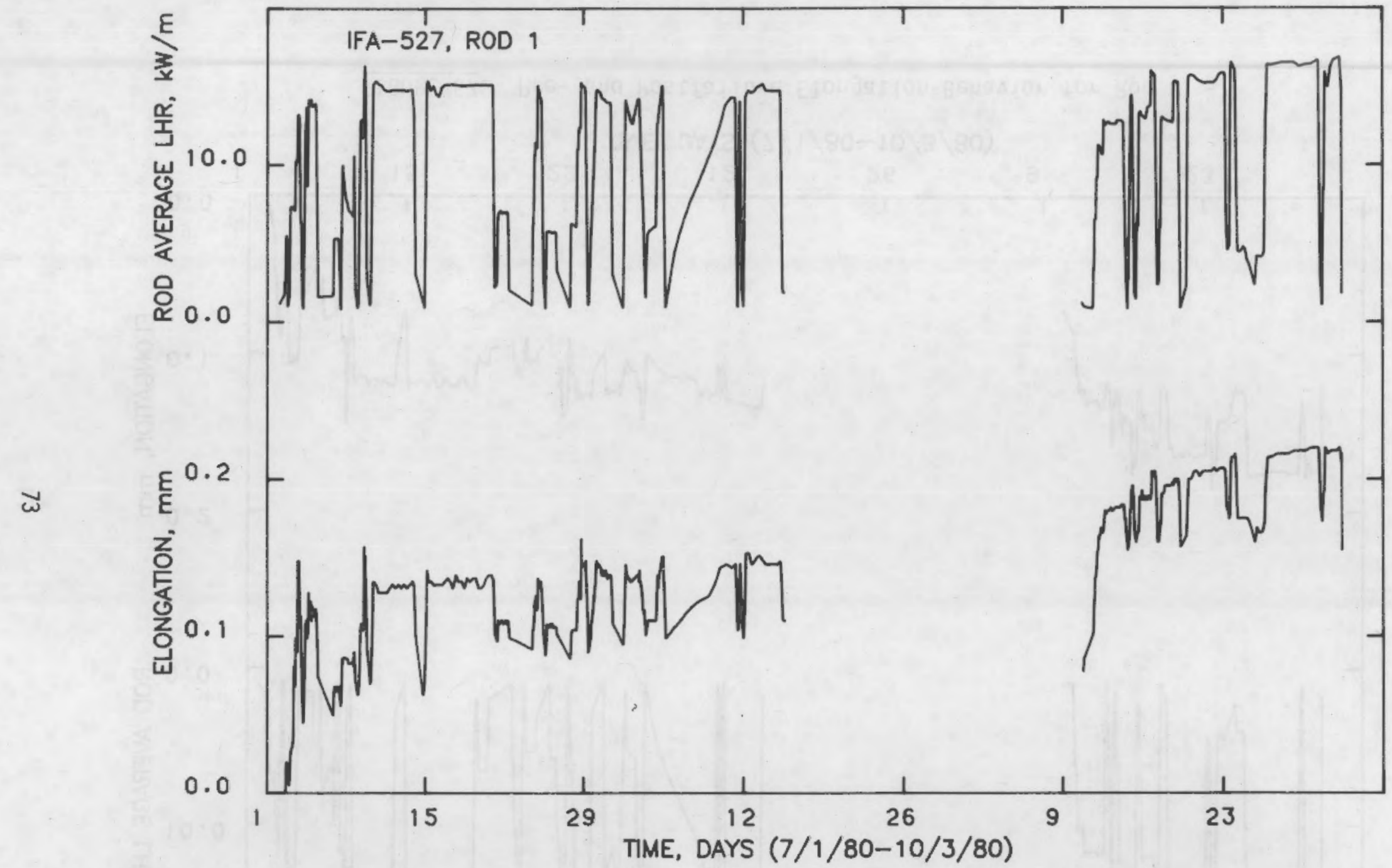

FIGURE 56. Pre- and Postfailure Elongation Behavior for Rod 1 


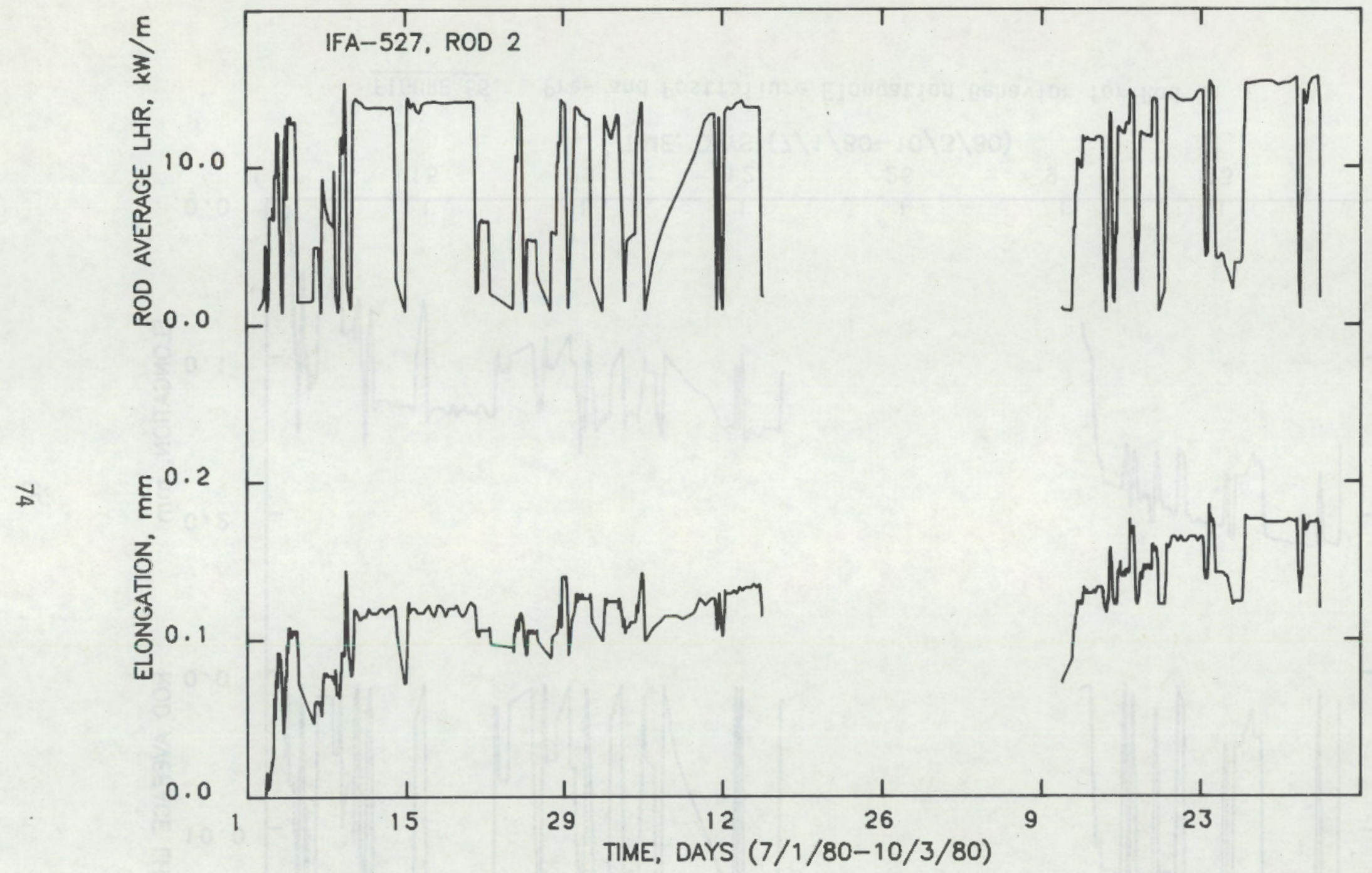

FIGURE 57. Pre- and Postfailure Elongation Behavior for Rod 2 


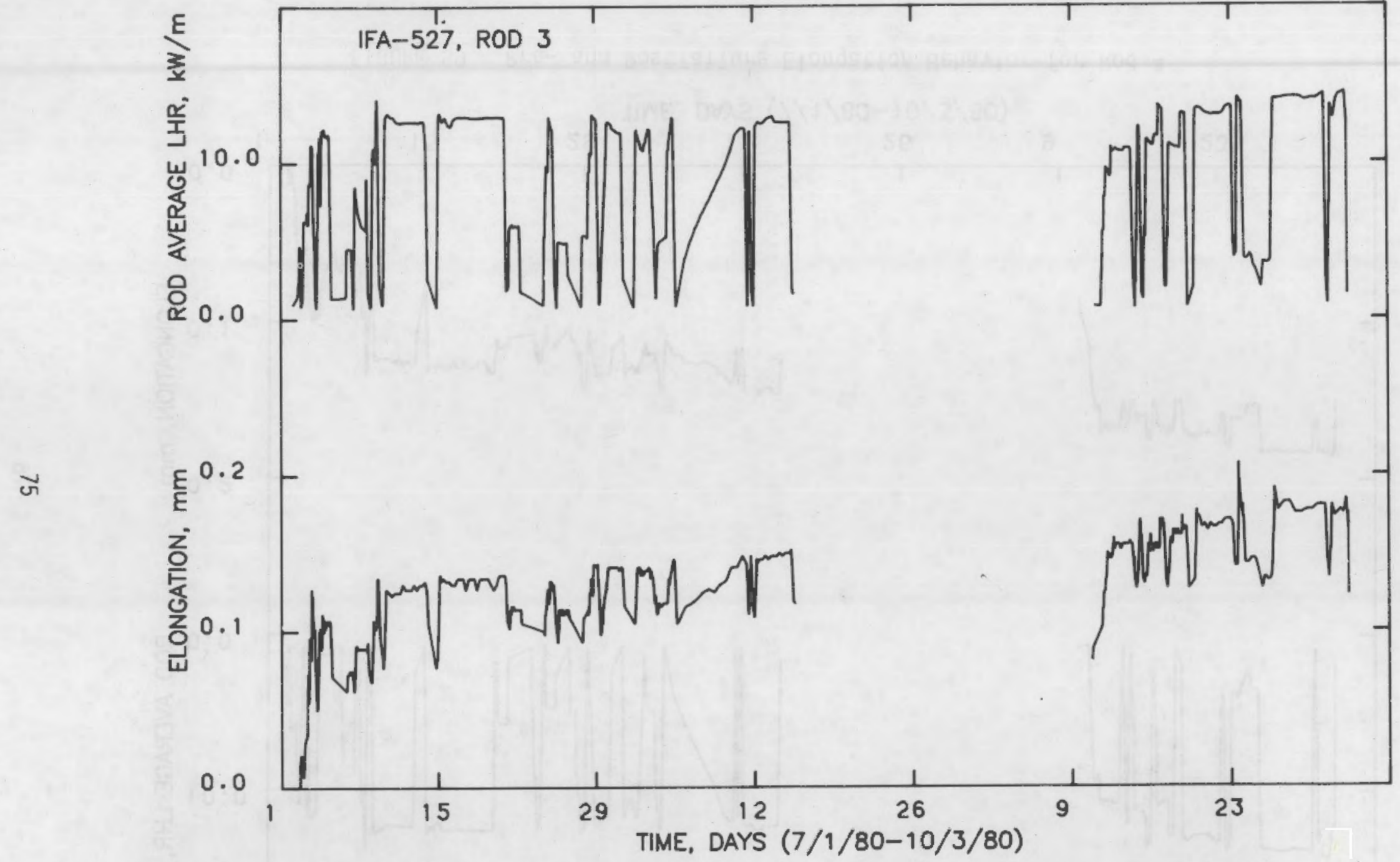

FIGURE 58. Pre- and Postfailure Elongation Behavior for Rod 3 


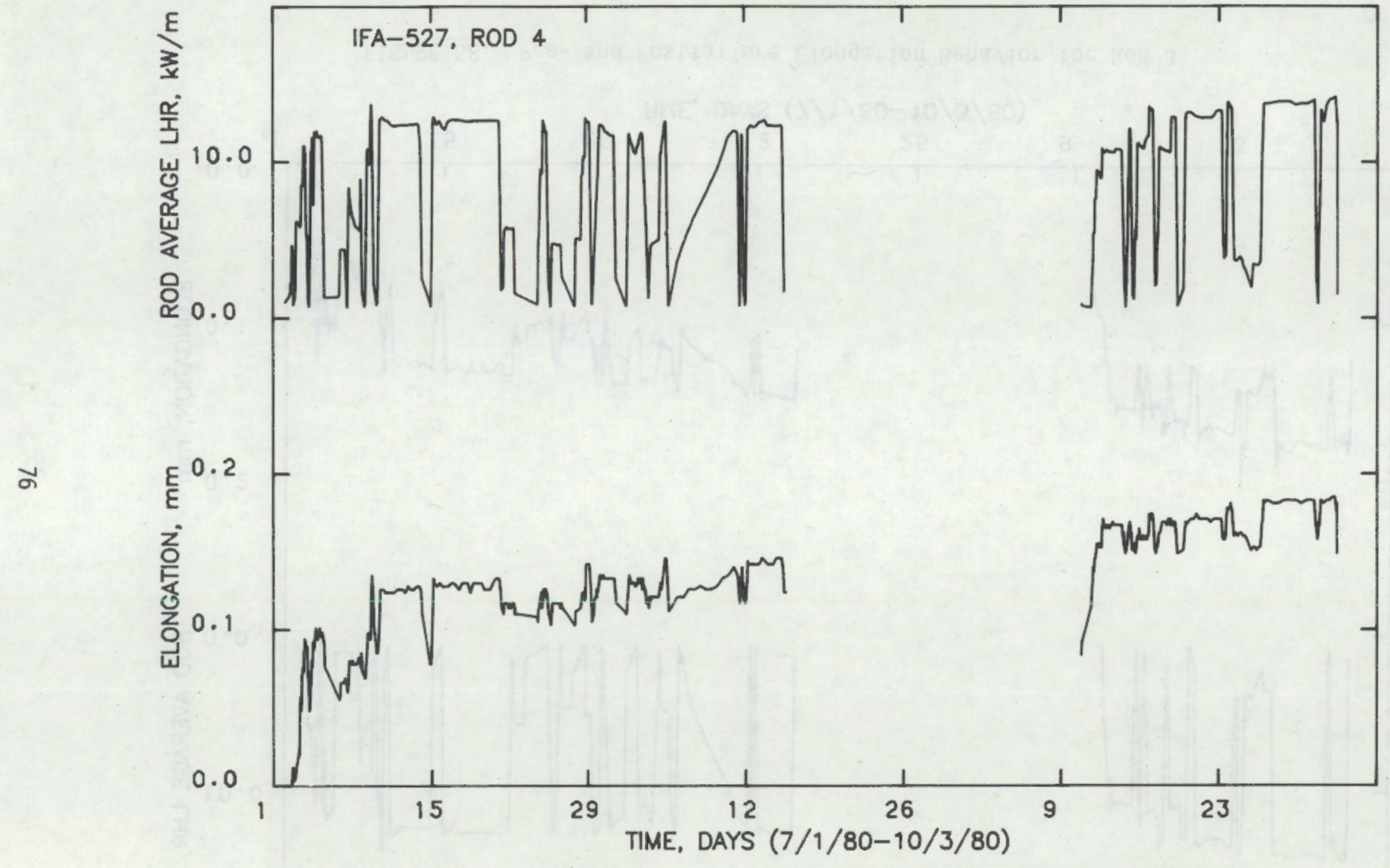

FIGURE 59. Pre- and Postfailure Elongation Behavior for Rod 4 


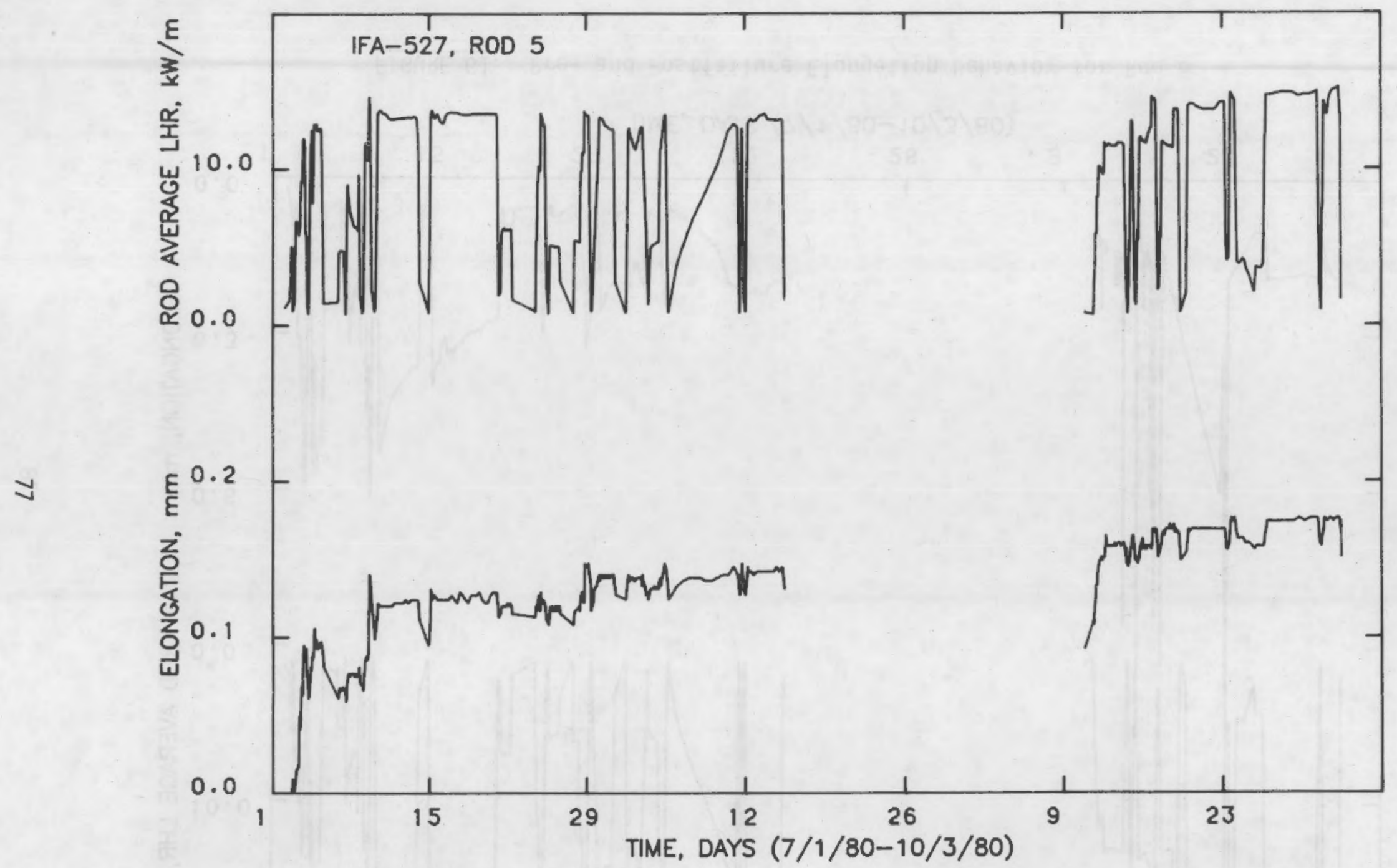

FIGURE 60. Pre- and Postfailure Elongation Behavior for Rod 5 


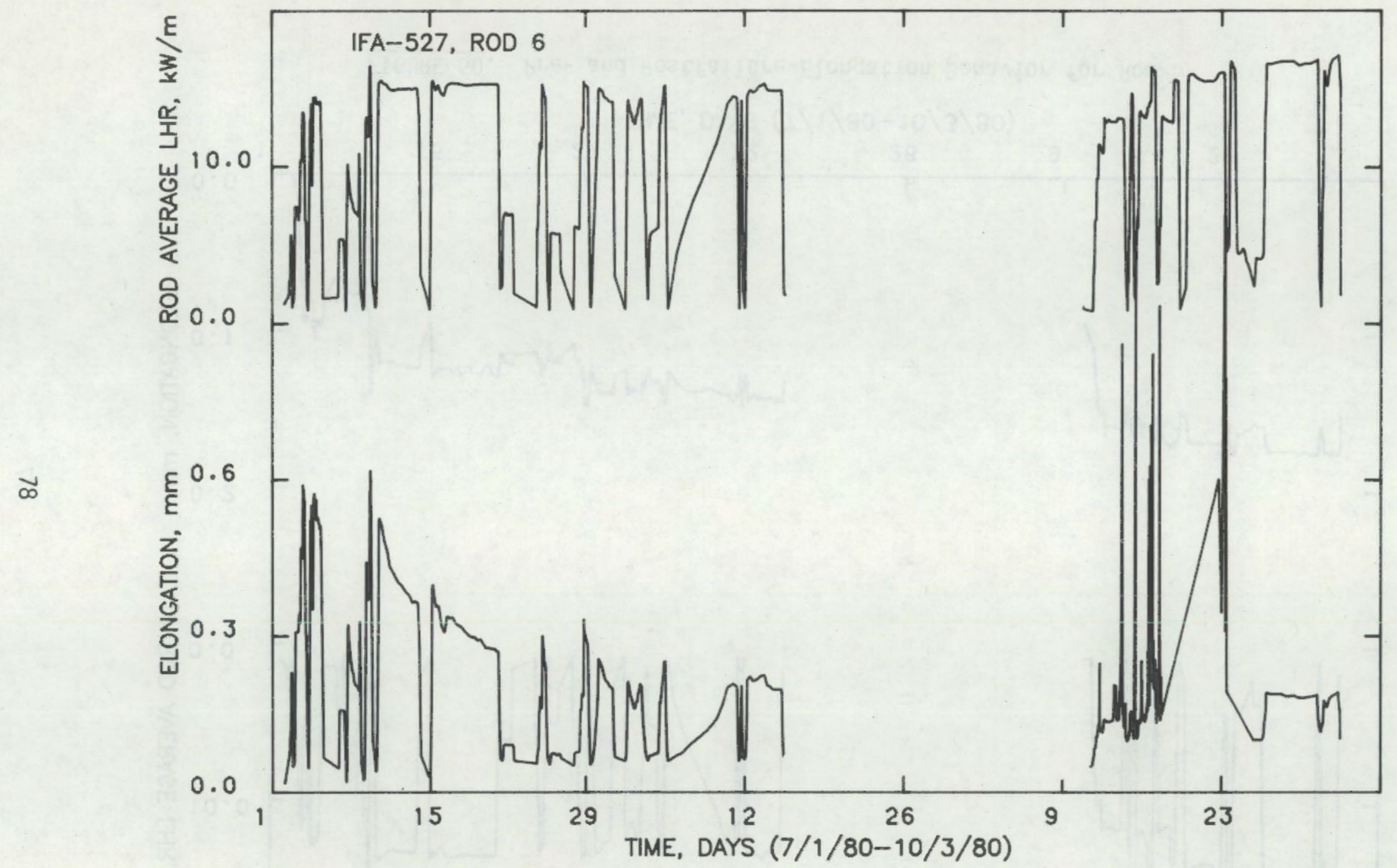

FIGURE 61. Pre- and Postfailure Elongation Behavior for Rod 6 


\section{REFERENCES}

Berna, G. A., et a1. December 1980. FRAPCON-2: A Computer Code for the Calculation of Steady-State Thermal-Mechanical Behavior of Oxide Fuel Rods. NUREG/CR-1845, U.S. Nuclear ReguTatory Commission, Washington, D.C.*

Beyer, C. E., et al. November 1975. GAPCON-THERMAL-2: A Computer Program for Calculating the Thermal Behavior of an 0xide Fuel Rod. BNWL-1898, Pacific Northwest Laboratory, Richland, Washington.

Bradley, E. R., et al. November 1979. Precharacterization Report for Instrumented Nuclear Fue? Assembly IFA-513. NUREG/CR-1077, PNL-3156, Pacific Northwest Laboratory, Richland, Washington. *

Cunningham, M. E., et al. October 1980. Application of Linear Propagation of Errors to Fuel Rod Temperature and Stored Energy Calculations. NUREG/CR-1753, PNL-3539, Pacific Northwest Laboratory, Richland, Washington. *

Cunningham, M. E., et al. May 1981. Precharacterization Report for Instrumented Fuel Assembly (IFA)-527. NUREG/CR-2168, PNL-3824, Pacific Northwest Laboratory, Richland, Washington. *

Edler, S. K., ed. November 1980. Reactor Safety Research Programs Quarterly Report, Apri1-June 198D. NUREG/CR-1454, PNL-33B0-2, Vol. 2, Pacific Northwest Laboratory, Richland, Washington. *

Hann, C. R., et al. November 1977. Test Design, Precharacterization, and Fuel Assembly Fabrication for Instrumented Fuel Assemblies IFA-431 and IFA-432. NUREG/CR-0332, BNWL-1988, Pacific Northwest Laboratory, Richland, Washington.*

Hann, C. R., et al. 1978(a). Data Report for the NRC/PNL Halden Assembly IFA-431. PNL-2494, Pacific Northwest Laboratory, Richland, Washington.

Hann, C. R., et al. 1978(b). Data Report for the NRC/PNL Haiden Assembly IFA-432. NUREG/CR-0560, PNL-2573, Pacific Northwest Laboratory, Richland, Washington. *

Lanning, D. D. November 1978. "Qualitative Confirmation of Fuel Centerline Thermocouple Data from Transient Response." American Nuclear Society Trans. 30. American Nuclear Society.

Lanning, D. D., et al. January 1979. Manifestations of Nonlinearity in Fuel Center Thermocouple Steady-State and Transient Data: Implication for Data Analysis. NUREG/CR-0220, PNL-2692, Pacific Northwest Laboratory, Richland, Washington. *

₹Available for purchase from the NRC/GPO Sales Program, U.S. Nuclear Regulatory Commission, Washington, DC 20555 and/or the National Technical Information Service, Springfield, VA 22161. 
Lanning, D. D. 1979. Use of Time-Varying Fuel Thermocouple Data to Confirm Steady-State Data Trends. Master's Thes is, University of Washington, Seattle, Washington.

Reymann, G. A., ed. February 1978. MATPR0-Version-10. TREE-NUREG-1180, EG\&G Idaho, Inc., Idaho Falls, Idaho. 


\section{DISTRIBUTION}

No. of

Copies

OFFSITE

A. A. Churm

DOE Patent Division

9300 S. Cass Avenue

Argonne, IL 60439

400 U.S. Nuclear Regulatory Commission

Division of Technical Information and Document Control

7920 Norfolk Avenue

Bethesda, MD 20014

2 DOE Technical Information Center

4 M. Silberberg

Chief, Fuel Behavior Research Branch

Division of Reactor Safety Research

U.S. Nuclear Regulatory Commission Washington, DC 20555

G. P. Marino

Fuel Behavior Research Branch

0ivision of Reactor Safety Research

U.S. Nuclear Regulatory Commission

Washington, OC 20555
No. of

Copies

50 Pacif ic Northwest Laboratory

W. J. Bailey

J. 0. Barner

E. R. 8radley

M. E. Cunningham (10)

S. K. Edler

M. D. Freshley

R. L. Goodman

R. J. Guenther

C. R. Hann

D. D. Lanning (17)

R. K. Marshall

C. L. Mohr

C. Nealliey

F. E. Panisko

W. N. Rausch

R. E. Schreiber

R. E. Williford

C. L. Wilson

Publishing Coordination Y0(2)

Technical Information (5) 



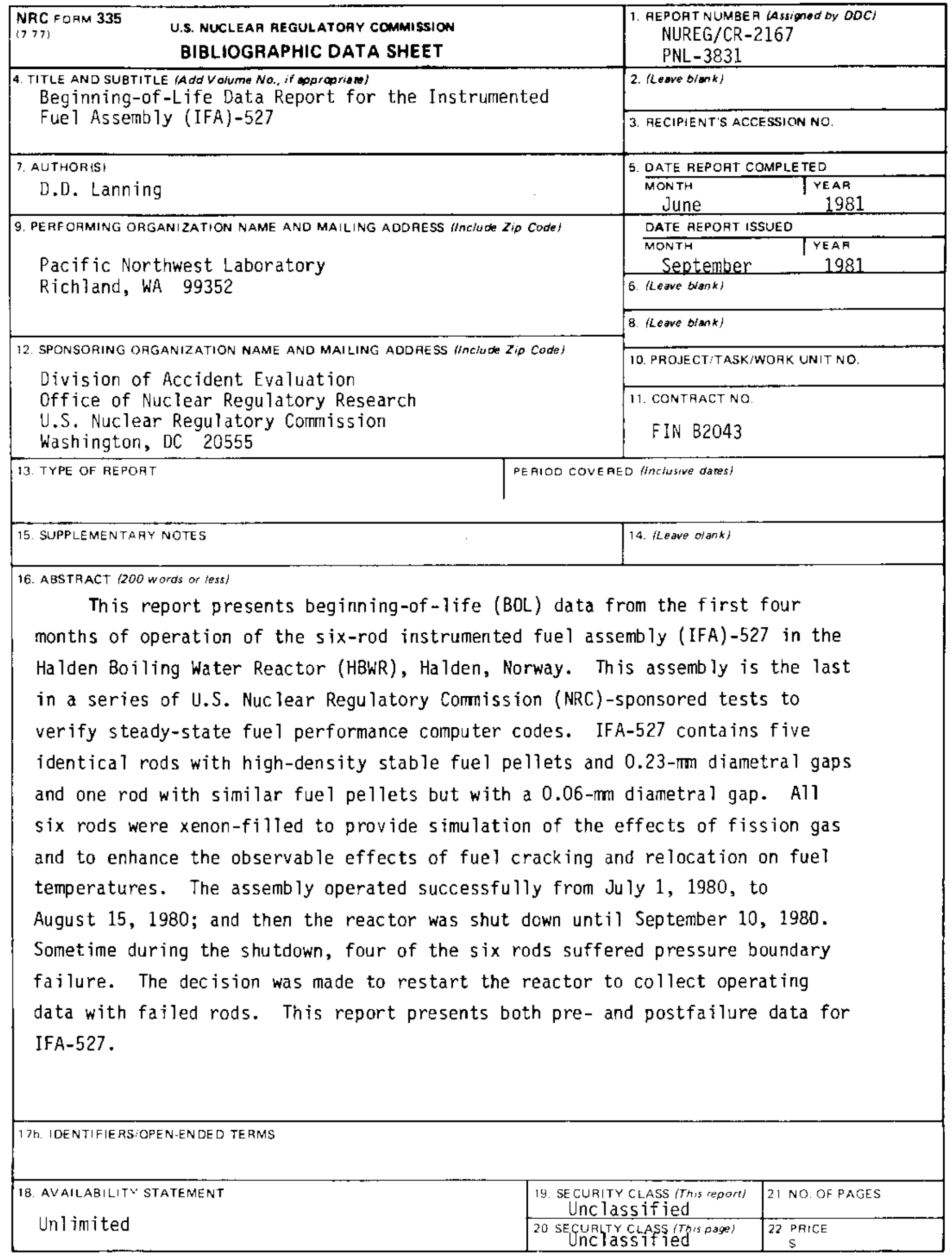


